\title{
DISSERTATION
}

The approved original version of this thesis is

\section{Explosive crystallization in thin amorphous layers on heat conducting substrates}

ausgeführt zum Zwecke der Erlangung des akademischen Grades eines

Doktors der technischen Wissenschaften unter der Leitung von

Em.O.Univ.-Prof. Dr.techn. Dr.h.c. Wilhelm Schneider E322

Institut für Strömungsmechanik und Wärmeübertragung

eingereicht an der Technischen Universität Wien

Fakultät für Maschinenwesen und Betriebswissenschaften

von

Dipl.-Ing. Christoph BUCHNER

Matr.-Nr. 0025317

Lacknergasse 108/8, A-1180 Wien

Wien, 20. Jänner 2014 


\section{Kurzfassung}

Explosive Kristallisation ist ein Transformationsprozess von einem amorphen in den kristallinen Zustand. Der selbsterhaltende Prozess wird von der Umwandlung der Schmelzenthalpie in Wärme angetrieben. In den meisten Anwendungen findet die Kristallisation in dünnen Schichten statt, die auf Substraten aufgebracht sind.

In dieser Arbeit wird ein Modell für die explosive Kristallisation in dünnen Schichten auf wärmeleitenden Substraten präsentiert. Ein bis vier Ratengleichungen beschreiben die Kinetik des Übergangs vom amorphen zum kristallinen Zustand. Für die dünne Schicht wird die Energiegleichung in einer 1D-Näherung mit einem Wärmeverlustterm gelöst. Die Wärmeleitung im Substrat wird durch eine kontinuierliche Verteilung von bewegten Wärmequellen an der Grenzfläche zur Schicht beschrieben. Es ergibt sich ein Integralausdruck für die Temperatur im Substrat in Abhängigkeit von der unbekannten Verteilung der Wärmequellen. Wenn die Temperaturleitfähigkeit des Substrates sehr klein ist gegen die Temperaturleitfähigkeit der Schicht, kann der Integralausdruck invertiert und in die Energiegleichung aufgenommen werden. Der Integralausdruck deutet auf einen nicht-lokalen Einfluss der Schichttemperatur auf den Wärmeverlust hin. Der Kontaktwärmewiderstand zwischen Schicht und Substrat kann wahlweise berücksichtigt werden.

Der gesamte Prozess wird als Welle unveränderlicher Form in einem bewegten Koordinatensystem betrachtet. Man erhält ein gekoppeltes System aus einer Integrodifferentialgleichung und ein bis vier gewöhnlichen Differentialgleichungen. Das Gleichungssystem wird numerisch mittels einer Kollokationsmethode gelöst, wobei sich die Ausbreitungsgeschwindigkeit der Welle als Eigenwert ergibt.

Typische Merkmale des Prozesses werden anhand von repräsentativen Lösungen gezeigt. Die Kristallisationszone ist sehr kurz, verglichen mit der Vorwärmzone vor der Welle. Ohne Berücksichtigung des Wärmeverlustes sind lange Kristallisationszonen und sogar unvollständige Kristallisation möglich. Wenn der Wärmeverlust berücksichtigt wird, ist die Abkühlzone hinter der Welle sehr lang im Vergleich mit der Vorwärmzone. Eine Welle unveränderlicher Form tritt nicht auf, wenn ein dimensionsloser Parameter einen bestimmten kritischen Wert überschreitet. Dieser Wert kann auch als Mindestdicke für die kristallisierende Schicht interpretiert werden.

Ausbreitungsgeschwindigkeiten der Welle werden mit Werten aus Experimenten für explosive Kristallisation von Germanium verglichen. Werte von 19 verschiedenen relevanten Parametern werden aus mehreren Quellen gesammelt. Kristallisationsparameter müssen angepasst werden, um die richtige Größenordnung der Wellenausbreitungsgeschwindigkeit zu erreichen. Für Substrattemperaturen bis ca. $700 \mathrm{~K}$ ergibt sich eine annehmbare Übereinstimmung zwischen Modell und Experiment. Für höhere Substrattemperaturen bleibt die gemessene Ausbreitungsgeschwindigkeit konstant. Dies wird von unserem Modell nicht erfasst. Bei einer Substrattemperatur von ca. $775 \mathrm{~K}$ ist die gemessene Ausbreitungsgeschwindigkeit in Übereinstimmung mit unserem Modell nahezu unabhängig von der Schichtdicke. Mögliche Quellen für die Diskrepanzen zwischen Modell und Experiment werden identifiziert, und mögliche weitere Bereiche für Verbesserungen werden diskutiert. 


\section{Abstract}

Driven by the liberation of the latent heat of fusion, a transformation from an amorphous state to the crystalline state may take place in a progressing wave. The process is self-sustaining and is often called "explosive crystallization". In most applications, the crystallization process takes place in thin layers that are mounted on a substrate.

In this work, a model for explosive crystallization in a thin amorphous layer on a heat conducting substrate is presented. One to four rate equations are used to describe the kinetics of the amorphous-crystalline transition. For the thin layer, the energy equation is used in a one-dimensional approximation with a heat-loss term. Heat conduction in the substrate is described by introducing a continuous distribution of moving heat sources at the interface. This gives an integral representation for the temperature in the substrate in terms of the unknown source distribution. Provided the substrate's thermal diffusivity is much smaller than the thermal diffusivity of the layer, the integral representation can be inverted and included in the energy equation of the layer. The integral term implies that there is a non-local influence of the temperature distribution in the layer on the heat loss. Optionally, a thermal contact resistance at the interface between layer and substrate is taken into account.

The whole process is examined as a wave of invariant shape in a moving frame of reference. A coupled system of one integro-differential equation and one to four ordinary differential equations is obtained. It is solved numerically using a collocation method. The propagation velocity of the wave is obtained as an eigenvalue of the system of equations.

Some representative solutions of the system of equations are shown, demonstrating the key features of the process: Typically, the crystallization zone is short compared to the thermal preheating zone in front of the wave. Long crystallization zones and even incomplete crystallization are possible in cases without heat loss. When heat loss is taken into account, the cooling zone behind the wave is long compared to the pre-heating zone. Varying a non-dimensional heat loss parameter, a critical value is found beyond which no crystallization wave of invariant shape is possible. This can also be interpreted as a certain minimum layer thickness.

Finally, crystallization-wave velocities are compared with experimental values for explosive crystallization in germanium. Data for 19 different experimental and material parameters are collated from a number of sources. It is necessary to adjust crystallization parameters to achieve a correct magnitude of the wave propagation velocity. For substrate temperatures up to about $700 \mathrm{~K}$, the agreement between the analysis and experimental values is reasonable. For larger substrate temperatures, the wave velocity in the experiment remains approximately constant, which is not reflected in the model results. Furthermore, in the experiment, the wave velocity is nearly independent of the layer thickness at a substrate temperature of approximately $775 \mathrm{~K}$. This is in accord with our model. Possible sources for the discrepancies between the experimental results and the model are identified and potential areas for future work are discussed. 


\section{Acknowledgements}

First and foremost, I would like to express my gratitude towards my advisor Prof. Wilhelm Schneider. His guidance was very valuable, and he was always available for fruitful and instructive discussions. He greatly expanded my knowledge about the scientific method of working, effective problem analysis, and how to successfully conduct fundamental research.

I would also like to express my thanks to Prof. Alfred Kluwick, who kindly agreed to become the second advisor.

Furthermore, I would like to thank the members of the Institute of Fluid Mechanics and Heat Transfer, whose knowledge and input proved very valuable. Prof. Herbert Steinrück and Dr. Thomas Loimer always had an open ear for my questions. DI Ernst Hofmann, Dr. Richard Jurisits, Dr. Georg Meyer, DI Markus Müllner, DI Thomas Müllner, Dr. Ulrich Schoisswohl and DI Johannes Strecha receive my thanks for many fruitful lunchtime discussions.

Financial support by Androsch International Management Consulting GmbH (AIC) in the course of preparing this work is gratefully acknowledged.

Finally, I would like to thank my partner Birgit, whose unfailing support was invaluable during my work on this thesis. 


\section{Contents}

$\begin{array}{ll}\text { Nomenclature } & 1\end{array}$

1. Introduction $\mathbf{5}$

1.1. The explosive crystallization phenomenon . . . . . . . . . . 5

1.2. Heat loss influence . . . . . . . . . . . . . . . . 5

1.3. Materials . . . . . . . . . . . . . . . . 6

1.4. Applications . . . . . . . . . . . . . . . . 6

1.5. Motivation of present work . . . . . . . . . . . . 7

\begin{tabular}{ll}
\hline 2. Problem formulation & 8
\end{tabular}

3. Basic equations 10

3.1. Energy equation . . . . . . . . . . . . . . . . . . . . . . 10

3.2. Rate equations . . . . . . . . . . . . . . . . . . . . . . 11

3.2.1. Rate equations for heterogeneous crystallization . . . . . . . . 12

3.2.2. Simplification to one rate equation . . . . . . . . . . . . . 12

3.2.3. Rate equations for homogeneous crystallization . . . . . . . . 13

3.3. Crystal growth velocity . . . . . . . . . . . . . . . . . . 14

3.4. Crystal nucleation rate . . . . . . . . . . . . . . . 16

3.5. Crystallization time . . . . . . . . . . . . . . . . . . 17

\begin{tabular}{lr}
\hline 4. Non-dimensional formulation & 19
\end{tabular}

4.1. Non-dimensional variables . . . . . . . . . . . . . . . . . . . . . . . . 19

4.2. Classification of crystallization waves . . . . . . . . . . . . . . 22

4.3. Non-dimensional formulation of the crystal growth velocity . . . . . . 22

4.4. Non-dimensional formulation of the crystal nucleation rate . . . . . . 23

4.5. Crystallization wave of invariant shape . . . . . . . . . . . . . . 25

4.5.1. Energy equation and non-dimensional parameters . . . . . . . 25

4.5.2. Heterogeneous crystallization . . . . . . . . . . . 26 
4.5.3. Single rate equation $\ldots \ldots \ldots \ldots \ldots \ldots$

4.5.4. Homogeneous crystallization. . . . . . . . . . . . . . . 26

5. Treatment of substrate 28

5.1. Integral representation of the temperature in the substrate . . . . 28

5.2. Expansion for weakly conducting substrates . . . . . . . . . . . 29

5.2.1. Expansion of the integral representation for small values of a. 29

5.2 .2 . Inversion of the interface coupling condition . . . . . . . . 31

$5.2 .3 . \quad$ Elimination of $\dot{q}$ in the energy equation $\ldots \ldots \ldots . . . .32$

5.3. Thermal contact resistance at the interface . . . . . . . . . . . . 32

5.3.1. Expansion for small thermal contact resistance R . . . . . . 33

5.3.2. Expansion for large thermal contact resistance R $\ldots \ldots . .35$

\begin{tabular}{ll}
\hline 6. & Precursor region \\
\hline 7
\end{tabular}

6.1. Temperature distribution for $\mathrm{R}=0 \ldots \ldots \ldots \ldots \ldots \ldots$

6.2. Heat loss distribution for $\mathrm{R}=0$. . . . . . . . . . . . . . . . . 38

6.3. Influence of thermal contact resistance . . . . . . . . . . . 39

6.3.1. Small values of $\mathrm{R} \ldots \ldots \ldots$

6.3.2. Large values of $\mathrm{R} \ldots \ldots \ldots \ldots$

$\begin{array}{ll}\text { 7. Asymptotic behaviour far behind the wave } & 41\end{array}$

7.1. Case of negligible thermal contact resistance, $\mathrm{R}=0$. . . . . . . 41

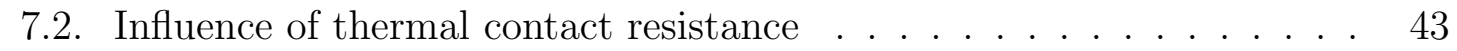

7.2.1. Small values of R . . . . . . . . . . . . . . . . 43

$7.2 .2 . \quad$ Large values of $\mathrm{R} \ldots \ldots \ldots \ldots \ldots$

8. Stretched coordinate system 44

8.1. Coordinate transformation . . . . . . . . . . . . . . . . 44

8.2. Transformed system of equations . . . . . . . . . . . . 44

8.2 .1 . Energy equation for $\mathrm{R}=0 \ldots \ldots \ldots \ldots \ldots \ldots$

8.2 .2 . Approximation for small values of $\mathrm{R} \ldots \ldots \ldots \ldots$

8.2 .3 . Approximation for large values of $\mathrm{R} \ldots \ldots \ldots \ldots$

8.2.4. Rate equations . . . . . . . . . . . . . . . 46

9. Numerical method of solution 47

9.1. Overview of solution procedure $\ldots \ldots \ldots \ldots \ldots 47$

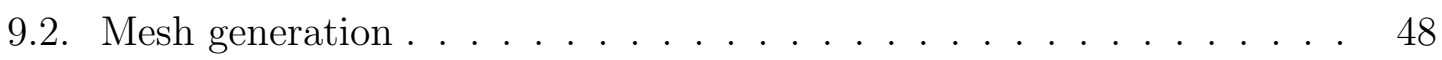


9.3. Iteration procedure $\ldots \ldots \ldots \ldots \ldots \ldots \ldots$

9.3.1. Treatment of the integral term . . . . . . . . . . . . 49

9.3.2. Iteration methods for integral term . . . . . . . . . . 50

9.4. Asymptotic boundary condition near $\mathrm{z}=1$. . . . . . . . . . . 50

9.5. Computation of the heat loss term $\ldots \ldots \ldots \ldots \ldots \ldots$

10.Parameter determination 54

10.1. Germanium . . . . . . . . . . . . . . . . . 54

10.1.1. Parameter sets . . . . . . . . . . . . . . 54

10.1.2. Maximum crystalline volume fraction . . . . . . . . . . 55

10.1.3. Mass density . . . . . . . . . . . . . . . 55

10.1.4. Glass transition temperature . . . . . . . . . . 55

10.1.5. Melting temperature . . . . . . . . . . . . 56

10.1.6. Latent heat of melting . . . . . . . . . . . . 56

10.1.7. Isobaric specific heat capacity . . . . . . . . . . . . 57

10.1.8. Thermal diffusivity . . . . . . . . . . . . . . 57

10.1.9. Thermal conductivity . . . . . . . . . . . 57

10.1.10. Crystal growth velocity . . . . . . . . . . . 58

10.1.11. Crystal nucleation rate . . . . . . . . . . . . . . . . 59

10.1.12.Characteristic crystallization time. . . . . . . . . . 60

10.2. Substrate materials . . . . . . . . . . . . . . . . . 60

10.3. Parameters in experiments . . . . . . . . . . . . . 61

10.3.1. Film thickness . . . . . . . . . . . . . . . . . . 61

10.3.2. Substrate temperature . . . . . . . . . . . . . . 62

10.3.3. Thermal contact resistance . . . . . . . . . . . . . . 62

10.3.4. Wave propagation velocity . . . . . . . . . . . . . . . 62

$\begin{array}{ll}\text { 11. Results } & 63\end{array}$

11.1. Illustrative solutions $\ldots \ldots \ldots \ldots$

11.2. Variation of the specific latent heat of melting . . . . . . . . . 65

11.2.1. Adiabatic case . . . . . . . . . . . . . . 65

11.2.2. Case including heat loss $\ldots \ldots \ldots \ldots \ldots \ldots$

11.2.3. Plateau region of nearly constant temperature . . . . . . . 67

11.2.4. Non-local influence on heat loss . . . . . . . . . . . . . 69

11.3. Variation of heat loss parameter $\mathrm{H}$

11.3.1. Critical value of $\mathrm{H} \ldots \ldots \ldots \ldots$ 
11.3.2. Plateau solutions . . . . . . . . . . . . . . . . . 72

11.3.3. Effective criticality . . . . . . . . . . . . . . 74

11.4. Variation of thermal contact resistance . . . . . . . . . . . 74

11.5. Length of crystallization zone . . . . . . . . . . . . . 76

11.6. Comparison with experiments . . . . . . . . . . . 77

11.6.1. Kinetic prefactor . . . . . . . . . . . . . . 78

11.6.2. Effect of stopping crystallization below a certain temperature 79

11.6.3. Results for different layer thickness . . . . . . . . . . . 81

11.6.4. Potential sources of discrepancies . . . . . . . . . . . . 82

11.7. Future work . . . . . . . . . . . . . . . . . . . . . 83

\begin{tabular}{ll}
\hline 12. Summary & 85
\end{tabular}

\begin{tabular}{ll}
\hline A. Appendix & $\mathbf{8 8}$
\end{tabular}

A.1. Derivation of rate equations for homogeneous crystallization . . . . 88

\begin{tabular}{ll}
\hline Bibliography & 91
\end{tabular} 


\section{Nomenclature}

See Eq.

\section{Latin Letters}

\begin{tabular}{|c|c|c|}
\hline$a$ & $\begin{array}{l}\text { ratio of thermal diffusivities of substrate } \\
\text { and layer }\end{array}$ & - \\
\hline$C_{1}-C_{4}$ & crystal growth velocity parameters & - \\
\hline$C_{1, G}, C_{2, G}$ & crystal growth velocity parameters & - \\
\hline$C_{1, I}, C_{2, I}$ & crystal nucleation rate parameters & - \\
\hline$C_{\infty}$ & pre-factor of $\Theta(\eta)$ far behind the wave & - \\
\hline$c_{p}$ & isobaric specific heat capacity & $\mathrm{J} / \mathrm{kg} \mathrm{K}$ \\
\hline$E$ & auxiliary activation energy & $\mathrm{eV}$ \\
\hline$E_{1}, E_{2}$ & kinetic constants & $\mathrm{K}$ \\
\hline$E_{G}, E_{G m}$ & activation energies for $G_{C}$ & $\mathrm{eV}$ \\
\hline$E_{I}, E_{I m}$ & activation energies for $I_{C}$ & $\mathrm{eV}$ \\
\hline$E_{\tau}$ & activation energy for $\tau$ & $\mathrm{eV}$ \\
\hline$e$ & auxiliary abbreviation & $\mathrm{W} \mathrm{s}^{1 / 2} / \mathrm{m}^{2} \mathrm{~K}$ \\
\hline$f, f_{0}$ & auxiliary quantities & $\mathrm{m} / \mathrm{s}$ \\
\hline$G$ & non-dimensional crystal growth velocity & - \\
\hline$G_{0}$ & crystal growth velocity prefactor & $\mathrm{m} / \mathrm{s}$ \\
\hline$G_{C}$ & crystal growth velocity & $\mathrm{m} / \mathrm{s}$ \\
\hline$G_{C, r e f}$ & reference crystal growth velocity & $\mathrm{m} / \mathrm{s}$ \\
\hline$g$ & auxiliary function & - \\
\hline$H$ & non-dimensional heat loss parameter & - \\
\hline$I$ & non-dimensional crystal nucleation rate & - \\
\hline$I_{0}$ & crystal nucleation rate prefactor & $1 / \mathrm{s} \mathrm{m}^{3}$ \\
\hline$I_{C}$ & crystal nucleation rate & $1 / \mathrm{s} \mathrm{m}^{3}$ \\
\hline$I_{C, r e f}$ & reference crystal nucleation rate & $1 / \mathrm{s} \mathrm{m}^{3}$ \\
\hline
\end{tabular}




\begin{tabular}{|c|c|c|c|}
\hline Symbol & Quantity & Units & See Eq. \\
\hline$K$ & constant for temperature precursor & - & $(6.3)$ \\
\hline$k$ & thermal conductivity & $\mathrm{W} / \mathrm{m} \mathrm{K}$ & $(3.1)$ \\
\hline $\mathrm{K}_{0}$ & modified Bessel function of the second kind & - & $(5.1)$ \\
\hline$k_{B}$ & Boltzmann constant & $\mathrm{eV} / \mathrm{K}$ & $(3.16)$ \\
\hline$L$ & perturbation function & - & $(9.1)$ \\
\hline$l$ & specific latent heat & $\mathrm{J} / \mathrm{kg}$ & $(3.1)$ \\
\hline$L_{C}$ & average distance between crystal nuclei & $\mathrm{m}$ & $(3.7)$ \\
\hline$L_{r e f}$ & reference length for heat conduction & $\mathrm{m}$ & $(4.5)$ \\
\hline$M$ & non-dimensional crystallization parameter & - & $(4.35)$ \\
\hline$m$ & dimensionality of crystal growth & - & $(3.5)$ \\
\hline $\bar{N}$ & initial number of nuclei per unit volume & $1 / \mathrm{m}^{3}$ & $(3.7)$ \\
\hline$N$ & auxiliary function & - & $(6.4)$ \\
\hline$n$ & $\begin{array}{l}\text { non-dimensional activation probability of } \\
\text { crystal nuclei }\end{array}$ & - & $(4.17)$ \\
\hline$n_{C}$ & activation probability of crystal nuclei & $1 / \mathrm{s}$ & $(3.5)$ \\
\hline$n_{C, r e f}$ & $\begin{array}{l}\text { reference activation probability of crystal } \\
\text { nuclei }\end{array}$ & $1 / \mathrm{s}$ & $(4.13)$ \\
\hline$P$ & kinetic prefactor & - & $(11.6)$ \\
\hline$P_{1}, P_{2}$ & constant auxiliary parameters & - & $(11.2)$ \\
\hline$\dot{q}$ & non-dimensional interface heat flux & - & $(4.15)$ \\
\hline$\dot{q}_{i}$ & interface heat flux & $\mathrm{W} / \mathrm{m}^{2}$ & $(3.1)$ \\
\hline$\dot{q}_{r e f}$ & reference heat flux & $\mathrm{W} / \mathrm{m}^{2}$ & $(4.6)$ \\
\hline$R$ & dimensionless thermal contact resistance & - & $(5.18)$ \\
\hline$R_{i}$ & thermal contact resistance & $\mathrm{m}^{2} \mathrm{~K} / \mathrm{W}$ & $(5.16)$ \\
\hline$R^{*}$ & scaled $R$ & - & $(5.32)$ \\
\hline$S_{1}, S_{2}, S_{3}$ & constant auxiliary parameters & - & $(11.4)$ \\
\hline$T$ & absolute temperature & $\mathrm{K}$ & $(3.1)$ \\
\hline$t$ & time & $\mathrm{s}$ & $(3.1)$ \\
\hline$t_{C}$ & characteristic time of crystallization & $\mathrm{s}$ & $(4.8)$ \\
\hline$t_{C, r e f}$ & ref. characteristic time of crystallization & s & $(4.11)$ \\
\hline$T^{*}$ & temperature of maximum $G_{C}$ & $\mathrm{~K}$ & $(3.20)$ \\
\hline$T_{\text {ref }}$ & reference temperature & $\mathrm{K}$ & $(4.9)$ \\
\hline$T_{t o t}$ & total end temperature & $\mathrm{K}$ & $(4.1)$ \\
\hline$U$ & wave propagation velocity & $\mathrm{m} / \mathrm{s}$ & $(4.5)$ \\
\hline
\end{tabular}




\begin{tabular}{clcc} 
Symbol & Quantity & Units & See Eq. \\
\hline$V$ & volume fraction of crystalline phase & - & $(2.1)$ \\
$V_{\infty}$ & max. volume fraction of crystalline phase & - & $(2.1)$ \\
$x$ & cartesian coordinate & $\mathrm{m}$ & $(3.1)$ \\
$Y$ & non-dimensional cartesian coordinate & - & $(5.1)$ \\
$y$ & cartesian coordinate & $\mathrm{m}$ & $(3.1)$ \\
$z$ & stretched non-dimensional wave coordi- & - & $(8.1)$
\end{tabular}

\section{Greek Letters}

\begin{tabular}{|c|c|c|}
\hline$\alpha$ & thermal diffusivity & $\mathrm{m}^{2} / \mathrm{s}$ \\
\hline$\beta$ & coordinate stretching parameter & - \\
\hline$\delta_{L}$ & layer thickness & $\mathrm{m}$ \\
\hline$\eta$ & non-dimensional wave coordinate & - \\
\hline$\eta_{B}$ & auxiliary value of $\eta$ & - \\
\hline$\eta_{C}$ & wave coordinate where (7.1) becomes valid & - \\
\hline$\Theta$ & non-dimensional temperature difference & - \\
\hline$\lambda$ & non-dimensional wave eigenvalue & - \\
\hline$\mu$ & non-dimensional crystallization parameter & - \\
\hline$\xi$ & degree of crystallization & - \\
\hline$\rho$ & mass density & $\mathrm{kg} / \mathrm{m}^{3}$ \\
\hline$\sigma$ & crystal growth shape coefficient & - \\
\hline$\varsigma$ & integration variable & - \\
\hline$\tau$ & crystallization time & $\mathrm{s}$ \\
\hline$\tau_{0}$ & crystallization time prefactor & $\mathrm{s}$ \\
\hline$\phi_{i=0 . . m}$ & $\begin{array}{l}\text { non-dimensional rate equation quantities, } \\
\text { homogeneous crystallization }\end{array}$ & - \\
\hline$\Phi$ & $\begin{array}{l}\text { function of degree of heterogeneous crys- } \\
\text { tallization }\end{array}$ & - \\
\hline$\varphi_{i=0 . . m}$ & $\begin{array}{l}\text { rate equation quantities, heterogeneous } \\
\text { crystallization }\end{array}$ & - \\
\hline$\phi_{C, i=0 . . m}$ & $\begin{array}{l}\text { rate equation quantities, homogeneous } \\
\text { crystallization }\end{array}$ & $\mathrm{m}^{-\mathrm{i}}$ \\
\hline$\Omega$ & auxiliary function & - \\
\hline
\end{tabular}


Superscripts

functions in $z$ coordinate space

Subscripts

$\begin{array}{cl}a & \text { amorphous } \\ a d & \text { adiabatic } \\ C & \text { crystal, crystallization } \\ c & \text { crystalline } \\ g & \text { glass transition } \\ L & \text { layer } \\ \text { max } & \text { maximum } \\ m & \text { melt, melting } \\ r e f & \text { reference quantity } \\ S & \text { substrate }\end{array}$




\section{Introduction}

\subsection{The explosive crystallization phenomenon}

Explosive crystallization is a self-sustaining process of transformation of a solid material from an amorphous metastable state to the crystalline state. The earliest work concerned with explosive crystallization found in the literature is from the year $\mathbf{1 8 5 5}$, reporting on experiments with antimony (Gore, 1855).

The process is typically initiated by locally heating the material and is then sustained by the liberation of the latent heat of fusion during the amorphous-crystalline transition, leading to a crystallization front propagating through the material. The reaction can sometimes occur in quite a dramatic fashion, exhibiting visible flashes, audible noises or surface cracking (Clevenger et al., 1990; Floro, 1986; Gore, 1855; Ma et al., 1990).

Explosive crystallization is typically examined when occuring in a thin film which is mounted on some substrate, with the crystallization front propagating in a direction parallel to the film surface. Furthermore, explosive crystallization in a film can not only proceed in a direction parallel to the film surface, but also normal to it, see e.g. Polman et al. (1989, 1990); Stolk et al. (1993); Thompson et al. (1984); Vega et al. (2005). Also, explosive crystallization in films not mounted on a substrate is examined by some authors (Marine \& Marfaing, 1991; Sharma et al., 1984). The unsupported films are first deposited onto a dissolvable substrate $(\mathrm{NaCl})$, and subsequently removed from said substrate. In Marine \& Marfaing (1991), "the films were floated off and transferred onto [electron] microscope grids".

\subsection{Heat loss influence}

Heat loss into the substrate influences the process. This influence is examined e.g. in Geiler et al. (1986); Grigoropoulos et al. (2006); Heinig \& Geiler (1985, 1986); Kurtze (1986); Rogers et al. (2006); Shklovskij \& Ostroushko (1996). The heat loss can 
even lead to the explosive crystallization process dying out Heinig \& Geiler, 1986; Provatas et al., 1996).

As a consequence of the heat loss influence, the occurence of explosive crystallization depends on the thickness of the crystallizing film, among other parameters. The concept of a minimum necessary layer thickness appears in Coffin \& Johnston (1934); Gilmer \& Leamey (1980); Johnson et al. (2008); Kurtze et al. (1984); Pumir \& Barelko (2001); Sharma et al. (1984); Shklovskij \& Kuz'menko (1989); Takamori et al. (1972). An interesting way of experimentally determining this critical thickness by using a layer of varying thickness is demonstrated in Koba \& Wickersham (1982, 1983).

\subsection{Materials}

Explosive crystallization occurs in a wide variety of materials. There is literature available on explosive crystallization in antimony (Bostanjoglo \& Schlotzhauer, 1981; Coffin, 1935a b; Coffin \& Hubley, 1950; Coffin \& Johnston, 1934; Gore, 1855; Götzberger, 1955; Kurtze et al., 1984, Shklovskij \& Kuz'menko, 1989), bismuth (Kuz'menko et al., 1991; Shklovskij \& Kuz'menko, 1989), germanium (Grigoropoulos et al., 2006; Ohdaira \& Matsumura, 2012; Rogers et al., 2006; Sharma et al., 1984; Vega et al., 2005) and silicon (Andrä et al., 1982; Endo et al., 2010; Geiler et al., 1986; Geiler \& Heinig, 1985; Götz, 1986; Knapp \& Picraux, 1981; Kuo, 2009; Ohdaira et al., 2009, 2011, 2010a,b; Polman et al., 1989, 1990; Smith et al., 2005, Spinella et al., 1998), among others.

Explosive crystallization can also occur in multi-layers made up of different materials undergoing chemical reactions (Clevenger et al., 1990; Floro, 1986; Ma et al., 1990) and in alloys (Okuda et al., 2003; Shklovskij \& Kuz'menko, 1989).

\subsection{Applications}

Areas where explosive crystallization of thin films finds applications are, for example, data storage (Okuda et al., 2003, Wickersham, 1983) or solar cell fabrication (Andrä et al., 1998; Endo et al., 2010, Ohdaira et al., 2009, 2011; Ohdaira \& Matsumura, 2012; Ohdaira et al., 2010a.b). Furthermore, advanced crystallization techniques are an area of interest for improved fabrication of thin-film displays Voutsas, 2003). 


\subsection{Motivation of present work}

The present work is concerned with the theoretical description of explosive crystallization in thin films mounted on a substrate. Specifically, the influence of heat loss into the substrate on the explosive crystallization process is examined in detail. Often, inclusion of heat loss is realized semi-empirically using an apparent heat transfer coefficient (Geiler et al., 1986, Grigoropoulos et al., 2006; Heinig \& Geiler, 1985, 1986; Kurtze, 1986; Shklovskij \& Ostroushko, 1996). In contrast, the present work derives the equations governing heat conduction into the substrate from first principles, avoiding the use of an empirical heat transfer coefficient.

This thesis builds upon, and extends, previous work on explosive crystallization in polymers done by Schneider and co-workers (Berger, 1988; Köppl, 1990; Schneider et al., 1992, 1988). Some preliminary results have already been presented previously (Buchner \& Schneider, 2010a b c c). 


\section{Problem formulation}

The process of explosive crystallization of a thin film mounted on a substrate is modeled as a crystallization zone that moves into an initially amorphous layer. Figure 2.1 shows a sketch of the problem.

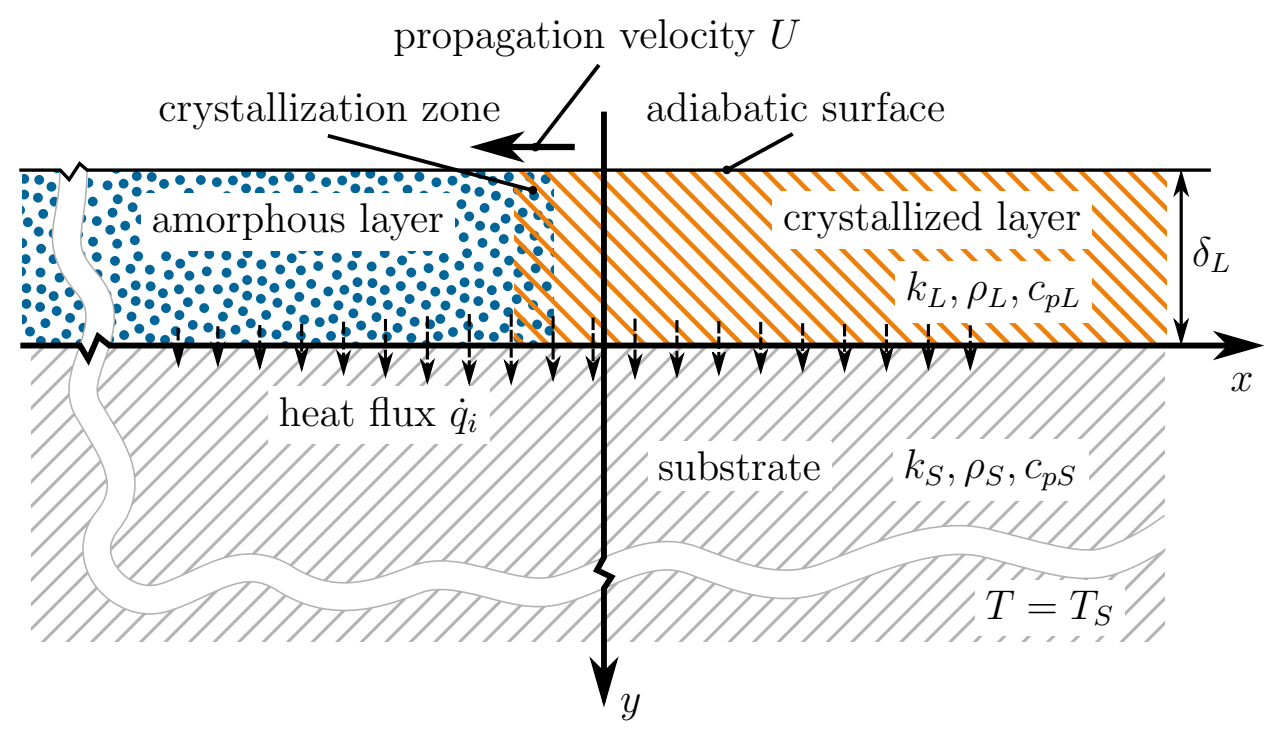

Figure 2.1.: Sketch of the problem.

It is assumed that the process is two-dimensional, i.e. depending only on the cartesian space coordinates $x$ and $y$, and on the time $t$. This is a reasonable assumption for explosive crystallization initiated by line-shaped ignition sources, which yields a linear crystallization zone, see e.g. Ohdaira et al. (2009); Rogers et al. (2006). Radial explosive crystallization, where the ignition source is a point on the film, is also reported in the literature, e.g. by Geiler et al. (1986); Takamori et al. (1972), but not considered here. Additionally, explosive crystallization can be initiated by irradiation of the whole layer with a flash lamp, cf. the articles by Ohdaira et al. The explosive crystallization process is then either of the radial type, if it is first triggered at local 
imperfections (Ohdaira \& Matsumura, 2012), or of the linear type, if it is triggered at the edge of the irradiated wafer (Ohdaira et al., 2011).

A thin layer of initially amorphous material, thickness $\delta_{L}$, is mounted on a heat conducting substrate, which is much thicker in the $y$-direction than the layer itself. Thus, the substrate may be considered a semi-infinite body. As $y \rightarrow \infty$, the temperature of the substrate tends to the initial temperature of the whole domain, $T_{S}$.

The amorphous material is transformed into crystallized material in a crystallization zone travelling with the velocity $U$. In this crystallization zone most of the crystallization takes place. Behind the crystallization zone the volume fraction of the crystalline phase, $V$, asymptotically approaches a maximum value, $V_{\infty}$, which is not necessarily 1. Polymers, in particular, are known for their inability to crystallize totally van Krevelen, 1990, p. 585). Therefore, due to a suggestion by Mandelkern (1964), the degree of crystallization $\xi$ is defined as

$$
\xi=\frac{V}{V_{\infty}}
$$

so that $\xi$ may vary from 0 very far in front of the wave to 1 very far behind the wave. $\xi=1$ means that the crystallization process has been fully completed, even if $V_{\infty}<1$.

In the course of crystallization latent heat is released, heating up the layer. Due to heat conduction, a heat flux $\dot{q}_{i}$ from the layer into the substrate occurs. Very far from the crystallization zone thermal equilibrium between the layer and the substrate is established, i.e. the temperature of the layer approaches $T_{S}$ as $x \rightarrow \pm \infty$.

The fully developed self-sustaining crystallization process shall be described as a propagating wave of invariant shape (Kluwick, 2008; Schneider, 1978) in terms of both absolute temperature, $T$, and degree of crystallization, $\xi$. This enables treating the explosive crystallization process as a steady state problem in a moving coordinate system. The coordinate system is chosen such that the wave propagates in negative $x$-direction with constant velocity $U$. 


\section{Basic equations}

\subsection{Energy equation}

Provided the crystallizing layer is sufficiently thin, the energy balance for the layer reduces to the equation

$$
\rho_{L} c_{p L} \frac{\partial T}{\partial t}=k_{L} \frac{\partial^{2} T}{\partial x^{2}}+\rho_{L} l V_{\infty} \frac{\partial \xi}{\partial t}-\frac{\dot{q}_{i}}{\delta_{L}},
$$

that is the equation of one-dimensional heat diffusion (Holman, 2010) with a source term due to the local liberation of the specific latent heat, $l$, and a heat loss term due to the interface heat flux $\dot{q}_{i}$. Convective heat losses at the free surface of the layer are neglected. Radiative heat losses are neglected, as calculations for typical experiments, e.g. for explosive crystallization of germanium, show this assumption to be justified (Sharma et al., 1984).

$k_{L}, \rho_{L}$ and $c_{p L}$ are the thermal conductivity, mass density and isobaric specific heat capacity of the layer, respectively. The same quantities are defined for the substrate, using the subscript $S$ instead of $L$. These quantities are assumed to be constant, i.e. independent of temperature and degree of crystallization.

The source term is proportional to the crystallization rate $\frac{\partial \xi}{\partial t}$, which is to be determined from a set of rate equations that follows from the kinetics of crystallization, cf. section 3.2 below.

The one-dimensional approximation in (3.1) is justified if the heat flux through the layer-substrate interface $\dot{q}_{i}$ is much smaller than the heat flux along the layer. This condition is fulfilled if (Buchner \& Schneider, 2010b)

$$
\frac{\rho_{S} c_{p S}}{\rho_{L} c_{p L}} \sqrt{\frac{\alpha_{S}}{\alpha_{L}}} \ll 1
$$


where $\alpha_{S}$ and $\alpha_{L}$ are the thermal diffusivities of the substrate and the layer, respectively. If $\rho_{S} c_{p S} / \rho_{L} c_{p L}$ is of the order of 1 , as is valid for many material combinations, this simplifies to

$$
\sqrt{\frac{\alpha_{S}}{\alpha_{L}}} \ll 1
$$

As will become clear below (see section 11.1), most of the crystallization typically happens in a small zone. In this zone, the one-dimensional approximation is not satisfied well. However, the heat flux into the substrate in this small region is small compared to the overall heat flux, so this influence can be neglected.

As far as the energy balance of the substrate is concerned, the equation to be solved in the substrate is the classical heat diffusion equation for two-dimensional unsteady heat conduction,

$$
\frac{\partial T}{\partial t}=\alpha_{S}\left(\frac{\partial^{2} T}{\partial x^{2}}+\frac{\partial^{2} T}{\partial y^{2}}\right)
$$

subject to the heat flux $\dot{q}_{i}$ at the interface as a boundary condition, cf. chapter 5 .

\subsection{Rate equations}

The main result of the crystallization theory developed by Kolmogorov (1937) and, independently, by Avrami $1939,1940,1941)$ is a multiple-integral representation of the degree of crystallization for given temperature. If the temperature is not known a priori, the kinetic equations are coupled with the energy equations, leading to a system of equations that consists of (partial) differential equations and a multipleintegral equation. This makes the problem rather intractable. However, it has been shown in Schneider et al. (1988) that the multiple-integral equation can be transformed into a system of 2, 3 or 4 (depending on the dimension of crystal growth) first-order differential equations that are of the type of chemical rate equations. This renders problems of non-isothermal crystallization, like the present one, accessible to investigation and, furthermore, provides a basis for important generalizations. Numerous applications have been found for these rate equations, see e.g. Burger et al. (2002, 2004); De Santis et al. (2005); Eder (1997); Eder \& Janeschitz-Kriegl (1997); Hütter (2001, 2004); Janeschitz-Kriegl (2010); Martins \& Cruz Pinto (2000, 2002); Waheed \& Rutledge (2005); Zuidema et al. (2001). 


\subsubsection{Rate equations for heterogeneous crystallization}

Using Avrami's model of impingement, i.e. the collision of crystals as they grow, the following system of $(m+1)$ first order differential equations for the crystallization quantity $\varphi_{0}$ is obtained (Schneider et al., 1988):

$$
\begin{aligned}
\frac{\partial \varphi_{i}}{\partial t} & =\frac{G_{C}}{L_{C}} \varphi_{i+1} \quad(i=0 \ldots m-1) \\
\frac{\partial \varphi_{m}}{\partial t} & =n_{C}\left(1-\varphi_{m}\right) .
\end{aligned}
$$

Here, $\varphi_{0}$ is defined as

$$
\varphi_{0}=-\ln (1-\xi)
$$

and $m$ is the dimensionality of crystal growth, with $m=1,2,3$ for one-, two- and three-dimensional growth, respectively. $\varphi_{1}, \varphi_{2}$ and $\varphi_{3}$ are auxiliary quantities that can be physically interpreted as being related to the total surface area, sum of the radii and numbers, respectively, of spherulites if they would grow without impingement (Eder \& Janeschitz-Kriegl, 1997). The subscript $C$ denotes the dimensional forms of crystallization-related quantities. $n_{C}$ is the probability of formation of growth nuclei per crystal nucleus and per unit time (henceforth simply called the "activation probability"). $G_{C}$ is the crystal growth velocity, while $L_{C}$ is the average distance between neighbouring crystal nuclei (Schneider et al., 1988):

$$
L_{C}=\left(\frac{V_{\infty}}{m ! \sigma \bar{N}}\right)^{1 / m}
$$

$\sigma$ is a crystal growth shape coefficient, with $\sigma=1,2 \pi, 4 \pi / 3$ for rod-like, cylindrical and spherical grains, respectively. $\bar{N}$ is the initial number of nuclei per unit volume.

Initial conditions at $t=0$ are

$$
\varphi_{i}(0)=0 \quad(i=0 \ldots m)
$$

\subsubsection{Simplification to one rate equation}

As detailed in Schneider et al. (1988), the system of rate equations reduces to a single equation for certain limiting cases. Of particular interest is the limiting case that is obtained if the activation of nuclei takes place at a much faster rate than their growth 
(i.e. $\left.n_{C} L_{C} / G_{C} \rightarrow \infty\right)$. For isothermal conditions, the system of rate equations (3.5) then simplifies to

$$
\varphi_{0}=\frac{1}{m !}\left(\frac{G_{C}}{L_{C}}\right)^{m} t^{m}
$$

Taking (3.6) into account, this is a form of the famous Avrami equation Avrami, 1939, 1940, 1941). Under non-isothermal conditions, the limiting case yields (Schneider et al., 1988)

$$
\frac{\partial \Phi}{\partial t}=\frac{G_{C}}{L_{C}}
$$

where

$$
\Phi=\left[m ! \varphi_{0}\right]^{1 / m}=[-m ! \ln (1-\xi)]^{1 / m}
$$

which in case of three-dimensional growth becomes

$$
\Phi=[-6 \ln (1-\xi)]^{1 / 3}
$$

\subsubsection{Rate equations for homogeneous crystallization}

In contrast to heterogenous crystallization, where pre-existing crystal nuclei are activated and begin to grow, there are no nuclei at the beginning of homogeneous crystallization. Through random fluctuations, nuclei are spontaneously formed in the amorphous material with a certain nucleation rate $I_{C}$ (see section 3.4), and immediately begin to grow. This process is also sometimes called "random nucleation and growth" (Olson \& Roth, 1988).

Keeping this difference in mind, the derivation of the rate equations is analogous to the procedure for heterogenous crystallization. The key points are reproduced here, but a more detailed derivation can be found in appendix A.1.

The symbols $\varphi$ and $\phi$ are used to distinguish between heterogeneous and homogeneous crystallization, respectively. Using the crystallization variable defined in (3.6) in an equivalent form

$$
\phi_{C, 0}=\varphi_{0}=-\ln (1-\xi)
$$


one arrives at the system of equations

$$
\begin{aligned}
\frac{\partial \phi_{C, i}}{\partial t} & =G_{C} \phi_{C, i+1} \quad(i=0 \ldots m-1) \\
\frac{\partial \phi_{C, m}}{\partial t} & =\frac{m ! \sigma}{V_{\infty}} I_{C} .
\end{aligned}
$$

Here, there is no characteristic length scale like $L_{C}$ in case of heterogeneous crystallization. Consequently, the quantities $\phi_{C, i}(i=1 \ldots m)$ now have the dimension length $^{-\mathrm{i}}$.

The initial conditions are identical to the initial conditions for heterogeneous crystallisation, i.e.

$$
\phi_{C, i}(0)=0 \quad(i=0 \ldots m) .
$$

\subsection{Crystal growth velocity}

The crystal growth velocity $G_{C}$ is the direction-averaged linear growth velocity of crystals. A variety of expressions for the temperature dependence of $G_{C}$ may be found in the literature. A relationship that is often used, in particular for polymers, cf. Janeschitz-Kriegl (2010), was derived by Hoffman (1964) on the basis of earlier work by Williams et al. (1955). It is consistent with thermodynamics for all temperatures $T$, and will be adapted for other materials in what follows. The relationship reads:

$$
G_{C}(T)= \begin{cases}G_{0} \exp \left[-\frac{E_{1}}{k_{B}\left(T-T_{g}\right)}\right] \exp \left[-\frac{E_{2} T_{m}}{k_{B} T\left(T_{m}-T\right)}\right] & \text { for } T_{g}<T<T_{m} \\ 0 & \text { for } T \leq T_{g} ; T \geq T_{m}\end{cases}
$$

where $G_{0}$ is a characteristic value of the crystal growth velocity, $E_{1}$ and $E_{2}$ are kinetic constants that characterize activation energies, and $k_{B}=8.617332 \times 10^{-5} \mathrm{eV} / \mathrm{K}$ is the Boltzmann constant. $T_{g}$ and $T_{m}$ denote the glass transition temperature and the melting temperature of the material, respectively. $G_{C}$ vanishes exponentially as $T \rightarrow T_{g}$ and $T \rightarrow T_{m}$, respectively. In accordance with common practice, the growth velocity is taken to be zero above the melting temperature as well as below the glass transition temperature. 
Another formulation for $G_{C}$ is based on using an Arrhenius dependency of the form

$$
f(T)=f_{0} \exp \left[-\frac{E}{k_{B} T}\right]
$$

in the first exponential term in (3.16), which is dominant at low temperatures. In this case, $G_{C}$ is defined as

$$
G_{C}(T)= \begin{cases}G_{0} \exp \left[-\frac{E_{G}}{k_{B} T}\right] \exp \left[-\frac{E_{G m} T_{m}}{k_{B} T\left(T_{m}-T\right)}\right] & \text { for } T_{g}<T<T_{m} \\ 0 & \text { for } T \leq T_{g} ; T \geq T_{m}\end{cases}
$$

where $E_{G}$ and $E_{G m}$ are activation energies. Note that with an Arrhenius dependency, $G_{C} \neq 0$ at the glass transition temperature. In fact, the glass transition temperature does not enter the equation at all. To be able to treat the problem as a propagating wave of invariant shape, it is necessary that $G_{C} \equiv 0$ below a temperature slightly above $T_{S}$. For convenience, this temperature is also designated as $T_{g}$. Additionally, $G_{C} \equiv 0$ for a temperature above $T_{m}$, as in 3.16 .

Often, only values for $E$ and $f_{0}$ from (3.17) can be found in the literature, because a pure Arrhenius dependency is assumed for the crystal growth velocity (Donovan et al., 1983; Farjas \& Roura, 2006; Götzberger, 1955; Heinig \& Geiler, 1985; Johnson et al., 2008; Knapp \& Picraux, 1981; Olson \& Roth, 1988; Shklovskij \& Kuz'menko, 1989; Sontheimer et al., 2009). This is often used because the higher temperature regime, where deviations from this dependency become apparent, is either difficult to measure or not even accessible to experimental measurements, see e.g. Marine \& Marfaing (1991).

When using (3.18), the relations

$$
\begin{gathered}
E=E_{G}+E_{G m}, \\
f_{0}=G_{0} \exp \left[-\frac{E_{G m}}{k_{B} T_{m}}\right],
\end{gathered}
$$

can be used to obtain values for $G_{0}$ and $E_{G}$ from a given or assumed $E_{G m}$ and experimentally available $E$ and $f_{0}$, such that the crystal growth velocity values at low temperatures remain essentially unchanged compared to 3.17).

Using (3.18), $G_{C}(T)$ has a maximum value at a certain temperature $T^{*}$, i.e.

$$
G_{C}\left(T^{*}\right)=\max \left[G_{C}(T)\right]
$$


The relative sizes of $E_{G}$ and $E_{G m}$ decide the location of $T^{*}$ and the temperature where a deviation from the pure Arrhenius behaviour becomes noticeable. Therefore, with no other data available, the temperature range where measurements of $G_{C}$ are available places an upper limit on $E_{G m}$.

\subsection{Crystal nucleation rate}

Based on the classical Becker-Volmert nucleation theory, it can be shown (Köster \& Herold, 1981) that for large undercooling, i.e. far away from the melting temperature, the expression for the homogeneous crystal nucleation rate is dominated by an Arrhenius-type dependency of the form (3.17).

Taking the full temperature domain into account, this expression is assumed to be equivalent to 3.18 :

$$
I_{C}(T)= \begin{cases}I_{0} \exp \left[-\frac{E_{I}}{k_{B} T}\right] \exp \left[-\frac{E_{I m} T_{m}}{k_{B} T\left(T_{m}-T\right)}\right] & \text { for } T_{g}<T<T_{m} \\ 0 & \text { for } T \leq T_{g} ; T \geq T_{m}\end{cases}
$$

where $E_{I}$ and $E_{I m}$ are activation energies for nucleation. Furthermore, it can be safely assumed that no nucleation takes place below the glass transition temperature $T_{g}$ (Köster \& Herold, 1981).

Just as is the case for the crystal growth velocity, sometimes only a pure Arrheniusstyle dependency like (3.17) is considered in the literature. This is especially true for experimental papers, because the higher temperature regime, where deviations from (3.17) become apparent, is not accessible to experimental measurements (Farjas \& Roura, 2006; Olson \& Roth, 1988; Sontheimer et al., 2009). Sometimes, though, authors try to reconcile both relations, using (3.17) with experimental data, but also considering bell-shaped curves (Marine \& Marfaing, 1991).

Analogously to the crystal growth velocity, values obtained using a pure Arrheniusstyle dependency can be converted for use in (3.21), while maintaining the lowtemperature behaviour. This can be achieved by using (3.19) and replacing the crystal growth velocity quantities with their equivalent counterparts for the crystal nucleation rate. 


\subsection{Crystallization time}

Sometimes, the parameters relevant for determining the crystal nucleation rate are not directly available for measurement in an experiment. In this case, researchers often measure the crystallization time, which is more accessible, e.g. by using calorimetry (e.g. Martins \& Cruz Pinto, 2002), electrical resistivity (e.g. Shklovskij \& Kuz'menko, 1989), or optical measurements (Olson \& Roth, 1988). The nucleation and the growth components can be separated through use of kinetic theory as follows (adapted from Olson \& Roth, 1988):

Assuming three-dimensional growth and isothermal conditions, i.e. constant crystal nucleation rate and constant growth velocity of crystallites, the degree of crystallization follows the famous Avrami equation (Avrami, 1939, 1940, 1941)

$$
\xi(t)=1-\exp \left[-(t / \tau)^{4}\right]
$$

The crystallization time $\tau$ is the time required to reach a degree of crystallization of $1-1$ e. It is often assumed to vary with temperature according to the relation

$$
\tau(T)=\tau_{0} \exp \left[\frac{E_{\tau}}{k_{B} T}\right]
$$

This assumption is confirmed by measurements over a wide range of experimentally accessible temperatures. For purely isotropic, three-dimensional growth, the relation

$$
\tau \propto\left(I_{C} G_{C}^{3}\right)^{-1 / 4}
$$

is given by (Olson \& Roth, 1988). Experiments show that it can be assumed that crystal growth remains largely isotropic even if the crystallites grow big enough to touch both film surfaces (Olson \& Roth, 1988).

If A.7) is used with the above assumptions, one arrives at a more precise version of (3.24), in accordance with the Avrami equation (Janeschitz-Kriegl, 2010):

$$
\tau=\left(\frac{\sigma}{4 V_{\infty}} I_{C} G_{C}^{3}\right)^{-1 / 4}
$$

Inserting Arrhenius dependencies, i.e. according to (3.17), for $I_{C}$ and $G_{C}$ in (3.25), the relationship

$$
4 E_{\tau}=E_{I}+3 E_{G}
$$


between the activation energies of crystallisation time, crystal nucleation, and crystal growth is obtained. This demonstrates that, using (3.25) and (3.26), the crystal nucleation rate $I_{C}$ can be fully characterised even if only the crystal growth velocity $G_{C}$ and crystallization time $\tau$ are known. Fortunately, enough nucleation data were found for a comparison to experiments, see chapter 11, so using the presented approach was not necessary. However, this technique might be useful to other researchers, and so it is included here. 


\section{Non-dimensional formulation}

\subsection{Non-dimensional variables}

To arrive at a proper non-dimensional formulation of the problem, first some characteristic quantities have to be identified (Buchner \& Schneider, 2010a b):

The total end temperature $T_{t o t}$ is the temperature that would be attained by releasing all the latent heat of crystallization of the amorphous material without considering a possible melting transition at $T_{m}$ :

$$
T_{t o t}=T_{S}+\frac{V_{\infty} l}{c_{p L}}
$$

$T_{\text {tot }}$ is not necessarily identical to $T_{a d}$, the final temperature in the adiabatic case, which is constrained by encountering the melting transition at $T_{m}$, and defined as

$$
T_{a d}=\min \left[T_{t o t}, T_{m}\right]
$$

Quantities concerning an adiabatic process are denoted by the subscript ad.

In view of the physical meaning of $T_{\text {tot }}$, it makes sense to introduce the nondimensional temperature difference as

$$
\Theta=\frac{T-T_{S}}{T_{t o t}-T_{S}}
$$

so that $0 \leq \Theta \leq 1$. Consequently, the non-dimensional glass transition and melting temperatures, respectively, become

$$
\Theta_{g}=\frac{T_{g}-T_{S}}{T_{t o t}-T_{S}} ; \quad \Theta_{m}=\frac{T_{m}-T_{S}}{T_{t o t}-T_{S}}
$$


Using the wave propagation speed $U$, which is to be determined as part of the solution, as a characteristic velocity, the reference length for heat conduction and the reference heat flux, respectively, are defined as

$$
\begin{aligned}
& L_{r e f}=\frac{k_{L}}{U \rho_{L} c_{p L}}, \\
& \dot{q}_{r e f}=U \rho_{L} V_{\infty} l \frac{e_{S}}{e_{L}} .
\end{aligned}
$$

The abbreviation $e$ is defined as

$$
e=\sqrt{k \rho c_{P}}
$$

and is important e.g. when describing the thermal contact of semi-infinite bodies.

A reference time scale for the kinetics of the explosive crystallization process, $t_{C, \text { ref }}$ is also needed. For isothermal crystallization, a characteristic time scale can be defined as

$$
\begin{aligned}
& t_{C}(T)=\frac{L_{C}}{G_{C}(T)}, \text { for heterogeneous crystallization with immediate activation; } \\
& t_{C}(T)=\left[G_{C}(T)^{3} I_{C}(T)\right]^{-1 / 4}, \text { for homogeneous crystallization. }
\end{aligned}
$$

Due to the different formulations of the rate equations, the definition of this time scale is different for heterogeneous and homogeneous crystallization 11 For homogeneous crystallization, $t_{C}(T)$ is, except for a multiplicative factor, identical to $\tau$, the time of crystallization in the Avrami equation (3.25).

For the explosive (and non-isothermal) crystallization process, the physically most meaningful (i.e. dominant) reference time scale is the time scale of the fastest crystallization occuring in the whole domain. Thus, the appropriate reference temperature $T_{\text {ref }}$ is defined as the temperature where $t_{C}(T)$ is minimal:

$$
\begin{aligned}
t_{C}\left(T_{r e f}\right) & =\min \left(t_{C}(T)\right), \text { with } T_{S} \leq T \leq T_{a d} \\
\Theta_{r e f} & =\frac{T_{r e f}-T_{S}}{T_{t o t}-T_{S}}
\end{aligned}
$$

\footnotetext{
${ }^{1}$ This is to avoid a dual definition of $H, \lambda$ for heterogeneous and homogeneous crystallization, respectively, see 4.27 and 4.28.
} 
In case of a pure Arrhenius dependency, cf. (3.17), or for subcritical waves (see section 4.2), $T_{r e f}=T_{a d}$, and the distinction between the two temperatures seems overly complicated. In case of supercritical waves, $T_{\text {ref }}$ and $T_{a d}$ differ, and using $T_{r e f}=T_{a d}$ would become problematic as it is possible that $t_{C}\left(T_{a d}\right) \rightarrow \infty$ as $T_{a d} \rightarrow T_{m}$. Thus, it is preferable to use the slightly more complicated formulation 4.9 .

Now that $T_{r e f}$ is properly defined, a definition of $t_{C, r e f}$ is straightforward:

$$
t_{C, r e f}=t_{C}\left(T_{r e f}\right)
$$

The reference quantities for the crystal growth velocity, activation probability and crystal nucleation rate, respectively, are defined as:

$$
\begin{aligned}
G_{C, \text { ref }} & =G_{C}\left(T_{r e f}\right), \\
n_{C, \text { ref }} & =n_{C}\left(T_{r e f}\right), \\
I_{C, \text { ref }} & =I_{C}\left(T_{r e f}\right) .
\end{aligned}
$$

A non-dimensional interface heat flux, crystal growth velocity, activation probability and crystal nucleation rate, respectively, are then defined as

$$
\begin{aligned}
\dot{q} & =\frac{\dot{q}_{i}}{\dot{q}_{r e f}}, \\
G & =\frac{G_{C}}{G_{C, r e f}}, \\
n & =\frac{n_{C}}{n_{C, r e f}}, \\
I & =\frac{I_{C}}{I_{C, r e f}} .
\end{aligned}
$$

Finally, since the aim of the analysis is to find solutions describing propagating waves of invariant shape, the non-dimensional wave coordinate $\eta$ is introduced, which describes a space coordinate in a reference frame moving with velocity $U$ :

$$
\eta=\frac{x+U t}{L_{r e f}}
$$




\subsection{Classification of crystallization waves}

The existence of a maximum in the crystal growth velocity has important consequences for the structure of the crystallization wave. For the adiabatic case it has been shown previously (Köppl, 1990; Schneider, 1996) that the structure of the crystallization wave depends on whether $T_{a d}<T^{*}$ ("subcritical wave"), $T_{a d} \approx T^{*}$ ("critical wave") or $T_{a d}>T^{*}$ ("supercritical wave"), where $T_{a d}$ is the temperature attained behind the wave in an adiabatic process, as defined in (4.2).

When using more than one rate equation, this classification is not entirely sufficient. Instead of the temperature of the maximum crystal growth velocity $T^{*}$, it is more appropriate to use the temperature of a minimum characteristic time of crystallization, $T_{r e f}$, as defined in 4.9 . This is because the crystal growth velocity is not the only kinetic quantity responsible for crystallization any more, which is in contrast to the assumption of instant activation of nuclei in Köppl (1990); Schneider (1996).

In addition to $T_{a d}$, another quantity important for the classification of crystallization waves is $T_{t o t} \geq T_{a d}$, the temperature attained by releasing all the latent heat of crystallization of the amorphous material, as defined in 4.1). In case of heat losses due to conduction of heat into the substrate, $T_{\text {tot }}$ may be above $T_{m}$, but the temperature in the non-adiabatic wave remains below $T_{m}$ and approaches $T_{S}$ far behind the wave.

Similarly, a wave could be classified as supercritical (i.e. $T_{a d}>T_{r e f}$ ), but due to heat losses the temperature in the wave never reaches $T_{a d}$ and remains effectively subcritical, cf. section 11.3 .3 .

\subsection{Non-dimensional formulation of the crystal growth velocity}

In non-dimensional form, (3.16) becomes (Buchner \& Schneider, 2010b)

$$
G(\Theta)=\left\{\begin{array}{cc}
\exp \left\{-\frac{C_{1}\left(\Theta_{r e f}-\Theta\right)}{\left(\Theta_{r e f}-\Theta_{g}\right)\left(\Theta-\Theta_{g}\right)}\right\} \exp \left\{C_{2}\left(C_{3}+\Theta_{m}\right)\right. & \text { for } \Theta_{g}<\Theta<\Theta_{m}, \\
\left.\cdot\left[\frac{1}{\Theta_{m}-\Theta_{r e f}}-\frac{C_{4}}{\left(C_{3}+\Theta\right)\left(\Theta_{m}-\Theta\right)}\right]\right\} & \text { for } \Theta \leq \Theta_{g} ; \Theta \geq \Theta_{m},
\end{array}\right.
$$


with

$$
\begin{aligned}
& C_{1}=\frac{c_{p L} E_{1}}{k_{B} V_{\infty} l}, \quad C_{2}=\frac{E_{2}}{k_{B} T_{r e f}}, \\
& C_{3}=\frac{c_{p L} T_{S}}{V_{\infty} l} \quad C_{4}=\frac{c_{p L} T_{r e f}}{V_{\infty} l} .
\end{aligned}
$$

In the case of low-temperature Arrhenius behaviour, cf. (3.18), the non-dimensional formulation can be derived from 4.20 and 4.21 by replacing $\Theta_{g}$ with $-C_{3}, E_{1}$ with $E_{G}$, and $E_{2}$ with $E_{G m}$ :

$$
G(\Theta)=\left\{\begin{array}{cc}
\exp \left\{-\frac{C_{1, G}\left(\Theta_{r e f}-\Theta\right)}{\left(\Theta_{r e f}+C_{3}\right)\left(\Theta+C_{3}\right)}\right\} \exp \left\{C_{2, G}\left(C_{3}+\Theta_{m}\right)\right. \\
\left.\cdot\left[\frac{1}{\Theta_{m}-\Theta_{r e f}}-\frac{C_{4}}{\left(C_{3}+\Theta\right)\left(\Theta_{m}-\Theta\right)}\right]\right\} & \text { for } \Theta_{g}<\Theta<\Theta_{m}, \\
0 & \text { for } \Theta \leq \Theta_{g} ; \Theta \geq \Theta_{m},
\end{array}\right.
$$

where $C_{1}$ and $C_{2}$ have been replaced by

$$
C_{1, G}=\frac{c_{p L} E_{G}}{k_{B} V_{\infty} l}, \quad C_{2, G}=\frac{E_{G m}}{k_{B} T_{r e f}}
$$

The functions $G(\Theta)$ according to 4.20 and 4.22 , respectively, are shown in fig. 4.1 , for parameter values as in Buchner \& Schneider (2010b). The value for $C_{1, G}$ in 4.22 has been adjusted to match the peak location and high-temperature behaviour of both curves.

\subsection{Non-dimensional formulation of the crystal nucleation rate}

The non-dimensional formulation of the crystal nucleation rate (3.21) resembles 4.22 and 4.23):

$I(\Theta)=\left\{\begin{array}{cc}\exp \left\{-\frac{C_{1, I}\left(\Theta_{r e f}-\Theta\right)}{\left(\Theta_{r e f}+C_{3}\right)\left(\Theta+C_{3}\right)}\right\} \exp \left\{C_{2, I}\left(C_{3}+\Theta_{m}\right)\right. & \\ \left.\cdot\left[\frac{1}{\Theta_{m}-\Theta_{r e f}}-\frac{C_{4}}{\left(C_{3}+\Theta\right)\left(\Theta_{m}-\Theta\right)}\right]\right\} & \text { for } \Theta_{g}<\Theta<\Theta_{m}, \\ 0 & \text { for } \Theta \leq \Theta_{g} ; \Theta \geq \Theta_{m},\end{array}\right.$ 

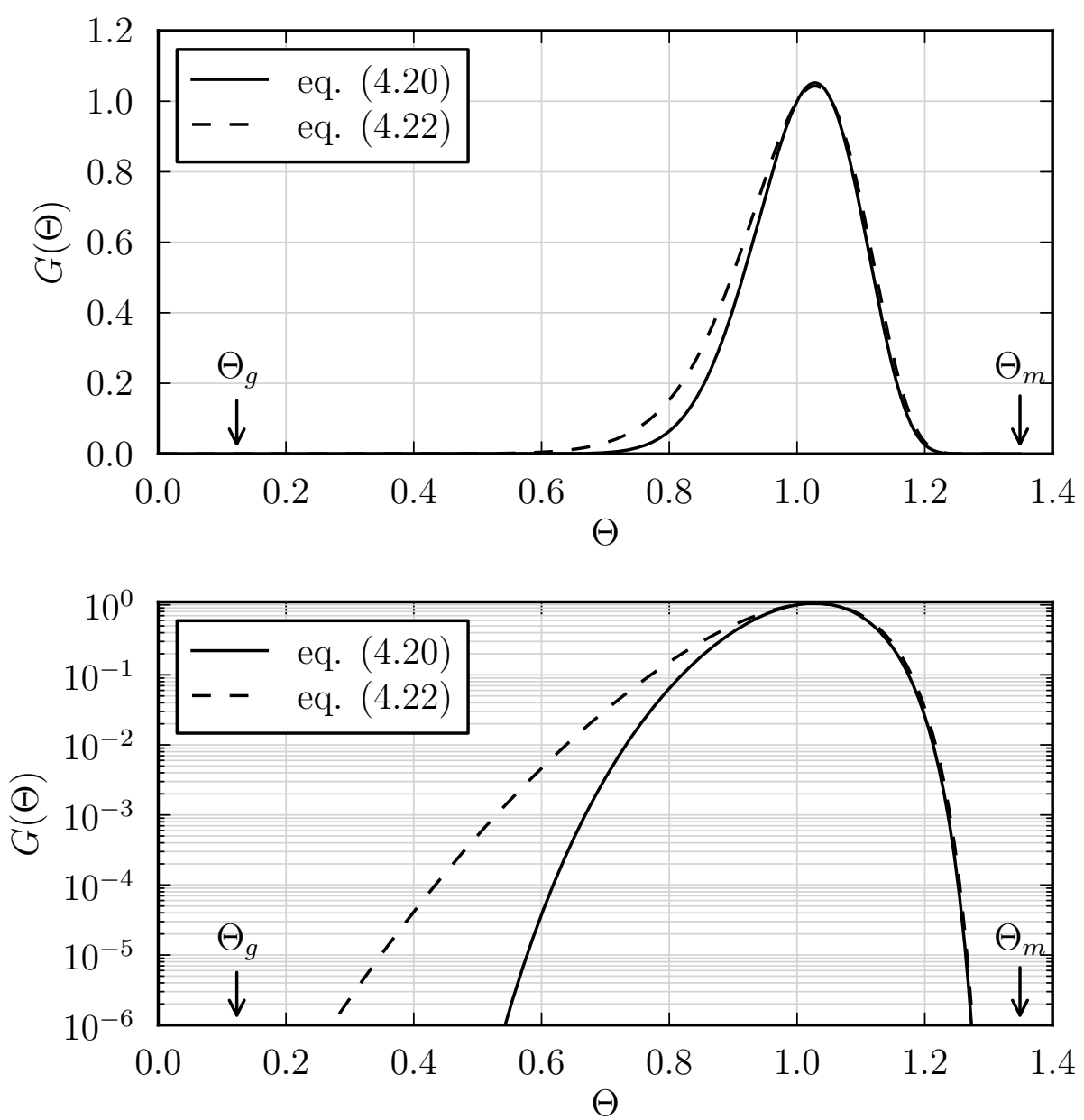

Figure 4.1.: Non-dimensional crystal growth velocity according to 4.20 and 4.22). Parameter values from Buchner \& Schneider $(2010 b): C_{1}=13.23, C_{2}=$ $0.660, C_{3}=1.564, C_{4}=2.564, \Theta_{g}=0.123, \Theta_{m}=1.349$. In 4.22, $C_{1, G}=108.75$, and $C_{2, G}=C_{2}$.

where

$$
C_{1, I}=\frac{c_{p L} E_{I}}{k_{B} V_{\infty} l}, \quad C_{2, I}=\frac{E_{I m}}{k_{B} T_{r e f}} .
$$




\subsection{Crystallization wave of invariant shape}

\subsubsection{Energy equation and non-dimensional parameters}

It is assumed that all non-dimensional dependent variables depend on $\eta$ only. Using the non-dimensional variables defined above, the energy equation (3.1) becomes, as given by Buchner \& Schneider (2010b),

$$
\frac{\mathrm{d} \Theta}{\mathrm{d} \eta}=\frac{\mathrm{d}^{2} \Theta}{\mathrm{d} \eta^{2}}+\frac{\mathrm{d} \xi}{\mathrm{d} \eta}-H \lambda \dot{q}
$$

with

$$
\begin{aligned}
H & =\frac{\rho_{S} c_{p, S}}{\rho_{L} c_{p, L}} \frac{1}{\delta_{L}} \sqrt{\alpha_{S} t_{C, r e f}} \\
\lambda & =\frac{1}{U} \sqrt{\frac{\alpha_{L}}{t_{C, r e f}}}
\end{aligned}
$$

$H$ is a non-dimensional parameter characterizing the influence of heat loss into the substrate. Its definition has been generalized from (Buchner \& Schneider, 2010b) to additionally accomodate homogeneous crystallization by using 4.11.

Note that the set of non-dimensional parameters has been defined such that the unknown propagation velocity $U$ appears only in one parameter, i.e $\lambda$, which therefore plays the role of an eigenvalue. $\lambda^{2}$ can be interpreted as a ratio of the characteristic time of heat conduction to the characteristic time of crystallization, and therefore a (first) Damköhler number (Köppl, 1990).

Furthermore, note that the definition of $t_{C, r e f}$ is different for heterogenous and homogeneous crystallization, see 4.8a) and 4.8b), respectively. 


\subsubsection{Heterogeneous crystallization}

The full set of rate equations for heterogeneous crystallization 3.5 becomes:

$$
\begin{aligned}
\frac{\mathrm{d} \xi}{\mathrm{d} \eta} & =\lambda^{2} G(\Theta) \varphi_{1}(\eta)(1-\xi) \\
\frac{\mathrm{d} \varphi_{1}}{\mathrm{~d} \eta} & =\lambda^{2} G(\Theta) \varphi_{2}(\eta) \\
\frac{\mathrm{d} \varphi_{2}}{\mathrm{~d} \eta} & =\lambda^{2} G(\Theta) \varphi_{3}(\eta) \\
\frac{\mathrm{d} \varphi_{3}}{\mathrm{~d} \eta} & =\lambda^{2} \mu n(\Theta)\left[1-\varphi_{3}(\eta)\right],
\end{aligned}
$$

where the non-dimensional parameter $\mu$ is defined as

$$
\mu=\frac{n_{C, r e f} L_{C}}{G_{C, r e f}} .
$$

\subsubsection{Single rate equation}

The single rate equation in case of immediate activation of nuclei $(3.12)$ becomes

$$
\frac{\mathrm{d} \xi}{\mathrm{d} \eta}=\lambda^{2} G(\Theta) g(\xi)
$$

with the auxiliary function $g(\xi)$ defined as

$$
g(\xi)=(9 / 2)^{1 / 3}(1-\xi)[-\ln (1-\xi)]^{2 / 3}
$$

\subsubsection{Homogeneous crystallization}

The absence of a characteristic length scale for crystallization, and the dimensionality of the $\phi_{C, i}$, complicates matters in case of homogeneous crystallization. The crystallization variables $\phi_{C, i}$ are made non-dimensional using the expression

$$
\phi_{i}=\left(\frac{G_{C, r e f}}{I_{C, r e f}}\right)^{i / 4} \phi_{C, i} \quad(i=0 \ldots m) .
$$


Consequently, (3.14) becomes

$$
\begin{aligned}
\frac{\mathrm{d} \xi}{\mathrm{d} \eta} & =\lambda^{2} G(\Theta) \phi_{1}(\eta)(1-\xi) \\
\frac{\mathrm{d} \phi_{1}}{\mathrm{~d} \eta} & =\lambda^{2} G(\Theta) \phi_{2}(\eta) \\
\frac{\mathrm{d} \phi_{2}}{\mathrm{~d} \eta} & =\lambda^{2} G(\Theta) \phi_{3}(\eta) \\
\frac{\mathrm{d} \phi_{3}}{\mathrm{~d} \eta} & =\lambda^{2} M I(\Theta)
\end{aligned}
$$

with the non-dimensional parameter $M$ defined as

$$
M=\frac{6 \sigma}{V_{\infty}}
$$

(4.34) is identical to 4.29, except for the last equation, 4.34d). Furthermore, in contrast to $\varphi_{1}-\varphi_{3}$, the values of $\phi_{1}-\phi_{3}$ do not remain within the range $[0,1]$, but reach higher values. 


\section{Treatment of substrate}

In the substrate, the heat diffusion equation for two-dimensional unsteady heat conduction (3.4), subject to the heat flux $\dot{q}_{i}$ at the interface as a boundary condition, is solved.

\subsection{Integral representation of the temperature in the substrate}

Among the particular solutions of the heat diffusion equation there is the well-known solution for a line heat source moving with constant velocity through an infinite medium, cf. Grigull \& Sandner (1984, p. 131 ff.). Here, the solution is used to describe the temperature field in the substrate in terms of a continuous distribution of moving heat sources $\dot{q}(\eta)$ at the interface. This gives (Buchner \& Schneider, 2010b) the following integral representation for the temperature in the substrate in terms of the unknown source distribution $\dot{q}(\eta)$ :

$$
\Theta(\eta, Y)=\frac{1}{\pi \sqrt{a}} \int_{-\infty}^{+\infty} \dot{q}(s) \exp \left(\frac{\eta-s}{2 a}\right) \mathrm{K}_{0}\left(\frac{1}{2 a} \sqrt{(\eta-s)^{2}+Y^{2}}\right) \mathrm{d} s
$$

where $Y=y / L_{r e f}$, while $\mathrm{K}_{0}$ denotes the modified Bessel function of the second kind (Olver et al., 2010). The dimensionless parameter $a$ is defined as the ratio of the thermal diffusivities of substrate and layer, respectively:

$$
a=\frac{\alpha_{S}}{\alpha_{L}}
$$

At the interface, i.e. at $Y=0,5.1$ reduces to

$$
\Theta(\eta)=\frac{1}{\pi \sqrt{a}} \int_{-\infty}^{+\infty} \dot{q}(s) \exp \left(\frac{\eta-s}{2 a}\right) \mathrm{K}_{0}\left(\frac{|\eta-s|}{2 a}\right) \mathrm{d} s .
$$


Assuming perfect thermal contact between substrate and layer, $\Theta(\eta)$ in $(5.3)$ must be equal to the temperature appearing in the energy equation and in the rate equation(s). For a formulation including imperfect thermal contact see section 5.3 .

Thus, the complete set of equations consists of the ordinary differential equation (4.26), the integral equation (5.3), and one or more rate equation(s), i.e. 4.29), 4.31) or 4.34, for the unknown functions $\Theta(\eta), \dot{q}(\eta), \xi(\eta)$, and $\varphi_{1}-\varphi_{3}$ or $\phi_{1}-\phi_{3}$, depending on the chosen set of rate equations.

The solution has to satisfy the following boundary conditions far ahead of, and far behind, the wave, respectively:

$$
\begin{array}{ll}
\Theta=0, \quad \xi=0 & \text { as } \eta \rightarrow-\infty, \\
\Theta=0 & \text { as } \eta \rightarrow+\infty,
\end{array}
$$

and, if required,

$$
\varphi_{1}=\varphi_{2}=\varphi_{3}=0 \text { or } \phi_{1}=\phi_{2}=\phi_{3}=0 \quad \text { as } \eta \rightarrow-\infty \text {. }
$$

Note that in the absence of a substrate, i.e. under adiabatic conditions, the temperature far behind the wave has to satisfy the condition $\Theta=1$ as $\eta \rightarrow+\infty$.

The problem formulated herewith is an eigenvalue problem, i.e. non-trivial solutions for homogeneous boundary conditions are to be found. The eigenvalue $\lambda$ determines the propagation speed of the wave, $U$, cf. (4.28). The (numerical) solution of the above system of differential equations and a coupled integral equation appears rather cumbersome. However, substantial simplifications are possible, as will be shown in the next sections.

\subsection{Expansion for weakly conducting substrates}

\subsubsection{Expansion of the integral representation for small values of a}

In many cases of practical interest, e.g. for germanium on quartz (Grigoropoulos et al. 2006 ) or for silicon on quartz (Heinig \& Geiler, 1985), the thermal diffusivity of the substrate is much smaller than the thermal diffusivity of the crystallizing layer, i.e. $a \ll 1$ according to 5.2 . 
An asymptotic expansion of (5.3) for small values of $a$ is not straightforward, however. The basic idea is to take $\dot{q}$ out of the integral of (5.3). To this end, the integral $(5.3)$ is first transformed with the substitution

$$
\varsigma=-\frac{\eta-s}{2 a}
$$

This gives

$$
\Theta(\eta)=\frac{2 \sqrt{a}}{\pi} \int_{-\infty}^{+\infty} \dot{q}(\eta+2 a \varsigma) \exp (-\varsigma) \mathrm{K}_{0}(|\varsigma|) \mathrm{d} \varsigma
$$

In view of the known asymptotic behavior of $\mathrm{K}_{0}$ (Olver et al., 2010)

$$
\mathrm{K}_{0}(\varsigma)=\sqrt{\frac{\pi}{2 \varsigma}} \exp (-\varsigma)\left(1-\frac{1}{8 \varsigma}+\ldots\right) \text { as } \varsigma \rightarrow \infty,
$$

the integral in (5.8) is then split into three parts:

$$
\begin{aligned}
\frac{\pi}{2 \sqrt{a}} \Theta(\eta) & =\int_{-\infty}^{0} \dot{q}(\eta+2 a \varsigma) \overbrace{\left[\exp (-\varsigma) \mathrm{K}_{0}(|\varsigma|)-\sqrt{\left.\frac{\pi}{2|\varsigma|}\right]}\right.}^{\mathrm{A}} \mathrm{d} \varsigma \\
& +\int_{-\infty}^{0} \dot{q}(\eta+2 a \varsigma) \sqrt{\sqrt{\frac{\pi}{2|\varsigma|}} \mathrm{d} \varsigma} \\
& +\int_{0}^{+\infty} \dot{q}(\eta+2 a \varsigma) \underbrace{\exp (-\varsigma) \mathrm{K}_{0}(|\varsigma|)}_{\mathrm{B}} \mathrm{d} \varsigma .
\end{aligned}
$$

According to $(5.9)$, the kernels $\mathrm{A}$ and $\mathrm{B}$ in the first integral and in the third integral, respectively, decay quickly as $\varsigma \rightarrow \mp \infty$. Figure 5.1 shows a plot of A and B and their asymptotic behaviour for $|\varsigma| \gg 1$.

For $\varsigma=O(1)$, the term $2 a \varsigma$ in the argument of $\dot{q}$ may be neglected because $a \ll 1$. For $|\varsigma| \gg 1$, on the other hand, the term $2 a \varsigma$ may be neglected because the quick decay of the integral kernels ensures that the resulting error stays small. As a result, $\dot{q}(\eta+2 a \varsigma)$ may be replaced by $\dot{q}(\eta)$ and, therefore, be taken out of the integrals. The remaining integrals can then be evaluated:

$$
\begin{aligned}
& \int_{-\infty}^{0}\left[\exp (-\varsigma) K_{0}(|\varsigma|)-\sqrt{\frac{\pi}{2|\varsigma|}}\right] d \varsigma=+1 ; \\
& \int_{0}^{+\infty} \exp (-\varsigma) K_{0}(|\varsigma|) d \varsigma=-1 \text {. }
\end{aligned}
$$




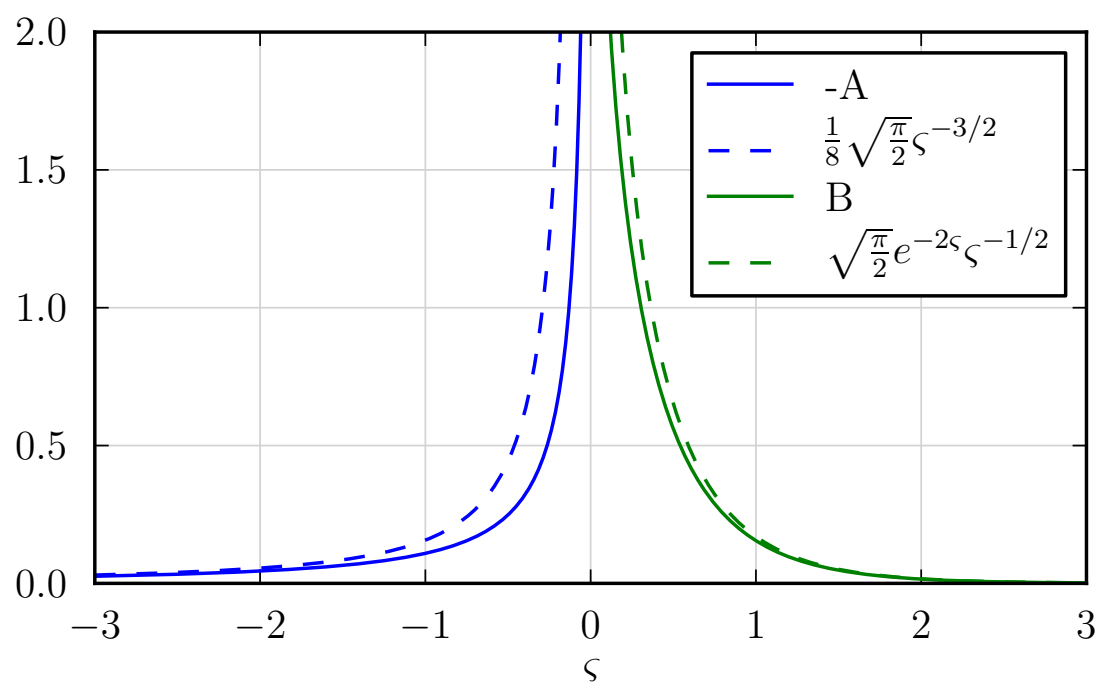

Figure 5.1.: Behaviour of integral kernels A and B.

Hence the first and the third term on the right hand side of 5.10 cancel out as $a \rightarrow 0$. The remaining integral, after another transformation according to (5.7), gives

$$
\Theta(\eta)=\frac{1}{\sqrt{\pi}} \int_{-\infty}^{\eta} \frac{\dot{q}(s)}{\sqrt{\eta-s}} \mathrm{~d} s
$$

5.13 is a coupling condition between the temperature and the heat flux at the interface.

\subsubsection{Inversion of the interface coupling condition}

(5.13) is of the form of Abel's integral equation (Polyanin \& Manzhirov, 1999). It can be inverted to give

$$
\dot{q}(\eta)=\frac{1}{\sqrt{\pi}} \frac{\mathrm{d}}{\mathrm{d} \eta} \int_{-\infty}^{\eta} \frac{\Theta(s)}{\sqrt{\eta-s}} \mathrm{~d} s
$$

Equation (5.14) shows that the local heat transfer rate depends on the temperature distribution up to infinitely far ahead of the position that is considered. This is in contrast to any formulation in terms of a local heat transfer coefficient, which is often found in the literature, e.g. in Geiler et al. (1986); Grigoropoulos et al. (2006); Heinig \& Geiler (1985, 1986); Kurtze (1986); Shklovskij \& Ostroushko (1996). It 
is also at odds with a more general polynomial relationship between heat loss and temperature, as reported in Ma et al. (1990).

\subsubsection{Elimination of $\dot{q}$ in the energy equation}

Substituting for $\dot{q}$ in (4.26) according to (5.14), integrating once, and using the boundary conditions far ahead of the wave, i.e. (5.4), gives (Buchner \& Schneider, $2010 b$ )

$$
\frac{\mathrm{d} \Theta}{\mathrm{d} \eta}=\Theta-\xi+\underbrace{\frac{1}{\sqrt{\pi}} H \lambda \int_{-\infty}^{\eta} \frac{\Theta(s)}{\sqrt{\eta-s}} \mathrm{~d} s}_{\text {heat loss term }}
$$

This integro-differential equation combines with the relevant set of rate equations, i.e. 4.29, 4.31) or 4.34), to form a set of two to five equations. The eigenvalue $\lambda$ has to be determined such that the boundary conditions (5.4) and (5.5) are satisfied.

\subsection{Thermal contact resistance at the interface}

Often, there is no perfect contact between two solids. As a consequence, when heat flows between the two bodies, an apparent temperature drop across the interface is observed (see e.g. Holman (2010)). This temperature drop is related to the heat flux across the interface by

$$
T_{+}-T_{-}=R_{i} \dot{q}_{i}
$$

where $R_{i}$ is the thermal contact resistance of the interface, and the subscripts + and - denote the temperature directly above the interface (i.e. in the layer) and below (i.e. in the substrate), respectively. In dimensionless form, this becomes

$$
\begin{gathered}
\Theta_{+}-\Theta_{-}=R \dot{q}, \\
R:=R_{i} U \rho_{L} c_{p L} \frac{e_{S}}{e_{L}},
\end{gathered}
$$

where $R$ is the dimensionless thermal contact resistance. 
When thermal contact resistance is included, some of the previous equations have to be modified. Equation (5.13) becomes

$$
\Theta_{-}(\eta)=\frac{1}{\sqrt{\pi}} \int_{-\infty}^{\eta} \frac{\dot{q}(s)}{\sqrt{\eta-s}} \mathrm{~d} s .
$$

With (5.17), it follows that Equation (5.13) becomes

$$
\Theta_{+}(\eta)-R \dot{q}(\eta)=\frac{1}{\sqrt{\pi}} \int_{-\infty}^{\eta} \frac{\dot{q}(s)}{\sqrt{\eta-s}} \mathrm{~d} s
$$

Here $\Theta_{+}(\eta)$ is the temperature in the layer, so we can drop the subscript for convenience from now on.

Equation (5.20) is an Abel's integral equation of the second kind. It can be transformed (Polyanin \& Manzhirov, 1999) to

$$
\begin{aligned}
& \dot{q}(\eta)=F(\eta)+\frac{1}{R^{2}} \int_{-\infty}^{\eta} \exp \left[\frac{1}{R^{2}}(\eta-s)\right] F(s) \mathrm{d} s \\
& F(\eta)=\frac{1}{R} \Theta(\eta)-\frac{1}{R^{2} \sqrt{\pi}} \int_{-\infty}^{\eta} \frac{\Theta(s)}{\sqrt{\eta-s}} \mathrm{~d} s .
\end{aligned}
$$

Replacing $F(\eta)$ in (5.21) using (5.22) leads to a double-integral expression which is very complex to solve numerically. Therefore, expansions for small and large values of $R$, respectively, are used to investigate this problem within the existing numerical framework. Both expansions are presented in the following sections.

\subsubsection{Expansion for small thermal contact resistance $\mathbf{R}$}

Assuming a small influence of the thermal contact resistance, i.e. $R \ll 1$, we can expand the non-dimensional temperature as follows:

$$
\begin{aligned}
\Theta(\eta) & =\Theta_{0}(\eta)+R \Theta_{1}(\eta)+\ldots \\
\dot{q}(\eta) & =\dot{q}_{0}(\eta)+R \dot{q}_{1}(\eta)+\ldots
\end{aligned}
$$


Inserting into 5.20 and arranging by orders of $R$ gives:

$$
\begin{aligned}
& R^{0}: \quad \Theta_{0}(\eta)=\frac{1}{\sqrt{\pi}} \int_{-\infty}^{\eta} \frac{\dot{q}_{0}(s)}{\sqrt{\eta-s}} \mathrm{~d} s \\
& R^{1}: \quad \Theta_{1}(\eta)-\dot{q}_{0}(\eta)=\frac{1}{\sqrt{\pi}} \int_{-\infty}^{\eta} \frac{\dot{q}_{1}(s)}{\sqrt{\eta-s}} \mathrm{~d} s .
\end{aligned}
$$

Equation (5.25) is equivalent to $(5.13)$, so the inversion gives (Polyanin \& Manzhirov, 1999):

$$
\dot{q_{0}}(\eta)=\frac{1}{\sqrt{\pi}} \frac{\mathrm{d}}{\mathrm{d} \eta} \int_{-\infty}^{\eta} \frac{\Theta_{0}(s)}{\sqrt{\eta-s}} \mathrm{~d} s=\frac{1}{\sqrt{\pi}} \int_{-\infty}^{\eta} \frac{\Theta_{0}^{\prime}(s)}{\sqrt{\eta-s}} \mathrm{~d} s .
$$

Inserting into (5.26) and then inverting the resulting integral equation yields

$$
\begin{gathered}
\Theta_{1}=\frac{1}{\sqrt{\pi}} \int_{-\infty}^{\eta} \frac{\dot{q}_{1}(s)+\Theta_{0}^{\prime}(s)}{\sqrt{\eta-s}} \mathrm{~d} s \\
\dot{q}_{1}(\eta)+\Theta_{0}^{\prime}(\eta)=\frac{1}{\sqrt{\pi}} \frac{\mathrm{d}}{\mathrm{d} \eta} \int_{-\infty}^{\eta} \frac{\Theta_{1}(s)}{\sqrt{\eta-s}} \mathrm{~d} s
\end{gathered}
$$

where ' denotes the derivative. Inserting (5.27) and (5.29) into (5.24), using $\Theta_{0}^{\prime}=$ $\Theta^{\prime}-R \Theta_{1}^{\prime}-\ldots$ and $(5.23)$, and neglecting terms of $O\left(R^{2}\right)$, we arrive at

$$
\dot{q}(\eta)=\frac{1}{\sqrt{\pi}} \frac{\mathrm{d}}{\mathrm{d} \eta} \int_{-\infty}^{\eta} \frac{\Theta(s)}{\sqrt{\eta-s}} \mathrm{~d} s-R \Theta^{\prime}(\eta)
$$

which is (5.14) with a correction term accounting for a small thermal contact resistance. Substituting $\dot{q}$ in (4.26) and integrating once finally yields

$$
\frac{\mathrm{d} \Theta}{\mathrm{d} \eta}=(1-R H \lambda) \Theta-\xi+\frac{1}{\sqrt{\pi}} H \lambda \int_{-\infty}^{\eta} \frac{\Theta(s)}{\sqrt{\eta-s}} \mathrm{~d} s
$$

which is 5.15 with a correction term accounting for a small thermal contact resistance, accurate up to $O(R)$. It is now convenient to define a scaled dimensionless thermal contact resistance

$$
R^{*}:=R H \lambda=R_{i} \frac{\rho_{S} c_{p S}}{\rho_{L} c_{p L}} \frac{k_{S}}{\delta_{L}}
$$


which, in contrast to $R$, is independent of $U$ and contains only material parameters and the layer thickness. Furthermore, for $H \rightarrow 0$, also $R^{*} \rightarrow 0$, so that in the adiabatic case, $R^{*} \equiv 0$. The final result for the energy equation becomes

$$
\frac{\mathrm{d} \Theta}{\mathrm{d} \eta}=\left(1-R^{*}\right) \Theta-\xi+\frac{1}{\sqrt{\pi}} H \lambda \int_{-\infty}^{\eta} \frac{\Theta(s)}{\sqrt{\eta-s}} \mathrm{~d} s
$$

\subsubsection{Expansion for large thermal contact resistance $\mathbf{R}$}

Equation (5.20) can be rewritten as

$$
\Theta(\eta)=R \dot{q}(\eta)+\frac{1}{R} \frac{1}{\sqrt{\pi}} \int_{-\infty}^{\eta} \frac{R \dot{q}(s)}{\sqrt{\eta-s}} \mathrm{~d} s
$$

with the subscript + dropped as indicated above. Assuming a large influence of the thermal contact resistance, i.e. $R \gg 1, R \dot{q}(\eta)$ must be $O(1)$ like $\Theta$. Therefore, the second term on the right hand side of $(5.34)$ is $O(1 / R) \ll 1$. Equation (5.34) can then be expanded, keeping only leading order terms, to

$$
\Theta(\eta)=R \dot{q}(\eta)+\ldots
$$

Consequently, (4.26) becomes

$$
\frac{\mathrm{d} \Theta}{\mathrm{d} \eta}=\frac{\mathrm{d}^{2} \Theta}{\mathrm{d} \eta^{2}}+\frac{\mathrm{d} \xi}{\mathrm{d} \eta}-\frac{H \lambda}{R} \Theta
$$

Integrating once gives,

$$
\frac{\mathrm{d} \Theta}{\mathrm{d} \eta}=\Theta-\xi+\frac{H \lambda}{R} \int_{-\infty}^{\eta} \Theta(s) \mathrm{d} s
$$

which can be split into two differential equations:

$$
\begin{aligned}
& \frac{\mathrm{d} \Theta}{\mathrm{d} \eta}=\Theta-\xi+\frac{H^{2} \lambda^{2}}{R^{*}} \Omega, \\
& \frac{\mathrm{d} \Omega}{\mathrm{d} \eta}=\Theta .
\end{aligned}
$$

Here, 5.32 has been used to replace $R$ by $R^{*}$. Thus, the integral term is eliminated at the cost of an additional differential equation. It is also remarkable that the non-local influence disappears in this limiting case, and the result is equivalent to 
the approach based on a heat transfer coefficient as often used in the literature, cf. section 5.2.2. 


\section{Precursor region}

Since the temperature far ahead of the wave $T_{S}$ is assumed to be lower than the glass transition temperature $T_{g}$, there is a precursor region that is characterized by vanishing crystallization rate and, consequently, also vanishing degree of crystallization $\xi$. With $\xi \equiv 0$, and disregarding thermal contact resistance for now, 5.15 reduces to

$$
\frac{\mathrm{d} \Theta}{\mathrm{d} \eta}=\Theta+\frac{1}{\sqrt{\pi}} H \lambda \int_{-\infty}^{\eta} \frac{\Theta(s)}{\sqrt{\eta-s}} \mathrm{~d} s
$$

\subsection{Temperature distribution for $R=0$}

The solution of (6.1) can be guessed to be an exponential function. Choosing the origin of the coordinate $\eta$ such that $\eta=0$ for $\Theta=\Theta_{g}$, the solution of (6.1) becomes

$$
\Theta=\Theta_{g} \exp (K \eta) \text { for } \eta \leq 0
$$

where the positive constant $K$ has to satisfy the equation

$$
(K-1) \sqrt{K}=H \lambda
$$

Since $H \lambda$ is defined as a positive parameter, 6.3 has only one real solution for $K$, which must be larger than 1 (Buchner \& Schneider, 2010b):

$$
\begin{aligned}
& K=\frac{1}{3}\left(2+N+\frac{1}{N}\right) \\
& N=\left[\frac{27}{2}(H \lambda)^{2}-1+3 \sqrt{3} H \lambda \sqrt{\frac{27}{4}(H \lambda)^{2}-1}\right]^{1 / 3} .
\end{aligned}
$$

At first glance it may appear that 6.4 represents a real solution only if $(27 / 4)(H \lambda)^{2} \geq 1$. But, for $(27 / 4)(H \lambda)^{2}<1$, the imaginary parts cancel out, so 
that (6.4) gives a real solution for all (positive) values of $H \lambda$. This can be understood if $6.4 \mathrm{a}$ is written as

$$
K=\frac{2}{3}+\frac{1}{3}\left(a+i b+\frac{1}{a+i b}\right)
$$

where $i$ is the imaginary symbol, $a=\operatorname{Re}(N)$ and $b=\operatorname{Im}(N)$. The second term on the right-hand side of 6.5 is real if $a^{2}+b^{2}=1$. This is the case for (6.4).

The dependence of $K$ on $H \lambda$ is shown in fig. 6.1 on page 40, case $R=0$. Of particular interest is the behavior of the solution for small values of $H \lambda$. Expanding (6.3) accordingly gives $K=1+H \lambda+\ldots$ in first order.

\subsection{Heat loss distribution for $R=0$}

The precursor solution for the temperature distribution 6.2 enables us to partition (5.15) even more, since now the integral heat loss term can be split at $\eta=0$. This reduces the integration interval significantly:

$$
\begin{aligned}
\frac{1}{\sqrt{\pi}} H \lambda \int_{-\infty}^{\eta} \frac{\Theta(s)}{\sqrt{\eta-s}} \mathrm{~d} s & =\frac{1}{\sqrt{\pi}} H \lambda\left[\int_{-\infty}^{0} \frac{\Theta_{g} \exp (K s)}{\sqrt{\eta-s}} \mathrm{~d} s+\int_{0}^{\eta} \frac{\Theta(s)}{\sqrt{\eta-s}} \mathrm{~d} s\right] \\
& =H \lambda\left[\frac{\Theta_{g}}{\sqrt{K}} \exp (K \eta) \operatorname{erfc}(\sqrt{K \eta})+\frac{1}{\sqrt{\pi}} \int_{0}^{\eta} \frac{\Theta(s)}{\sqrt{\eta-s}} \mathrm{~d} s\right]
\end{aligned}
$$

where erfc is the complementary error function. Consequently, 5.15 becomes

$$
\frac{\mathrm{d} \Theta}{\mathrm{d} \eta}=\Theta-\xi+H \lambda\left[\frac{\Theta_{g}}{\sqrt{K}} \exp (K \eta) \operatorname{erfc}(\sqrt{K \eta})+\frac{1}{\sqrt{\pi}} \int_{0}^{\eta} \frac{\Theta(s)}{\sqrt{\eta-s}} \mathrm{~d} s\right]
$$

and the problem, including the rate equations, has to be solved on the semi-infinite space, i.e. $\eta>0$. 


\subsection{Influence of thermal contact resistance}

\subsubsection{Small values of $R$}

Including the thermal contact resistance parameter $R^{*}$ (see section 5.3) yields a slightly more complicated formula for $K$. Starting from 5.33 instead of 5.15 yields

$$
\left(K-1+R^{*}\right) \sqrt{K}=H \lambda
$$

instead of 6.3 , and

$$
\begin{aligned}
& K=\frac{1}{3}\left[2\left(1-R^{*}\right)+N+\frac{\left(1-R^{*}\right)^{2}}{N}\right], \\
& N=\left[\frac{27}{2}(H \lambda)^{2}-\left(1-R^{*}\right)^{3}+3 \sqrt{3} H \lambda \sqrt{\frac{27}{4}(H \lambda)^{2}-\left(1-R^{*}\right)^{3}}\right]^{1 / 3}
\end{aligned}
$$

instead of 6.4). Again, it has been confirmed that $K$ remains real for $H, \lambda, R^{*} \geq 0$.

\subsubsection{Large values of $R$}

In case of large thermal contact resistance, plugging 6.2 into 5.36 gives

$$
K=\frac{1}{2}+\sqrt{\frac{1}{4}+\frac{H^{2} \lambda^{2}}{R^{*}}} .
$$

The boundary condition for the differential equation for $\Omega(\eta)$, i.e. (5.38b), is

$$
\Omega(0)=\frac{\Theta_{g}}{K}
$$

Figure 6.1 shows the dependence of $K$ on $H \lambda$ for the various approximations. The curve $R \ll 1$ is similar to the behaviour without thermal contact resistance. In case of $R \gg 1$, the value of $K$ increases much more slowly, showing the influence of the large thermal contact resistance. For $H \lambda \rightarrow 0$, all three relations give the adiabatic value, i.e. $K=1$. 


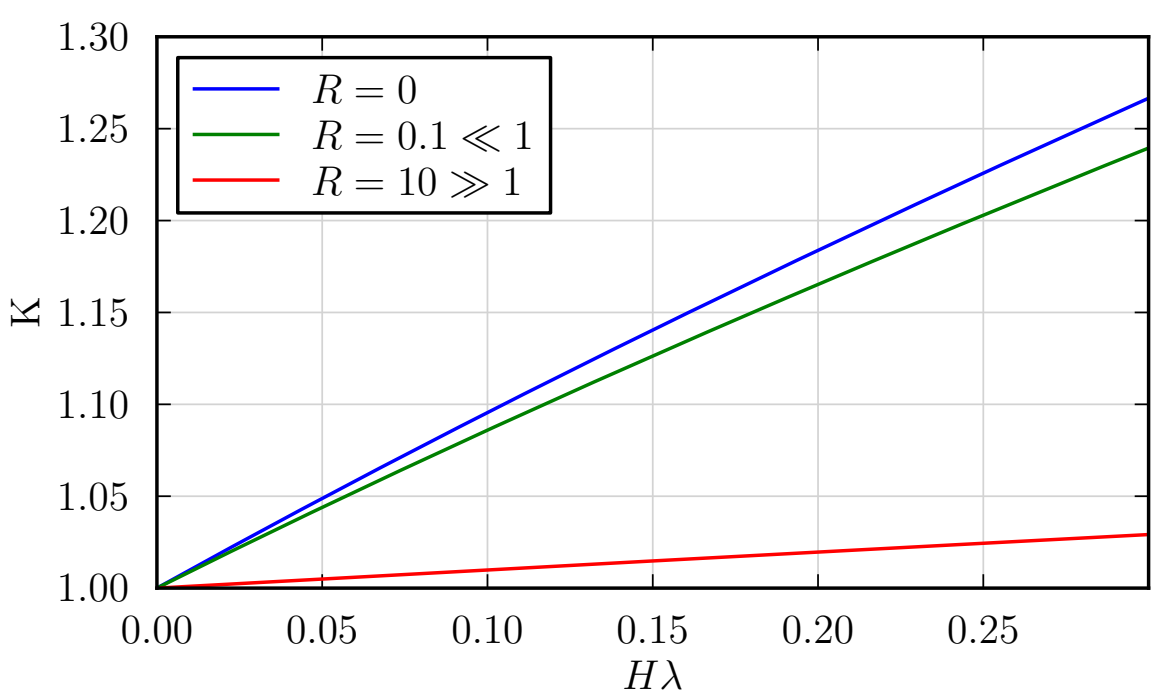

Figure 6.1.: Dependence of the precursor exponent $K$ on the parameter $H \lambda$ for various relations: $R=0:(6.4), R=0.1:$ 6.9), $R=10:(6.10)$. 


\section{Asymptotic behaviour far behind the wave}

It is desirable to obtain an understanding of the behaviour of $\Theta(\eta)$ far behind the crystallization zone, i.e. for $\eta \rightarrow \infty$. In most situations, crystallization can safely assumed to be complete, so $\xi \equiv 1$. $\Theta$ will decrease to the substrate temperature $\Theta=0$ as $\eta \rightarrow \infty$.

\subsection{Case of negligible thermal contact resistance, $\mathrm{R}=0$}

First, the problem formulation without thermal contact resistance is analyzed. Far behind the crystallization wave, $\Theta(\eta)$ is assumed to be of the form

$$
\Theta(\eta \rightarrow \infty)=\frac{C_{\infty}}{\sqrt{\eta}}
$$

To prove that the assumption $(7.1)$ is correct, and to determine $C_{\infty}$, the integral in the energy equation 5.15 is split into three regions:

$$
\frac{\mathrm{d} \Theta}{\mathrm{d} \eta}=\Theta-\xi+\frac{1}{\sqrt{\pi}} H \lambda[\underbrace{\int_{-\infty}^{0} \frac{\Theta(s)}{\sqrt{\eta-s}} \mathrm{~d} s}_{\mathrm{A}}+\underbrace{\int_{0}^{\eta_{C}} \frac{\Theta(s)}{\sqrt{\eta-s}} \mathrm{~d} s}_{\mathrm{B}}+\underbrace{\int_{\eta_{C}}^{\eta} \frac{\Theta(s)}{\sqrt{\eta-s}} \mathrm{~d} s}_{\mathrm{C}}],
$$

with some $\eta_{C} \gg 1$ and $\eta \gg \eta_{C}$. The term A encompasses the precursor region, so $\Theta(s)$ is analytically known, see 6.2). Using the mean value theorem for integration, the term B becomes

$$
\int_{0}^{\eta_{C}} \frac{\Theta(s)}{\sqrt{\eta-s}} \mathrm{~d} s=\Theta\left(\eta_{B}\right) \int_{0}^{\eta_{C}} \frac{1}{\sqrt{\eta-s}} \mathrm{~d} s
$$

where $\eta_{B}$ is unknown, with $0<\eta_{B}<\eta_{C}$. In term $\mathrm{C}, \Theta(s)$ is described by (7.1). 
Consequently, considering a point $\eta>\eta_{C}, 7.2$ becomes

$$
\begin{aligned}
-\frac{1}{2} C_{\infty} \eta^{-3 / 2}=C_{\infty} \eta^{-1 / 2}- & 1+H \lambda\left[\frac{\Theta_{g}}{\sqrt{\pi}} \int_{-\infty}^{0} \frac{\exp (K s)}{\sqrt{\eta-s}} \mathrm{~d} s\right. \\
& \left.+\frac{\Theta\left(\eta_{B}\right)}{\sqrt{\pi}} \int_{0}^{\eta_{C}} \frac{1}{\sqrt{\eta-s}} \mathrm{~d} s+\frac{C_{\infty}}{\sqrt{\pi}} \int_{\eta_{C}}^{\eta} \frac{1}{\sqrt{s} \sqrt{\eta-s}} \mathrm{~d} s\right] .
\end{aligned}
$$

After resolving the integrals, this results in

$$
\begin{aligned}
-\frac{1}{2} C_{\infty} \eta^{-3 / 2}=C_{\infty} \eta^{-1 / 2} & -1+H \lambda\left[\frac{\Theta_{g}}{\sqrt{K}} \exp (K \eta) \operatorname{erfc}(\sqrt{K \eta})\right. \\
& \left.+\frac{2 \Theta\left(\eta_{B}\right)}{\sqrt{\pi}}\left(\sqrt{\eta}-\sqrt{\eta-\eta_{C}}\right)+\frac{2 C_{\infty}}{\sqrt{\pi}} \arccos \left(\sqrt{\frac{\eta_{C}}{\eta}}\right)\right] .
\end{aligned}
$$

For $\eta \rightarrow \infty$ and $\eta \gg \eta_{C}$, the following asymptotic expansions can be used:

$$
\begin{aligned}
\exp (K \eta) \operatorname{erfc}(\sqrt{K \eta}) & =\frac{1}{\sqrt{\pi K \eta}}+O\left(\eta^{-3 / 2}\right) & & \text { as } \eta \rightarrow \infty \\
\arccos \left(\sqrt{\frac{\eta_{C}}{\eta}}\right) & =\frac{\pi}{2}-\sqrt{\frac{\eta_{C}}{\eta}}+O\left[\left(\frac{\eta_{C}}{\eta}\right)^{3 / 2}\right] & & \text { as } \frac{\eta_{C}}{\eta} \rightarrow 0 \\
\sqrt{\eta}-\sqrt{\eta-\eta_{C}} & =\sqrt{\eta}\left\{\frac{1}{2} \frac{\eta_{C}}{\eta}+O\left[\left(\frac{\eta_{C}}{\eta}\right)^{2}\right]\right\} & & \text { as } \frac{\eta_{C}}{\eta} \rightarrow 0
\end{aligned}
$$

Inserting these into 7.5 gives, up to $O\left(\eta^{-1 / 2}\right)$,

$$
C_{\infty} \eta^{-1 / 2}-1+\frac{1}{\sqrt{\pi}} H \lambda\left[\frac{\Theta g}{K} \eta^{-1 / 2}+\Theta\left(\eta_{B}\right) \eta_{C} \eta^{-1 / 2}+\pi C_{\infty}-2 C_{\infty} \sqrt{\eta_{C}} \eta^{-1 / 2}\right]+\ldots=0
$$

Using only the leading order terms results in

$$
C_{\infty}=\frac{1}{\sqrt{\pi} H \lambda}
$$

Considering terms of $O\left(\eta^{-1 / 2}\right)$ yields

$$
1+\frac{\Theta_{g}}{K}(H \lambda)^{2}+\Theta\left(\eta_{B}\right)(H \lambda)^{2} \eta_{C}-\frac{2}{\sqrt{\pi}}(H \lambda) \sqrt{\eta_{C}}=0
$$

With $\Theta_{g}, K$ and $\Theta\left(\eta_{B}\right)$ being $O(1)$, it is necessary that $H \lambda=O\left(1 / \sqrt{\eta_{C}}\right)$. Thus, the previous condition that $\eta_{C} \gg 1$ implies that the present derivation is only valid for $(H \lambda)^{2} \ll 1$. 
Finally, (7.1) becomes

$$
\Theta(\eta)=\frac{1}{H \lambda \sqrt{\pi \eta}} \text { as } \eta \rightarrow+\infty
$$

Thus, the temperature in the fully crystallized layer decays algebraically, i.e. rather slowly in comparison to the exponential increase in the precursor region. Furthermore, this result will be useful for the numerical solution, cf. chapter 9 .

Equation (7.9) may be used to estimate the length of the cooling region behind the crystallization region. Since the change of $\Theta$ is of the order of 1 in the cooling region, the length of the cooling zone is of the order of $\eta=O\left([H \lambda]^{-2}\right)$, i.e. very large for small values of $H \lambda$ (Buchner \& Schneider, 2010b).

\subsection{Influence of thermal contact resistance}

\subsubsection{Small values of $\mathbf{R}$}

The influence of $R^{*}$ on the asymptotic behaviour far behind the wave only appears in the $O\left(\eta^{-1 / 2}\right)$ term, i.e. not in the leading order, and does therefore not change the result of eq. (7.9).

\subsubsection{Large values of $R$}

In case of large thermal contact resistance, the lack of the integral term in the energy equation means we have to choose a different expression for the temperature decay far behind the wave:

$$
\Theta(\eta \rightarrow \infty)=\exp \left(-C_{\infty} \eta\right)
$$

Then it follows from 5.36 that

$$
C_{\infty}=-\frac{1}{2}+\sqrt{\frac{1}{4}+\frac{H^{2} \lambda^{2}}{R^{*}}}
$$

This exponential decay of 7.10 is faster than the previously found algebraic decay (7.9) as $\eta \rightarrow \infty$. 


\section{Stretched coordinate system}

For weakly conducting substrates and small values of $R$ the decay of $\Theta$ as $\eta \rightarrow \infty$ is rather slow. This makes a numerical solution a bit cumbersome. It is therefore convenient to transform the semi-infinite $\eta$-domain, i.e. $\eta=[0, \infty)$, into a finite domain $z=[0,1]$.

\subsection{Coordinate transformation}

Motivated by the asymptotic behavior of the solution for large values of $\eta$, cf. (7.9), the transformation

$$
\begin{aligned}
z & =1-\frac{1}{\sqrt{1+\beta \eta}}, \\
\eta & =\frac{1}{\beta}\left[\frac{1}{(1-z)^{2}}-1\right], \\
\frac{\mathrm{d} z}{\mathrm{~d} \eta} & =\frac{\beta}{2}(1-z)^{3} \\
f(\eta) & =\hat{f}(z),
\end{aligned}
$$

was chosen, where $\beta$ is a stretching parameter, set to the value $\beta=1$, and $f$ stands for any dependent variable.

As a result of the transformation, the asymptotic behaviour of $\hat{\Theta}$, cf. (7.9), is linear in $\mathrm{z}$ :

$$
\hat{\Theta}(z)=\frac{\sqrt{\beta}}{\sqrt{\pi} H \lambda}(1-z) \text { as } z \rightarrow 1 \text {. }
$$

\subsection{Transformed system of equations}

While the coordinate transformation yields the desired finite calculation domain, it has an unfortunate side effect: As will be seen in what follows, the equation system gains a singularity at $z=1$. 


\subsubsection{Energy equation for $R=0$}

Transforming to the new coordinate system, the energy equation (6.7) becomes

$$
\begin{aligned}
\frac{\beta}{2}(1-z)^{3} \frac{\mathrm{d} \hat{\Theta}}{\mathrm{d} z}=\hat{\Theta}-\hat{\xi}+H \lambda & {\left[\frac{\Theta_{g}}{\sqrt{K}} \operatorname{erfcx}\left(\sqrt{\frac{K}{\beta}\left(\frac{1}{(1-z)^{2}}-1\right)}\right)+\right.} \\
& \left.\frac{2}{\sqrt{\pi \beta}}(1-z) \int_{0}^{z} \hat{\Theta}(\bar{z}) \frac{1}{(1-\bar{z})^{2} \sqrt{(1-\bar{z})^{2}-(1-z)^{2}}} \mathrm{~d} \bar{z}\right] .
\end{aligned}
$$

Here the scaled complementary error function $\operatorname{erfcx}(x)=\exp \left(x^{2}\right) \operatorname{erfc}(x)$ has been introduced to improve readability. The relevant boundary conditions are

$$
\begin{aligned}
& \hat{\Theta}(0)=\Theta_{g}, \\
& \hat{\Theta}(1)=0 .
\end{aligned}
$$

\subsubsection{Approximation for small values of $\mathbf{R}$}

In case of the approximation for $R \ll 1,8.3$ becomes

$$
\begin{aligned}
\frac{\beta}{2}(1-z)^{3} \frac{\mathrm{d} \hat{\Theta}}{\mathrm{d} z}=(1- & \left.R^{*}\right) \hat{\Theta}-\hat{\xi}+H \lambda\left[\frac{\Theta_{g}}{\sqrt{K}} \operatorname{erfcx}\left(\sqrt{\frac{K}{\beta}\left(\frac{1}{(1-z)^{2}}-1\right)}\right)+\right. \\
& \left.\frac{2}{\sqrt{\pi \beta}}(1-z) \int_{0}^{z} \hat{\Theta}(\bar{z}) \frac{1}{(1-\bar{z})^{2} \sqrt{(1-\bar{z})^{2}-(1-z)^{2}}} \mathrm{~d} \bar{z}\right],
\end{aligned}
$$

with unchanged boundary conditions (8.4).

\subsubsection{Approximation for large values of $\mathbf{R}$}

In case of the approximation for $R \gg 1,5.38$ becomes

$$
\begin{aligned}
& \frac{\beta}{2}(1-z)^{3} \frac{\mathrm{d} \hat{\Theta}}{\mathrm{d} z}=\hat{\Theta}-\hat{\xi}+\frac{H^{2} \lambda^{2}}{R^{*}} \hat{\Omega}, \\
& \frac{\beta}{2}(1-z)^{3} \frac{\mathrm{d} \hat{\Omega}}{\mathrm{d} z}=\hat{\Theta}
\end{aligned}
$$

with unchanged boundary conditions 8.4 and additionally (6.11) for $\hat{\Omega}$. 


\subsubsection{Rate equations}

In the rate equations, only the derivative term is affected. In case of heterogeneous crystallization, 4.29) becomes

$$
\begin{aligned}
\frac{\beta}{2}(1-z)^{3} \frac{\mathrm{d} \hat{\xi}}{\mathrm{d} z} & =\lambda^{2} G(\hat{\Theta}) \hat{\varphi}_{1}(z)(1-\hat{\xi}) \\
\frac{\beta}{2}(1-z)^{3} \frac{\mathrm{d} \hat{\varphi}_{1}}{\mathrm{~d} z} & =\lambda^{2} G(\hat{\Theta}) \hat{\varphi}_{2}(z) \\
\frac{\beta}{2}(1-z)^{3} \frac{\mathrm{d} \hat{\varphi}_{2}}{\mathrm{~d} z} & =\lambda^{2} G(\hat{\Theta}) \hat{\varphi}_{3}(z) \\
\frac{\beta}{2}(1-z)^{3} \frac{\mathrm{d} \hat{\varphi}_{3}}{\mathrm{~d} z} & =\lambda^{2} \mu n(\hat{\Theta})\left[1-\hat{\varphi}_{3}(z)\right],
\end{aligned}
$$

with the boundary conditions

$$
\hat{\xi}(0)=\hat{\varphi}_{1}(0)=\hat{\varphi}_{2}(0)=\hat{\varphi}_{3}(0)=0 .
$$

If using a single rate equation, 4.31 becomes

$$
\frac{\beta}{2}(1-z)^{3} \frac{\mathrm{d} \hat{\xi}}{\mathrm{d} z}=\lambda^{2} G(\hat{\Theta}) g(\hat{\xi}),
$$

with the boundary condition

$$
\hat{\xi}(0)=0 .
$$

In case of homogeneous crystallization, 4.34 becomes

$$
\begin{aligned}
\frac{\beta}{2}(1-z)^{3} \frac{\mathrm{d} \hat{\xi}}{\mathrm{d} z} & =\lambda^{2} G(\hat{\Theta}) \hat{\phi}_{1}(z)(1-\hat{\xi}) \\
\frac{\beta}{2}(1-z)^{3} \frac{\mathrm{d} \hat{\phi_{1}}}{\mathrm{~d} z} & =\lambda^{2} G(\hat{\Theta}) \hat{\phi}_{2}(z) \\
\frac{\beta}{2}(1-z)^{3} \frac{\mathrm{d} \hat{\phi}_{2}}{\mathrm{~d} z} & =\lambda^{2} G(\hat{\Theta}) \hat{\phi}_{3}(z) \\
\frac{\beta}{2}(1-z)^{3} \frac{\mathrm{d} \hat{\phi}_{3}}{\mathrm{~d} z} & =\lambda^{2} M I(\hat{\Theta}),
\end{aligned}
$$

with the boundary conditions

$$
\hat{\xi}(0)=\hat{\phi}_{1}(0)=\hat{\phi}_{2}(0)=\hat{\phi}_{3}(0)=0 \text {. }
$$




\section{Numerical method of solution}

In this chapter, the employed numerical method of solution is explained. The exposition is based on the homogeneous crystallization model with 4 rate equations, but the process is comparable for the other crystallization and thermal contact resistance models presented.

The set of equations solved is the energy equation 8.3 with the boundary conditions 8.4 and the set of rate equations 8.11) with the boundary conditions 8.12.

This problem is solved with bvpsuite (Kitzhofer et al., 2010), a MatLAB code using a collocation method for solving singular boundary value problems in ordinary differential equations. bvpsuite can solve a singularity of the form $1 /(1-z)^{b}$, with $b>1$, which occurs at $z=1$.

A MATLAB program was written to handle the whole computation procedure, from setting problem parameters and configuration settings, to initialisation, computation, postprocessing, storage of results and batch computation.

\subsection{Overview of solution procedure}

A flow chart sketching the solution process is shown in fig. 9.1.

As a first step, all relevant parameters are read from a configuration file. Then, a mesh is generated on the computation domain. Also, a starting guess for $\lambda, \hat{\Theta}(z)$ and $\hat{\xi}(z)$ is computed and plotted. Afterwards, the program flow depends on the nature of the problem:

In the adiabatic case, the process is rather straightforward, and bvpsuite can be directly used to solve the problem.

When including heat loss, it is necessary to deal with the heat loss term in (8.3), i.e. the term beginning with $H$, in an iterative fashion, as detailed in section 9.3. When considering the approximation for $R \ll 1$, eq. (8.5) is solved in the same fashion. In case of $R \gg 1$, the solution method is equivalent to the adiabatic case, with one additional differential equation, cf. (8.6). 


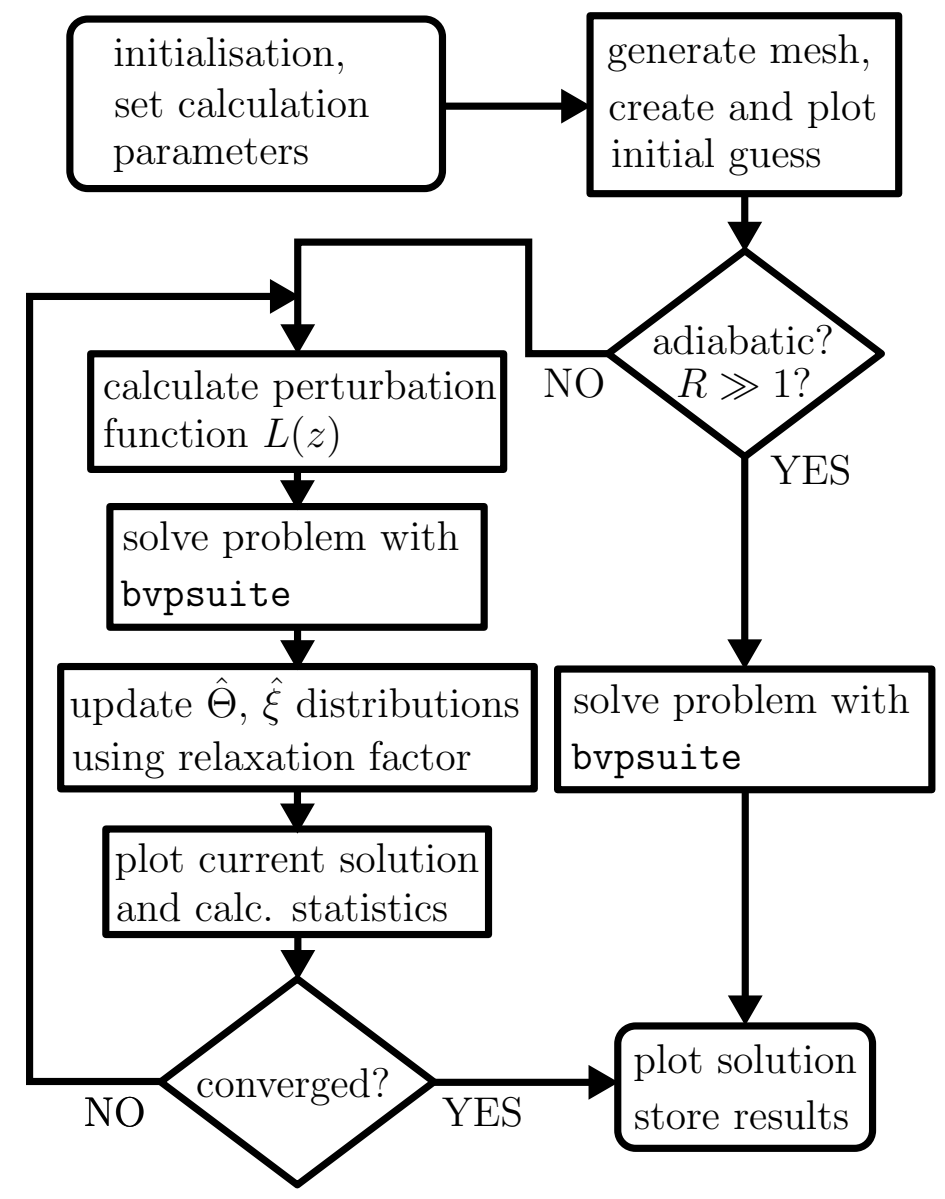

Figure 9.1.: Flowchart showing the program flow in the MATLAB code.

After arriving at a solution, it is plotted, and relevant data are stored automatically. Additionally, it is possible to vary a parameter, and batch-process the computations, including continuation, i.e. using an available solution as a starting guess for a new computation.

Computations were done on a regular Linux PC with an Athlon64 X2 6000+ dualcore processor and 6 GB RAM, using MATLAB R2011a. The computation duration for an adiabatic case was on the order of 90 seconds. For a computation including heat loss, durations were on the order of 30-60 minutes.

\subsection{Mesh generation}

bvpsuite offers the option to perform automatic mesh adaptation to improve the solution accuracy. This is useful in the adiabatic case, and typically results in a mesh 
similar to the one presented in Kitzhofer et al. (2010) for a simplified version of the problem treated here.

In the case involving heat loss, however, automatic mesh adaptation is impractical. Due to the iterative nature of the solution process and the non-exact boundary condition (see section 9.4), an ever-increasing amount of mesh points is allocated near the downstream domain boundary, and the computation ultimately fails. Therefore, a manually adapted mesh is used, which is based on a homogeneous mesh, with cell size refinements around the expected crystallization zone, and near $z=z_{r}$. Results obtained from an adiabatic solution have been used as a guide for the shape of the mesh density distribution, and a resulting cell size distribution can be seen at the bottom of fig. 11.1, page 64 .

\subsection{Iteration procedure}

\subsubsection{Treatment of the integral term}

The integral term makes the energy equation an integro-differential equation, which cannot be solved by bvpsuite. Therefore, the whole integral term is brought into the equation system as a perturbation function $L$, defined as

$$
\begin{aligned}
L(z, \hat{\Theta}(z), \lambda):=H \lambda & {[\overbrace{\frac{\Theta_{g}}{\sqrt{K}} \operatorname{erfcx}\left(\sqrt{\frac{K}{\beta}\left(\frac{1}{(1-z)^{2}}-1\right)}\right)}^{\text {precursor term }}+} \\
& \underbrace{\frac{2}{\sqrt{\pi \beta}}(1-z) \int_{0}^{z} \hat{\Theta}(\bar{z}) \frac{1}{(1-\bar{z})^{2} \sqrt{(1-\bar{z})^{2}-(1-z)^{2}}} \mathrm{~d} \bar{z}}_{\text {integral term }}],
\end{aligned}
$$

where the dependency on fixed problem parameters is not explicitly cited on the left-hand side.

Then, the values for $\hat{\Theta}(z)$ and $\lambda$ are assumed fixed during one iteration step. Therefore, the perturbation function becomes a function only of the space coordinate, $L(z)$. The resulting problem is a system of ordinary differential equations, which can be solved with bvpsuite. 
To improve stability and avoid oscillating divergence of the iteration procedure, it turned out to be necessary to use a relaxation factor to combine the results for $\hat{\Theta}(z)$, $\hat{\xi}(z)$ and $\lambda$ of the previous and current iterations. The iteration starts with a small relaxation factor, e.g. 0.05, and linearly ramps up to a value $<0.5$ after a couple of iterations.

\subsubsection{Iteration methods for integral term}

At the beginning of the new iteration, the new values for $\hat{\Theta}(z)$ and $\lambda$ are used to update $L(z, \hat{\Theta}(z), \lambda)$, yielding an improved $L(z)$. This is the default method for determining $L(z)$, henceforth called iter1.

Near the critical value for $H$ (cf. section 11.3, fig. 11.9), convergence problems are observed. The rate of convergence is severely reduced, or the computations fail to converge at all. A partial solution to this problem is an alternative method of determining the improved $L(z)$, henceforth denoted iter2. In this method, not only $H$, but also $\lambda$ is held at a fixed value in $L(z, \hat{\Theta}(z), \lambda)$. This has the advantage that there is no maximum value for $H \lambda$, as there is for $H$ (see fig. 11.8). Convergence can be achieved in a wider parameter range, enabling a computation beyond the maximum $H$ value, onto the lower branch of fig. 11.9 .

It was confirmed that iter2 gives the same results as iter1. Unfortunately, the permissible step size when varying a fixed $H \lambda$ is much reduced, leading to a greater number of necessary computations to cover the same parameter range as iter 1. This gets worse the farther the computation progresses on the lower branch, making further computations unfeasible beyond a certain point. Therefore, it is advisable to use iter1 as long as possible, and only then switch to iter2.

The numerical computation process for $L(z, \hat{\Theta}(z), \lambda)$ is explained in more detail in section 9.5 .

\subsection{Asymptotic boundary condition near $z=1$}

The code bvpsuite is designed to solve differential equation systems including singularities of the second kind (Kitzhofer et al., 2010), like the one occuring in the present problem at $z=1$.

In the adiabatic case, the problem can therefore typically be solved on the whole spatial domain $z \in[0,1]$. 
For problems involving heat loss, it was not possible to get converged solutions for $z \in[0,1]$. This is presumably due to the simple nature of the iteration procedure, which does not consider the singular nature of the underlying problem.

An asymptotic expression for $\hat{\Theta}(z \rightarrow 1)$ is available, of $(8.2)$, so it is possible to prescribe an approximate value for $\hat{\Theta}$ at a new right boundary $z_{r}<1$. Thus, it is possible to obtain solutions on the domain $z \in\left[0, z_{r}\right]$, with a typical value of $z_{r} \approx 0.99$.

\subsection{Computation of the heat loss term}

The precursor term in (9.1) is straightforward to compute. Using the scaled complementary error function erfcx, which is available in MATLAB, one avoids numerical problems when evaluating $\exp \left(x^{2}\right) \operatorname{erfc}(x)$ for very large values of $x$.

The integral term is a bit more challenging. Initially, the trapezoidal rule was used to compute the integral term from given $\hat{\Theta}$ values. It was found that this approach leads to problems near $\mathrm{z}=1$. Therefore, an alternative routine was implemented which uses an adaptive quadrature based on a Gauss-Kronrod pair, utilizing the MATLAB function quadgk.

To assess the accuracy of both methods, the following analytically integrable test function $\hat{\Theta}_{t}$ was chosen, which exhibits the most important characteristics of the expected temperature distribution, i.e. boundedness on $z \in\left[0, z_{r}\right]$, and correct asymptotic behaviour according to $(8.2)$ :

$$
\hat{\Theta}_{t}(z)=C_{\infty} \arctan (\sqrt{\beta}(1-z)) .
$$

Figure 9.2 shows a plot of $\hat{\Theta}_{t}(z)$. The integral term in $(9.1)$ was evaluated in MATHEMATICA with $(9.2)$ as $\hat{\Theta}(\bar{z})$. The analytical result of the integration is quite lengthy, and not given here. In fig. 9.2, the analytical integration result coincides with the quadgk curve.

A comparison of numerical integration using the trapezoidal rule and quadgk, respectively, on a mesh of 100 equidistant points and $C_{\infty}=\beta=1$ is shown in figs. 9.2 and 9.3 . Clearly, the trapezoidal method has problems near $z=1$, and quadgk is superior, however both methods fail when $z_{r}=1$. The asymptotic behaviour of the integral, i.e. linear in $z$, is independent of the actual temperature distribution $\hat{\Theta}(\bar{z})$, as long as that function's asymptotic behaviour is identical to 8.2 . Therefore, it 


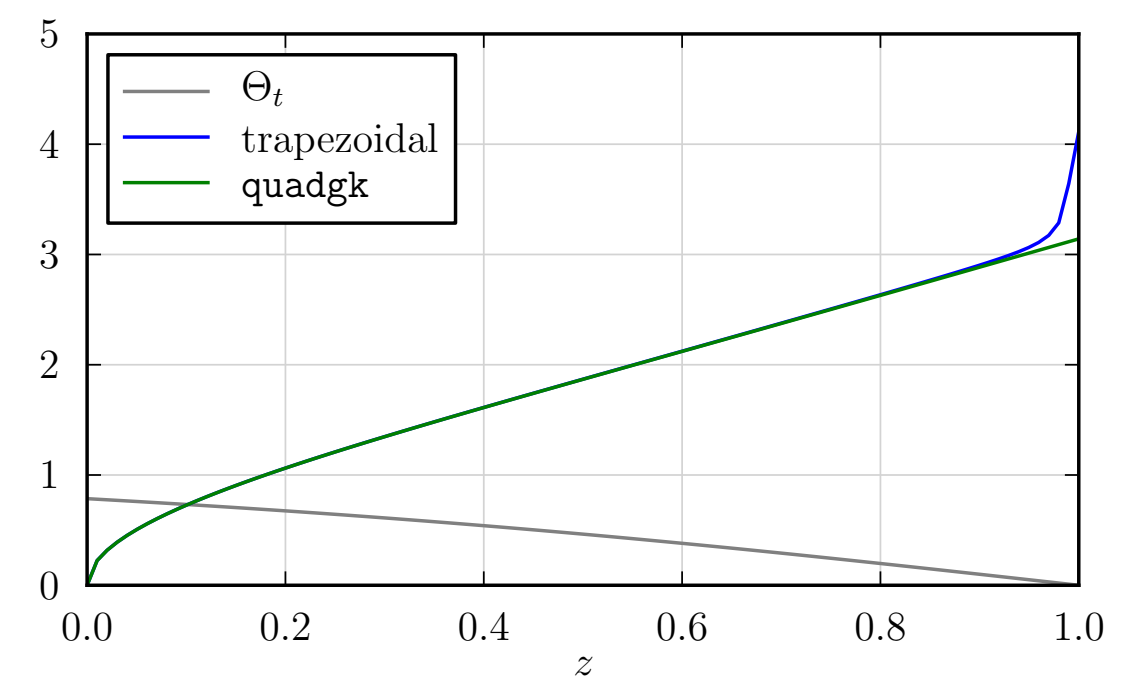

Figure 9.2.: Test of numerical integration methods. Grey: Analytically integrable test function $\hat{\Theta}_{t}(z)$. Blue and green: Results for the trapezoidal and quadgk methods, respectively. The curve for the analytical integration coincides with the quadgk curve.

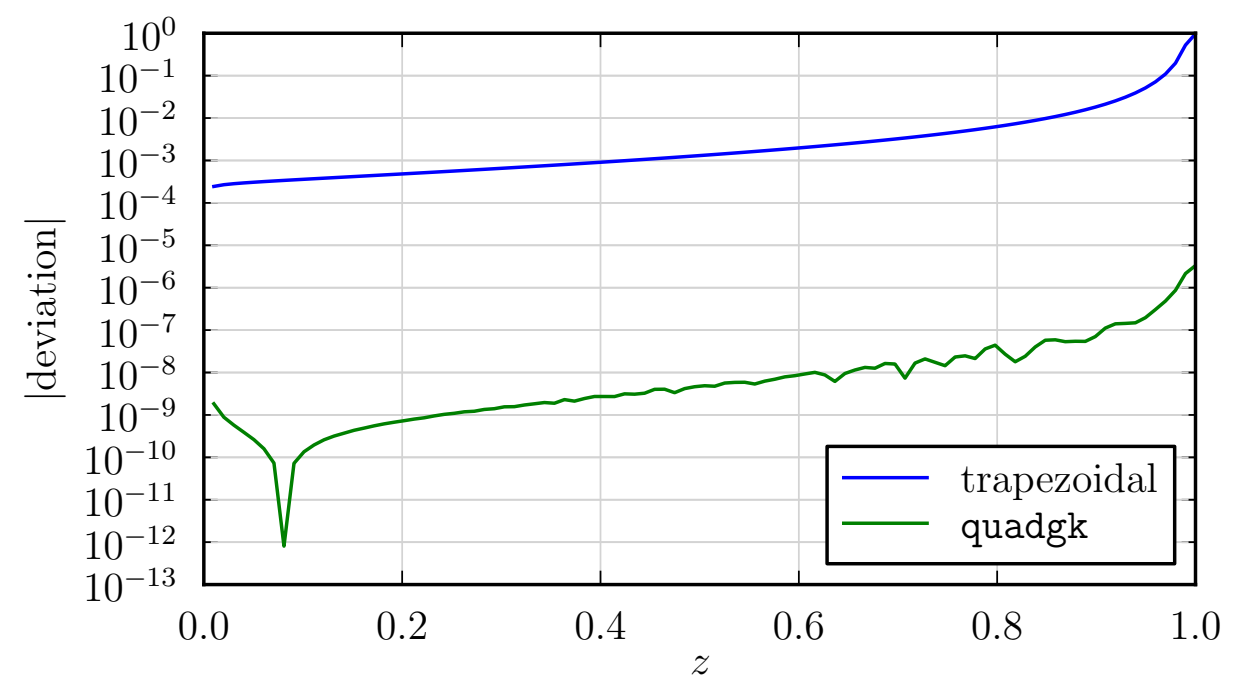

Figure 9.3.: Absolute deviations from analytical integration result for trapezoidal and quadgk numerical integration methods, using the temperature distribution (9.2). quadgk is far more accurate. The spike at $z \approx 0.1$ is due to a sign change in the deviation. 
is feasible to replace the inaccurate value for $z=1$ by an extrapolation from the preceding values.

As an alternative approach to integrating the heat loss term, a numerical integration technique presented in Scheichl et al. (2008) could possibly be adapted for a non-equidistant mesh. 


\section{Parameter determination}

In this chapter, all the necessary parameter values to compute solutions are collected. Often, key parameters necessary for our models are missing or not given in an experimental paper, and have to be obtained from other sources.

Explosive crystallization in germanium has been selected for experimental comparison, because for explosive crystallization in germanium, a direct amorphouscrystalline transition has been observed experimentally (Grigoropoulos et al., 2006).

When considering explosive crystallization in silicon, the following additional articles are of relevance, containing data pertaining to the crystallization process (Farjas \& Roura, 2008; Gall et al., 2010; Kokorowski et al., 1982a; Roura et al., 2009; Schoenfeld et al., 1994, Spaepen \& Turnbull, 1982) or thermodynamic properties of silicon (Donovan et al., 1989; Kumomi \& Yonehara, 1994; Poate, 1983; Shanks et al., 1963).

\subsection{Germanium}

\subsubsection{Parameter sets}

To facilitate comparisons, and for easier designation, three parameter sets for the computation of the following results have been defined, as shown in table 10.1. More detailed information about the respective parameters listed in table 10.1 is given in the following sections.

Parameter sets A and B are for germanium with homogeneous crystallization, where A uses a relation according to (3.17) for crystal growth velocity and nucleation rate, while $\mathrm{B}$ uses 3.18 ) and 3.21 , respectively.

The parameter set $\mathrm{C}$ is used for comparison with experimental results from Grigoropoulos et al. (2006), see section 11.6. 
Table 10.1.: Parameter sets

\begin{tabular}{|c|c|c|c|c|}
\hline Parameter & A & B & C & Unit \\
\hline$\rho_{L}$ & \multicolumn{3}{|c|}{5300} & $\mathrm{~kg} / \mathrm{m}^{3}$ \\
\hline$T_{m}$ & \multicolumn{3}{|c|}{1210} & $\mathrm{~K}$ \\
\hline$l$ & \multicolumn{2}{|c|}{177} & 189 & $\mathrm{~kJ} / \mathrm{kg}$ \\
\hline$c_{p L}$ & \multicolumn{2}{|r|}{377} & 396 & $\mathrm{~J} / \mathrm{kg} \mathrm{K}$ \\
\hline$\alpha_{L}$ & \multicolumn{3}{|c|}{$7.3 \times 10^{-6}$} & $\mathrm{~m}^{2} / \mathrm{s}$ \\
\hline$k_{L}$ & \multicolumn{3}{|c|}{14.6} & $\mathrm{~W} / \mathrm{m} \mathrm{K}$ \\
\hline$V_{\infty}$ & \multicolumn{3}{|c|}{1} & - \\
\hline$G_{0}$ & $3.05 \times 10^{7}$ & $3.20 \times 10^{7}$ & $1 \times 10^{12}$ & $\mathrm{~m} / \mathrm{s}$ \\
\hline$E_{G}$ & 2.16 & 2.155 & 2.3 & $\mathrm{eV}$ \\
\hline$E_{G m}$ & 0 & 0.005 & 0.2 & $\mathrm{eV}$ \\
\hline$I_{0}$ & $8.3 \times 10^{40}$ & $8.5423 \times 10^{40}$ & $1.2 \times 10^{59}$ & $/ \mathrm{m}^{3} \mathrm{~s}$ \\
\hline$E_{I}$ & 1.8 & 1.797 & 3.65 & $\mathrm{eV}$ \\
\hline$E_{I m}$ & 0 & 0.003 & 0.35 & $\mathrm{eV}$ \\
\hline$\rho_{S} c_{p S}$ & \multicolumn{3}{|c|}{$2.31 \times 10^{6}$} & $\mathrm{~J} / \mathrm{m}^{3} \mathrm{~K}$ \\
\hline$\alpha_{S}$ & \multicolumn{3}{|c|}{$1.2 \times 10^{-6}$} & $\mathrm{~m}^{2} / \mathrm{s}$ \\
\hline$\delta_{L}$ & \multicolumn{3}{|c|}{1.8} & $\mu \mathrm{m}$ \\
\hline$T_{S}$ & & 50 & 700 & $\mathrm{~K}$ \\
\hline$T_{g}$ & \multicolumn{3}{|c|}{$T_{S}+10$} & $\mathrm{~K}$ \\
\hline
\end{tabular}

\subsubsection{Maximum crystalline volume fraction}

Due to the complete crystallization of germanium (in contrast to, e.g., polymers), $V_{\infty}=1$ is assumed for the maximum volume fraction of crystalline phase.

\subsubsection{Mass density}

The density of amorphous Ge is reported to be $4600-5900 \mathrm{~kg} / \mathrm{m}^{3}$ Koba \& Wickersham, 1982; Shklovskij \& Kuz'menko, 1989). Because the density of amorphous Ge is close to the density of the crystalline phase (Donovan et al., 1985), a single density value of $\rho_{L}=5300 \mathrm{~kg} / \mathrm{m}^{3}$ is used in the present computations.

For the purposes of conversion between mole-based values and specific values, the standard atomic weight of $72.64 \mathrm{~g} / \mathrm{mol}$ is used (Linstrom \& Mallard, 2005).

\subsubsection{Glass transition temperature}

Due to the chosen crystal growth velocity formulation (3.18), the physical glass transition temperature does not enter the model at all, cf. section $3.3 . T_{g}$ is only to be 
understood as an artificial cut-off temperature greater than $T_{S}$, which is necessary to be able to treat the problem as a propagating wave of invariant shape. This cut-off temperature is set to $T_{g}=T_{S}+10 \mathrm{~K}$.

\subsubsection{Melting temperature}

The melting temperature of the crystalline phase of germanium is given as $T_{m}=$ $1210 \mathrm{~K}$ by many authors, e.g. Donovan et al. (1985, 1983); Grigoropoulos et al. (2006); Koba \& Wickersham (1982); Nikolova et al. (2010); Rogers et al. (2006).

Often, a "melting temperature of the amorphous phase" is given by various authors. The value given for germanium is 960-970 K (Donovan et al., 1985; Grigoropoulos et al., 2006; Koba \& Wickersham, 1982; Rogers et al., 2006). It is also often assumed by authors that this melting "transition" from amorphous to liquid state is endothermic and consequently has an associated latent heat, e.g. in Donovan et al. (1983); Sharma et al. (1984). This seems to imply that, when creating the amorphous material, a first-order phase transition from the liquid to the amorphous state takes place.

This view is not shared by the present author and others, e.g. (Berthier \& Biroli, 2011; Kokorowski et al., 1982b; Marfaing \& Marine, 1995; Olson \& Roth, 1988). In the present work, neither a "melting temperature of the amorphous phase" nor a latent heat associated with a transition at this temperature are considered.

\subsubsection{Latent heat of melting}

The present work is limited to considering a direct transition from the amorphous to the crystalline phase, with an associated specific latent heat. The latent heat of the amorphous-crystalline transition $l$ is reported by a variety of sources, and given in table 10.2 as specific values. Where appropriate, it has been calculated as the difference between the latent heats given for the melt-crystalline and melt-amorphous transitions, respectively. The wide spread of the values of Koba \& Wickersham (1982) includes values for sputtered and evaporated germanium, hinting at an influence of the preparation mechanism on $l$. Furthermore, relaxation processes in amorphous materials can also contribute to influencing the latent heat of transformation (Donovan et al., 1985; Marfaing \& Marine, 1995; Roura \& Farjas, 2009). 
Averaging the values in table 10.2 , a value of $l=177 \mathrm{~kJ} / \mathrm{kg}$ is chosen for the parameter sets A and B. For experimental comparison, the value of $189 \mathrm{~kJ} / \mathrm{kg}$ from Grigoropoulos et al. (2006) is chosen (parameter set C).

Table 10.2.: Specific latent heat of germanium.

\begin{tabular}{|c|c|}
\hline specific latent heat $l[\mathrm{~kJ} / \mathrm{kg}]$ & source \\
\hline $160.0 \pm 9.6$ & Donovan et al. $(1985,1983)$ \\
\hline 167 & Kurtze et al. $(1984)$ \\
\hline $150-300$ & Koba \& Wickersham (1982) \\
\hline 167 & Fan $(1981)$ \\
\hline 155 & Sharma et al. (1984) \\
\hline 189 & Grigoropoulos et al. $(2006)$ \\
\hline
\end{tabular}

\subsubsection{Isobaric specific heat capacity}

Kurtze et al. (1984) give a value for the isobaric specific heat capacity of crystalline germanium of $c_{p L, c}=377 \mathrm{~J} / \mathrm{kg} \mathrm{K}$. This falls near the range given in Koba \& Wickersham (1982), 290-372 J/kg K. $c_{p L, c}=377 \mathrm{~J} / \mathrm{kg} \mathrm{K}$ was used for parameter sets A and $\mathrm{B}$.

For the comparison with experiments, data from Grigoropoulos et al. (2006), $c_{p L, c}=396 \mathrm{~J} / \mathrm{kg} \mathrm{K}$, was used (parameter set C).

\subsubsection{Thermal diffusivity}

The thermal diffusivity is given as $6.32 \times 10^{-6} \mathrm{~m}^{2} / \mathrm{s}$ for amorphous Ge and $8.28 \times 10^{-6}$ $\mathrm{m}^{2} / \mathrm{s}$ for crystalline Ge (Grigoropoulos et al. 2006). Therefore, it is a reasonable approximation to use the average value for $\alpha_{L}=7.3 \times 10^{-6} \mathrm{~m}^{2} / \mathrm{s}$.

\subsubsection{Thermal conductivity}

Grigoropoulos et al. (2006) report the values

$$
\begin{aligned}
k_{a} & =25.15 \mathrm{~W} / \mathrm{m} \mathrm{K}, \\
k_{c} & =17.40 \mathrm{~W} / \mathrm{m} \mathrm{K}, \\
k_{m} & =49.43 \mathrm{~W} / \mathrm{m} \mathrm{K}
\end{aligned}
$$


for germanium. The arithmetic mean of $k_{a}$ and $k_{c}$ is $k_{L}=21.28 \mathrm{~W} / \mathrm{m} \mathrm{K}$. Calculating $k_{L}$ instead by using $\alpha_{L}$ from above, and $\rho_{L} c_{p L}=2.73 \times 10^{6} \mathrm{~J} / \mathrm{m}^{3} \mathrm{~K}$, both from Grigoropoulos et al. (2006), $k_{L}=19.9 \mathrm{~W} / \mathrm{m} \mathrm{K}$ is obtained, a slightly different value.

For consistency, it makes sense to calculate the thermal conductivity from the already specified values for $\alpha_{L}, \rho_{L}$ and $c_{p L}$, which results in $k_{L}=14.6 \mathrm{~W} / \mathrm{m} \mathrm{K}$. A difficulty in uniting all the values from different publications becomes apparent here. It can at least partly be attributed to differing preparation methods for the experiments, as is also illustrated in the wide range of values for $l$ in table 10.2 .

\subsubsection{Crystal growth velocity}

Equation (3.18) is chosen as a suitable crystal growth velocity formulation. As detailed in section 3.3 , often experimental measurements for the crystal growth velocity $G_{C}$ only offer values for $E_{G}$ and $G_{0}$. Relevant values for germanium are available from Claverie et al. (2010); Donovan et al. (1985); Johnson et al. (2008).

Claverie et al. observe that values for $E_{G}$ generally agree well, but values for $G_{0}$ differ by up to two orders of magnitude in the literature! They remark that the highly variable values for $G_{0}$ are possibly rooted in slight systematic errors in the difficult temperature measurements involved in determining $E_{G}$ and $G_{0}$. Indeed, after allowing small errors in the temperature measurements, they manage to successfully align all the growth rate measurements of 7 experiments they consider, arriving at the values $G_{0}=3.05 \times 10^{7} \mathrm{~m} / \mathrm{s}$ and $E_{G}=2.16 \mathrm{eV}$. Additionally, $E_{G m}=0$ (parameter set $\mathrm{A})$.

Using eq. 3.19), one can find an alternative set of parameters such that the experimental measurements are reproduced, but the result is still a bell-shaped curve as in fig. 4.1. Taking into account the maximum temperature of experimental data available in Claverie et al. (2010), $T \approx 800 \mathrm{~K}$, the parameter set $G_{0}=3.20 \times 10^{7} \mathrm{~m} / \mathrm{s}$, $E_{G}=2.155 \mathrm{eV}$ and $E_{G m}=0.005 \mathrm{eV}$ results in less than $10 \%$ deviation from the previous curve at $T=800 \mathrm{~K}$ (parameter set B).

An additional set of parameters has been defined which stems from calculations available for the crystal nucleation rate (cf. parameter set $\mathrm{C}$ in the following section). It preserves the low-temperature behaviour of $G_{C}(T)$ as above, but makes sure the temperature of maximal $G_{C}$ is identical to the temperature of maximal $I_{C}$. This results in $G_{0}=1 \times 10^{12} \mathrm{~m} / \mathrm{s}, E_{G}=2.3 \mathrm{eV}$ and $E_{G m}=0.2 \mathrm{eV}$ (parameter set C).

Figure 10.1 shows a comparison of the crystal growth velocity using the above parameter sets $\mathrm{A}, \mathrm{B}$ and $\mathrm{C}$. 


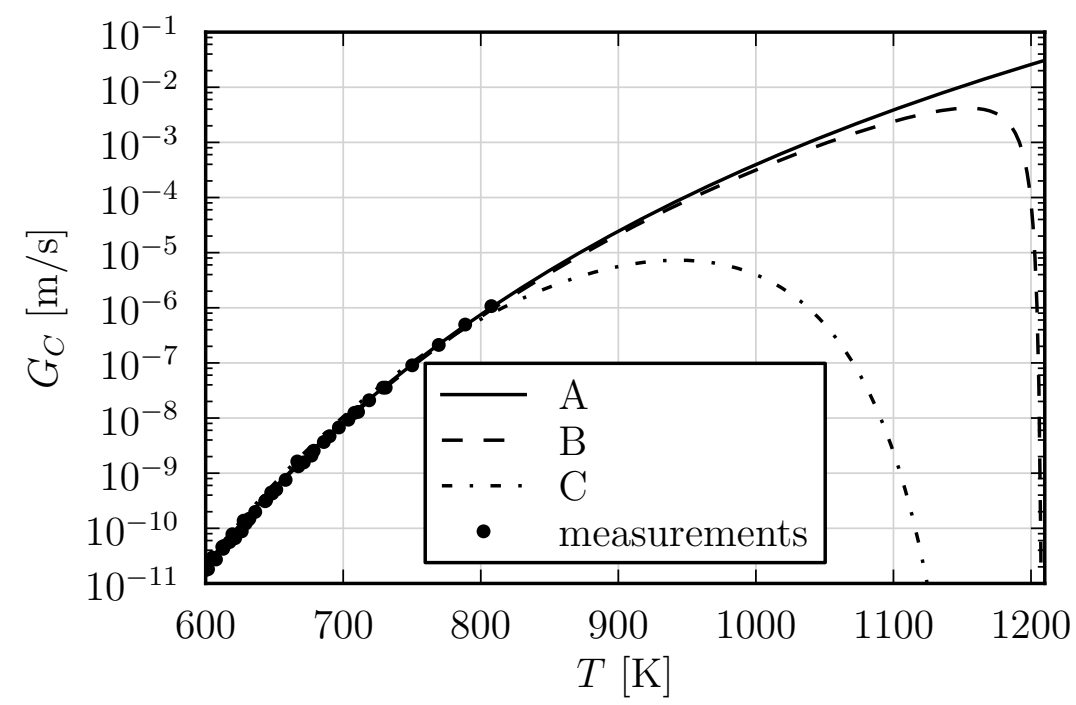

Figure 10.1.: Crystal growth velocity for parameter sets A, B and C (Table 10.1). •: Experimental data from Claverie et al. (2010).

\subsubsection{Crystal nucleation rate}

Following the chosen formulation for the crystal growth velocity, equation (3.21) is chosen for the crystal nucleation rate. Data for the solid-state nucleation in amorphous germanium is available in (Marine \& Marfaing, 1991). As usual in the literature on experiments, an expression of the form (3.17) is used, so for the parameters in (3.18) the values $I_{0}=8.3 \times 10^{40} / \mathrm{m}^{3} \mathrm{~s}, E_{I}=1.8 \pm 0.1 \mathrm{eV}$ and $E_{I m}=0$ are obtained (parameter set A).

In analogy to the crystal growth velocity, one can also find an alternative parameter set for the crystal nucleation rate. Experimental data up to $T \approx 950 \mathrm{~K}$ is available in Marine \& Marfaing (1991). Using a maximum deviation of $10 \%$ for $I_{C}(T)$ at this temperature, like for $G_{C}(T)$ in the previous section, one arrives at $I_{0}=8.5423 \times 10^{40}$ $/ \mathrm{m}^{3} \mathrm{~s}, E_{I}=1.797 \pm 0.100 \mathrm{eV}$ and $E_{I m}=0.003 \mathrm{eV}$ (parameter set B).

In addition to the experimental values, Marine \& Marfaing also show a calculated crystal nucleation rate with a much broader peak than what is obtained using B. This crystal nucleation rate distribution has been reproduced as accurately as possible using (3.21), resulting in $I_{0}=1.2 \times 10^{59} / \mathrm{m}^{3} \mathrm{~s}, E_{I}=3.65 \pm 0.10 \mathrm{eV}$ and $E_{\text {Im }}=0.35$ $\mathrm{eV}$ (parameter set $\mathrm{C}$ ). The plot exhibits a peak in the crystal nucleation rate which is shifted to a much lower temperature of approximately 930-950 K. Interestingly, this temperature is approximately the temperature often cited (Donovan et al., 1985 . 


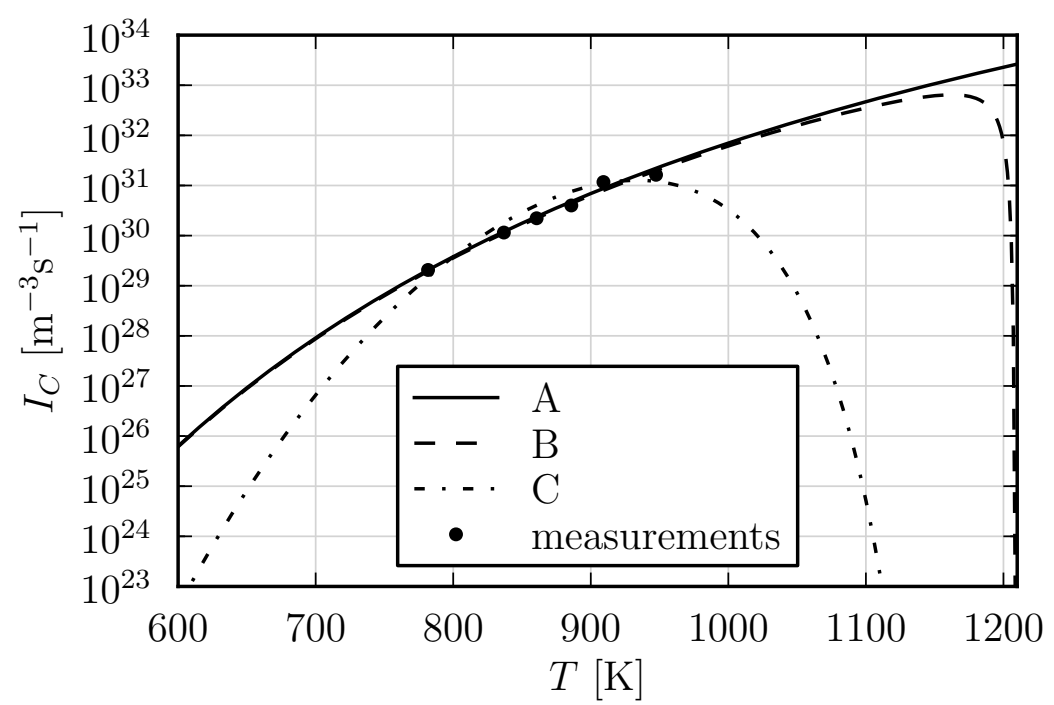

Figure 10.2.: Crystal nucleation rate for parameter sets A, B and C (Table 10.1). -: Measurements due to Marine \& Marfaing (1991).

Grigoropoulos et al., 2006; Marine \& Marfaing, 1991) as the "melting temperature of amorphous Germanium" (see section 10.1.5).

Figure 10.2 shows a comparison of the crystal nucleation rates resulting from the respective parameters as defined in this section.

\subsubsection{Characteristic crystallization time}

The characteristic crystallization time $t_{C}(T)$ as defined in $4.8 \mathrm{~b}$ can be obtained from $G_{C}(T)$ and $I_{C}(T)$. For completeness, $t_{C}(T)$ is shown in fig. 10.3. $t_{C}(T)$ blends $G_{C}(T)$ and $I_{C}(T)$ together, but because of the higher exponent of $G_{C}(T)$ in $4.8 \mathrm{~b}$, $G_{C}(T)$ is weighted more heavily than $I_{C}(T)$. It is difficult to spot this in fig. 10.3 . because the difference in peak temperature between $G_{C}(T)$ and $I_{C}(T)$, where this would be most readily apparent, is small for parameter set B, not applicable for parameter set $\mathrm{A}$, and zero for parameter set C.

\subsection{Substrate materials}

Typically, the substrate material used in explosive crystallization is glass Götzberger, 1955; Koba \& Wickersham, 1982; Shklovskij \& Kuz'menko, 1989; Vega et al., 2005) or 


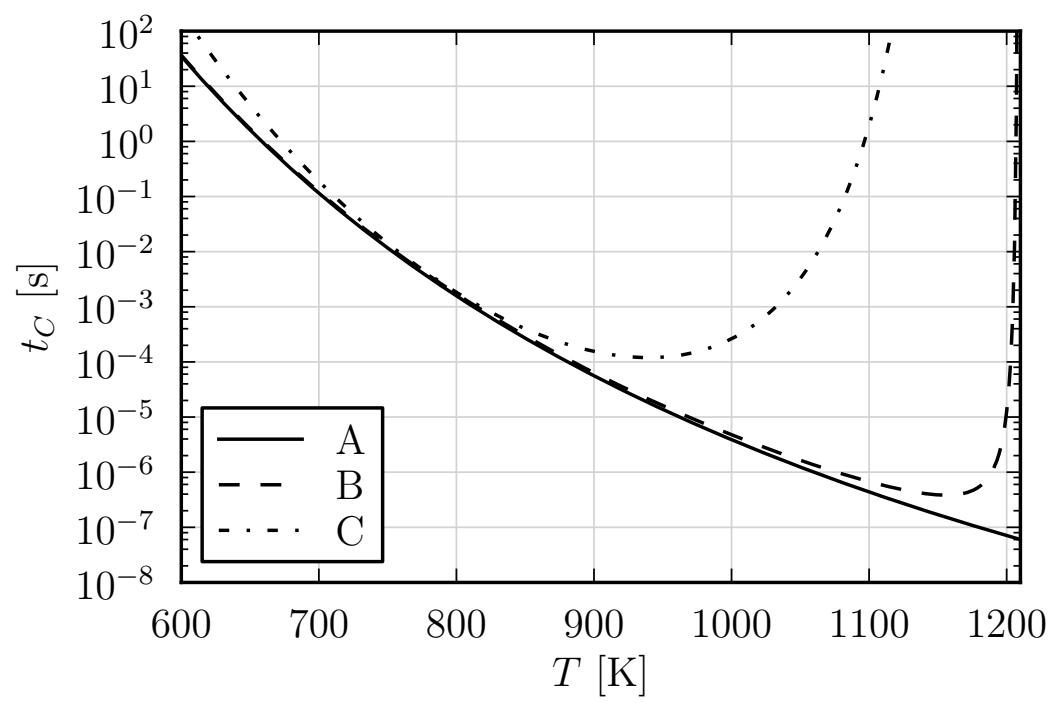

Figure 10.3.: Characteristic time of crystallization for parameter sets A, B and C (Table 10.1.

quartz Geiler et al., 1986; Geiler \& Heinig, 1985; Grigoropoulos et al., 2006; Ohdaira et al., 2009; Rogers et al., 2006; Spinella et al., 1998).

For quartz, a value of $\rho_{S} c_{p S}=2.31 \times 10^{6} \mathrm{~J} / \mathrm{m}^{3} \mathrm{~K}$, from Grigoropoulos et al. (2006), and $\alpha_{S}=1.2 \times 10^{-6} \mathrm{~m}^{2} / \mathrm{s}$, from Heinig \& Geiler (1985), are used.

\subsection{Parameters in experiments}

To demonstrate the main features of the mathematical model presented so far, appropriate values are chosen on the basis of data available in the literature.

\subsubsection{Film thickness}

A wide variety of film thickness values is given in the literature, ranging from $\delta_{L}=20-50 \mathrm{~nm}$ for free-standing films (Marine \& Marfaing, 1991), over films with a spatially varying thickness (Koba \& Wickersham, 1982, 1983), to $\delta_{L}=>10 \mu \mathrm{m}$. A value of $\delta_{L}=1.8 \mu \mathrm{m}$, as in Grigoropoulos et al. (2006); Rogers et al. (2006), is chosen for the main comparison of the presented model with experiments. 


\subsubsection{Substrate temperature}

The substrate temperatures reported in the literature for explosive crystallization of germanium vary over a range from room temperature (Shklovskij \& Kuz'menko, 1989; Takamori et al. 1972) to 400-560 K (Koba \& Wickersham, 1982) or 615-800 K (Grigoropoulos et al. 2006). Grigoropoulos et al. (2006) observe that a direct amorphousto-crystalline explosive crystallization process occurs only below a certain critical value of $T_{S}$, depending mainly on the film thickness $\delta_{L}$. For the presently chosen film thickness, values of $T_{S}=650$ and $700 \mathrm{~K}$ have been selected as representative. Additionally, variations of $T_{S}$ are investigated.

\subsubsection{Thermal contact resistance}

Values for $R_{i}$ from equation (5.16) are typically not reported in the available literature on explosive crystallization experiments. Thus, an estimation for reasonable values has to be inferred from other sources: Values were found in Zhao et al. (2004) for sputtered aluminum nitride films on silicon substrates $\left(R_{i}=7-8 \times 10^{-8} \mathrm{~m}^{2} \mathrm{~K} / \mathrm{W}\right)$ and in Rohde (1994) for sputtered titanium nitride on silicon $\left(R_{i}=2.5-10 \times 10^{-8}\right.$ $\left.\mathrm{m}^{2} \mathrm{~K} / \mathrm{W}\right)$ and for electroplated nickel on variously roughened steel substrates $\left(R_{i}=\right.$ $\left.2.6-13 \times 10^{-7} \mathrm{~m}^{2} \mathrm{~K} / \mathrm{W}\right)$. Thus, assuming a value of $R_{i} \approx 1 \times 10^{-8}-1 \times 10^{-6} \mathrm{~m}^{2} \mathrm{~K} / \mathrm{W}$ seems reasonable, lacking more relevant data.

Based on expected velocities $U \approx 1-10 \mathrm{~m} / \mathrm{s}$ and the above values for the various material and experimental parameters, equation (5.18) yields a possible range of $R \approx$ 0.01-10 for the dimensionless thermal contact resistance, and the scaled dimensionless thermal contact resistance $R^{*}$, cf. [5.32), can then be expected to have values of $R^{*} \approx 0.02-2$.

\subsubsection{Wave propagation velocity}

The wave propagation velocity $U$ is not a parameter of an experimental setup, but a result of the explosive crystallization process. Nevertheless, it is valuable to know the range of possible values of $U$. Unfortunately, the values of $U$ achieved in experiments are often not reported in the literature. Shklovskij \& Kuz'menko (1989) report values of $1-1.2 \mathrm{~m} / \mathrm{s}$, while Vega et al. (2005) observe $16 \pm 1 \mathrm{~m} / \mathrm{s}$ in an explosive crystallization process propagating in the direction normal to the film surface. Grigoropoulos et al. (2006) and Rogers et al. (2006) observe a range of velocities up to $8.75 \pm 0.15 \mathrm{~m} / \mathrm{s}$ when varying the substrate temperature $T_{S}$. 


\section{Results}

In this chapter, results are presented which show the most important features of the present explosive crystallization problem. Also, results are compared to experimental data for explosive crystallization in germanium films.

For germanium, only data for the crystal nucleation rate $I_{C}$ could be obtained. No data for the nucleation probability $n_{C}$, which is necessary for considering heterogeneous crystallization, could be found. Therefore, only homogeneous crystallization with the full set of rate equations, i.e. (8.11), is considered.

The superscript ^ denoting functions using the stretched space coordinate $z$ is omitted for simplicity.

\subsection{Illustrative solutions}

Figure 11.1 shows the main features of the explosive crystallization process, using an illustrative solution for an adiabatic case (i.e. $H=0$ ). Dimensionless temperature $\Theta$ and degree of crystallization $\xi$ are plotted vs. the stretched space coordinate $z$. To give an idea of the similarity coordinate $\eta$ associated with the $z$-coordinate, values of $\eta$ are also given in an upper, non-linear scale.

In fig. 11.1, the crystallization zone is quite small, compared to the pre-heating zone ahead of the crystallization zone.

At the bottom of fig. 11.1 is a plot of the cell size distribution in the computation domain. Note the (manually prescribed) cell size refinement in and near the crystallization zone, as well as near $z=1$.

Figure 11.2 shows an illustrative solution for a case including heat loss. Note that the length of the cooling zone behind the crystallization region is much larger than the length of the pre-heating region. This is illustrated by (4.19) and (7.9): The length of the pre-heating zone is $O(1)$, and the length of the cooling zone is $O\left([H \lambda]^{-2}\right)$. The dash-dotted line shows the asymptotic relation for $\Theta$ as $z \rightarrow 1$, equation 8.2 . 

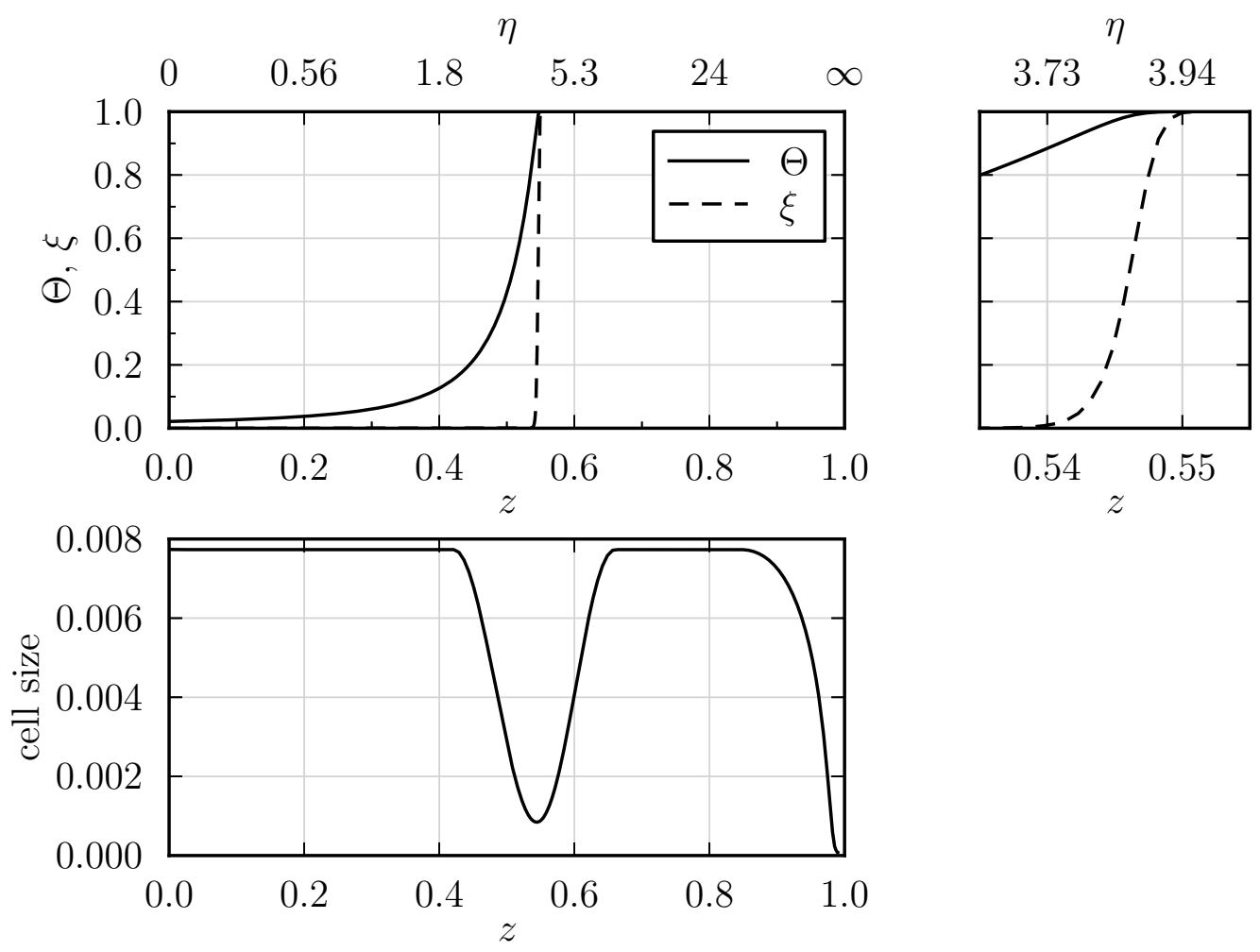

Figure 11.1.: Illustrative solution for a case without heat loss. Top left: Distribution of $\Theta$ and $\xi$ in the adiabatic case (i.e. $H=0$ ), using parameter set A (Table 10.1). Top right: Zoomed view of the crystallization zone. Bottom: Cell size distribution.

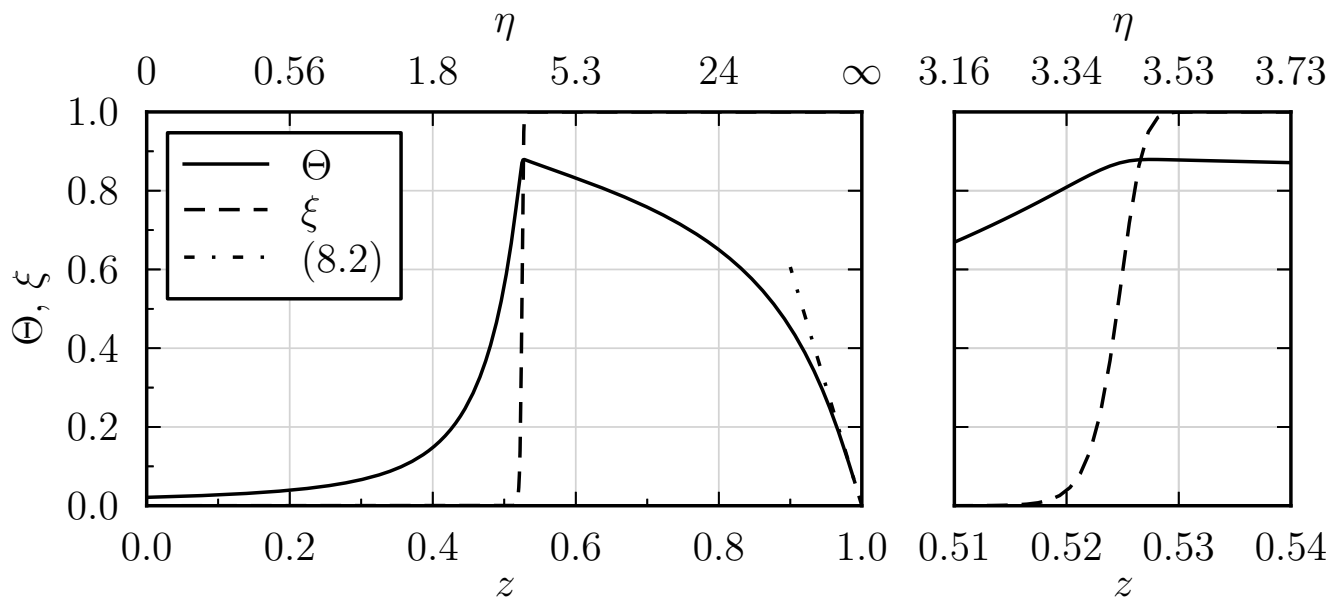

Figure 11.2.: Illustrative solution for a case with heat loss. Left: Distribution of $\Theta$ and $\xi$. Right: Zoomed view of the crystallization zone. $H=1.9 \times 10^{-2}$, other parameters using parameter set A (Table 10.1). Dash-dotted line: Asymptotic relation for $\Theta(z \rightarrow 1)$. 


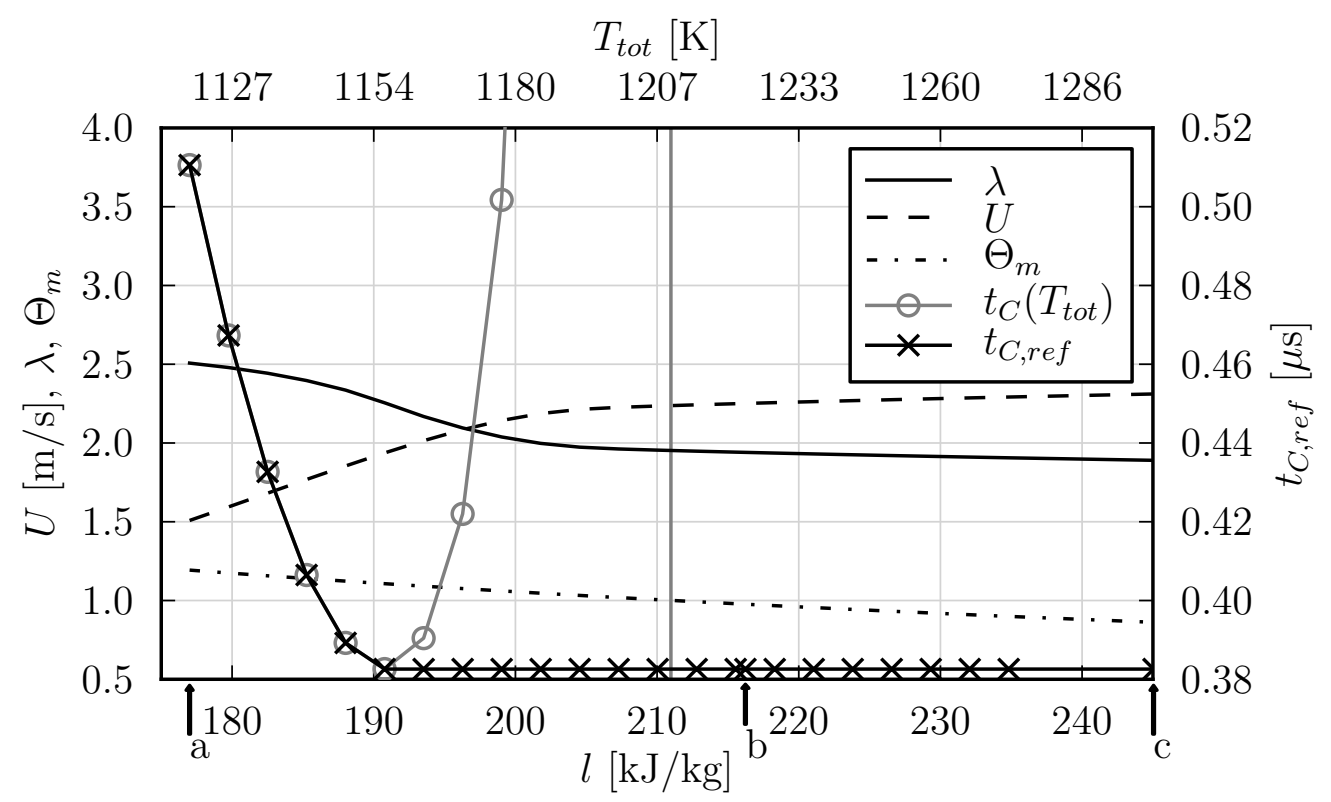

Figure 11.3.: Variation of $l$ for an adiabatic case, other parameters from parameter set B (Table 10.1). Symbols: Computed points. Vertical line: $T_{t o t}=T_{m}$. a-c refer to plots shown in fig. 11.4. $t_{C}\left(T_{t o t}\right)$ using $4.8 \mathrm{~b}$.

The agreement with the solution justifies using (8.2) to prescribe $\Theta$ at some $z<1$ as a boundary condition, cf. section 9.4 .

\subsection{Variation of the specific latent heat of melting}

The specific latent heat of melting $l$ is varied to examine the influence of a varying end temperature, while keeping $T_{S}$ constant, on the crystallization process. Both adiabatic cases and cases including heat loss are considered.

\subsubsection{Adiabatic case}

Figure 11.3 shows a plot of various relevant quantities when varying $l$, for an adiabatic case using the parameter set B. $t_{C}\left(T_{t o t}\right)$ tends to infinity as the total temperature $T_{\text {tot }}$ approaches $T_{m}$, but $t_{C, \text { ref }}$ does not. Consequently, $\lambda$ remains of the same order of magnitude, according to eq. (4.28). This confirms that the appropriate choice of $T_{\text {ref }}$ is using 4.9.

Figure 11.4 shows the wave shapes for two values of $l$ taken from fig. 11.3 . While the top, subcritical, case exhibits full crystallization and a sharp crystallization zone, 

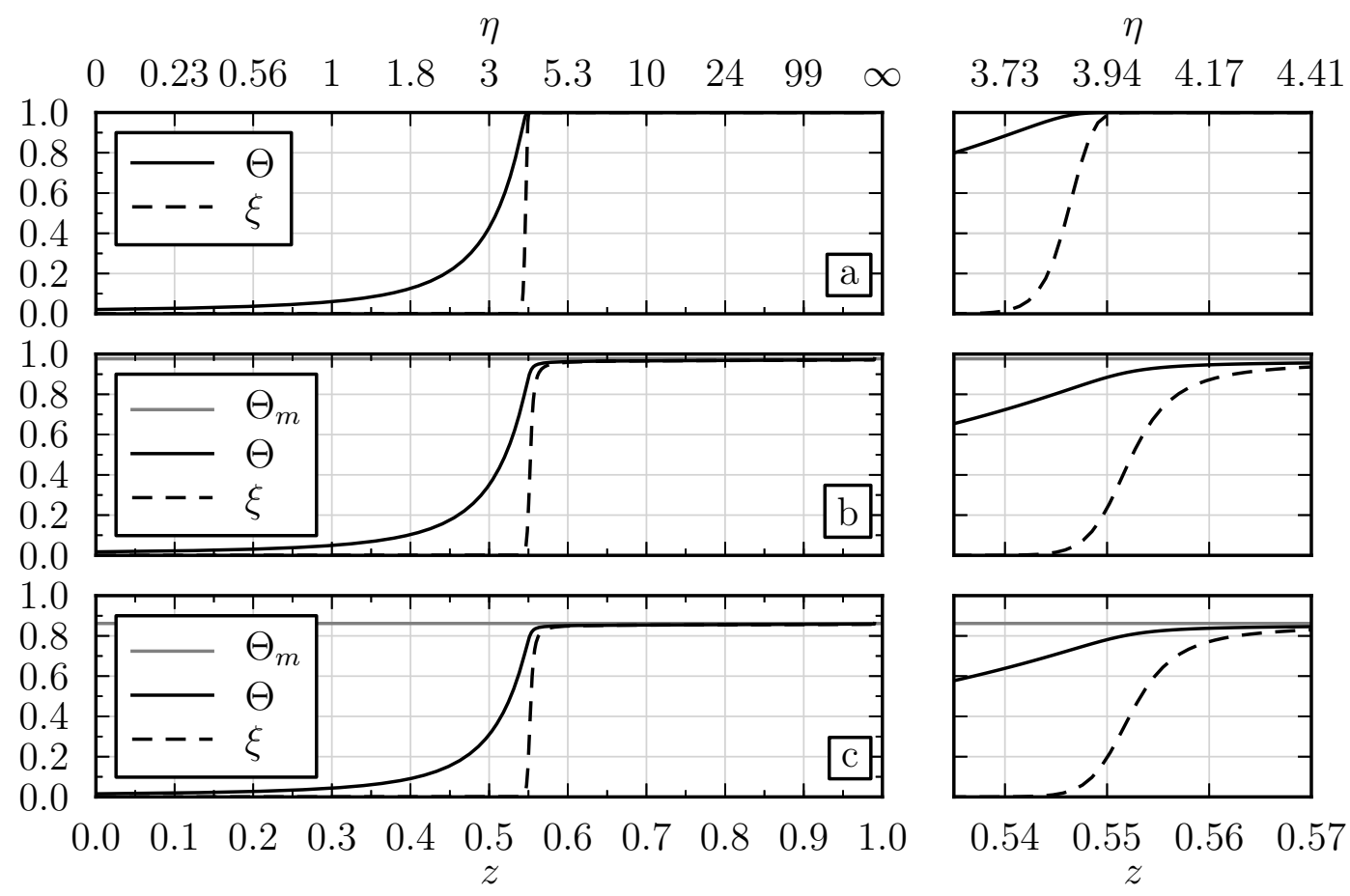

Figure 11.4.: Wave shapes for adiabatic cases, parameters as in fig. 11.3. Left: Whole z-range. Right: Zoomed view of the crystallization zone. $a: l=177$ $\mathrm{kJ} / \mathrm{kg}, b: l=216.2 \mathrm{~kJ} / \mathrm{kg}, c: l=245.0 \mathrm{~kJ} / \mathrm{kg}$. For $b, \Theta_{m}=0.98$, so the gray line is near the top of the plot.

in the middle and bottom figures, showing the highly supercritical cases $\left(T_{a d}=T_{m}\right)$, the incomplete crystallization and a wider crystallization zone can be recognized. Furthermore, b and c are cases where $T_{t o t}>T_{a d}$ (i.e. $\Theta_{m}<1$ ).

\subsubsection{Case including heat loss}

Figure 11.5 shows the same quantities as fig. 11.3 , but with heat loss to the substrate taken into account. Additionally, fig. 11.5 shows a plot of the maximum temperature $\Theta_{\max }$, which is defined as

$$
\Theta_{\max }=\max [\Theta(z)]
$$

In the adiabatic case, $\Theta_{\max }=\min \left(1, \Theta_{m}\right)$. The basic shapes of the plots remain similar to the adiabatic case. Note that the wave propagation velocity $U$ is always lower for the case with heat loss, as expected from results in Buchner \& Schneider (2010b). 
$T_{\text {tot }}[\mathrm{K}]$

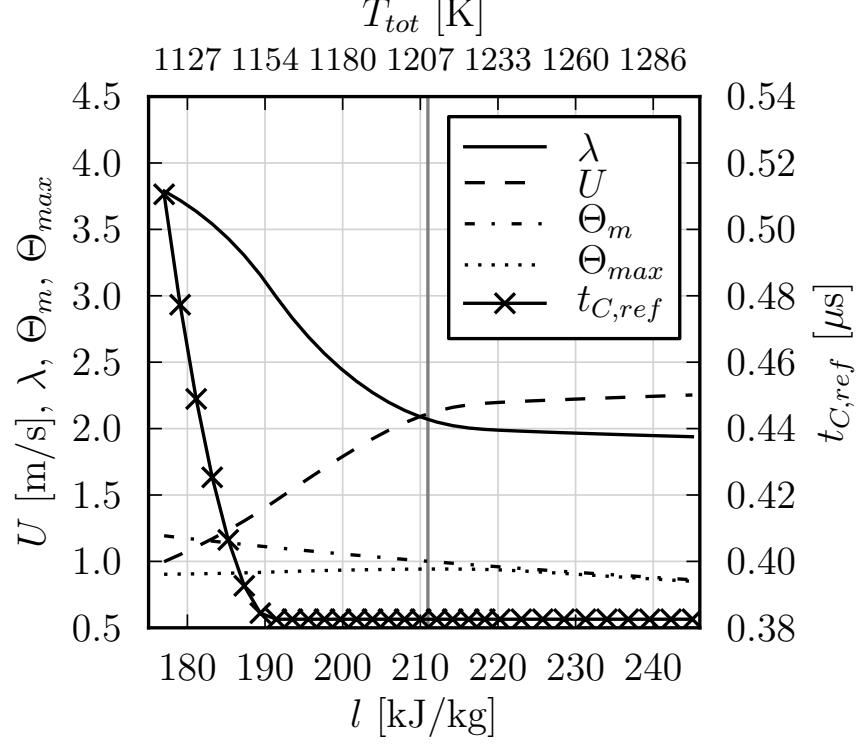

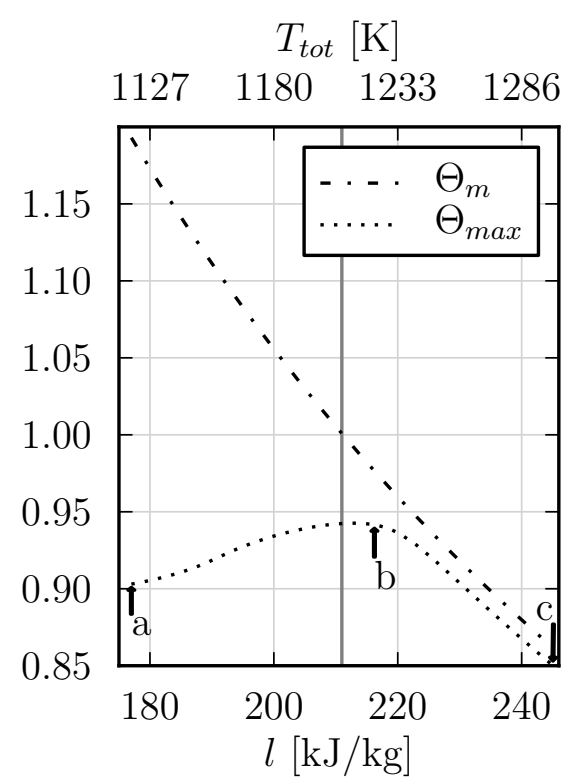

Figure 11.5.: Variation of $l$ for a case including heat loss. $H=0.019$, other parameters from parameter set B (Table 10.1). Notation as in fig. 11.3. Left: Relevant quantities, analogous to fig. 11.3. Right: Detailed view of $\Theta_{m}$, $\Theta_{\max }$. a-c refer to plots shown in fig. 11.6, with $l$ values identical to fig. 11.3. Vertical line: $T_{\text {tot }}=T_{m}$.

The plot of $\Theta_{\max }$ on the right side of fig. 11.5 shows an interesting behaviour. To visualize the differences in $\Theta$ and $\xi$, respectively, when varying $l$, plots for the values of $l$ indicated by letters on the right side of fig. 11.5 are shown in fig. 11.6. The value of $\Theta_{m}$ is indicated by the gray line.

Figure 11.6 shows a subcritical situation at the low end of the $l$ range. From there, $\Theta_{\text {max }}$ is slowly growing with $l$ until $\Theta_{m} \approx 1$. This is due to the fact that in this region $\Theta_{\text {max }}$ is only slightly above the temperature of fastest crystallization (i.e. minimum $t_{C}$ ), and most of the latent heat get released very quickly, diminishing the influence of the heat loss on $\Theta_{\max }$ (fig. 11.6p). With further rising $l, \Theta_{\max }$ approaches $\Theta_{m}$, so the crystallization at $\Theta_{\max }$ slows down and the influence of the heat loss is more pronounced. The slowed crystallization is also responsible for a wider crystallization region, as in the adiabatic case.

\subsubsection{Plateau region of nearly constant temperature}

With heat losses taken into account, $\Theta_{\max }$ never reaches $\Theta_{m}$, as opposed to the adiabatic case. This is, on the one hand, because the heat loss into the substrate 


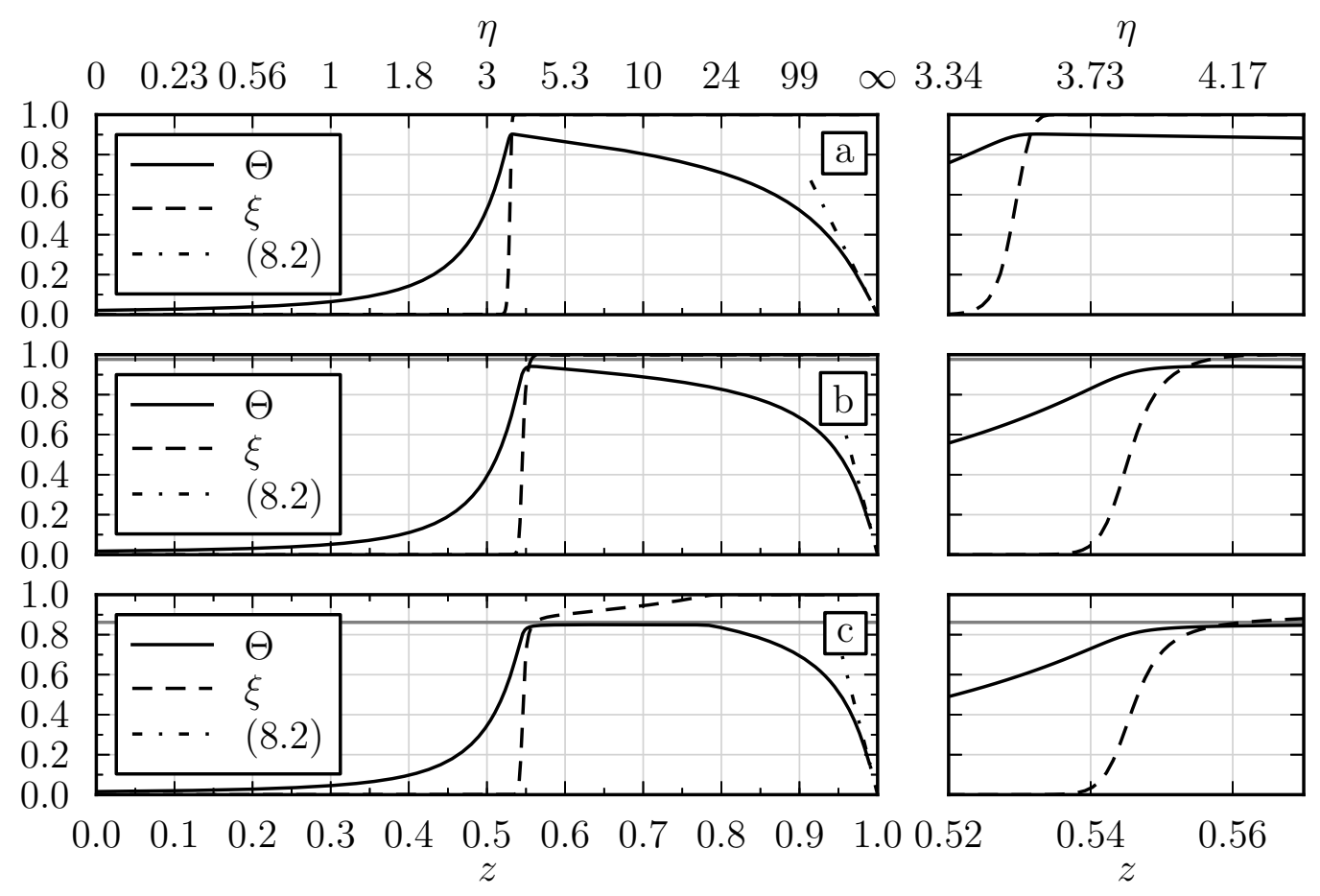

Figure 11.6.: Wave shapes for a-c in fig. 11.5. Top: $l=177.0 \mathrm{~kJ} / \mathrm{kg}$, middle: $l=216.2$ $\mathrm{kJ} / \mathrm{kg}$, bottom: $l=245.0 \mathrm{~kJ} / \mathrm{kg}$. Left: Whole z-range, right: Zoomed view of the crystallization zone. Gray horizontal lines: $\Theta_{m}$.

reduces $\Theta$. On the other hand, consider the supercritical region of $t_{C}$, i.e. where $\mathrm{d} t_{C} / \mathrm{d} \Theta>0$ (cf. fig. 10.3 , curve B). There, crystallization, and thus release of latent heat, slows down with increasing temperature. $\Theta_{m}$ can never be reached, because at that point, there's no release of latent heat due to terminated crystallization $\left(t_{C} \rightarrow \infty\right)$, but heat loss into the substrate is still in effect.

This leads to an interesting effect if $l$ is increased even more, compared to fig. $11.6 \mathrm{~b}$, as can be seen in fig. 11.6. At $z \approx 0.6$, a highly supercritical temperature range with its very slow crystallization is reached with crystallization still incomplete, i.e. $\xi<1$. A temperature drop due to heat loss would lead to accelerated crystallization and enthalpy release, which would raise the temperature. This leads to the wave attaining a temperature where the heat release due to crystallization and the heat loss into the substrate balance each other. This is in contrast to the subcritical region of $\Theta$, where $\mathrm{d} t_{C} / \mathrm{d} \Theta<0$ (cf. fig. 10.3 , curve B).

In fig. 11.6 c, for $0.6 \lesssim z \lesssim 0.75$, the temperature stays at a nearly constant level slightly below $\Theta_{m}$ until all the residual amorphous material has crystallized and the balance cannot be maintained anymore, whereupon cooling down to $\Theta=0$ sets in. 


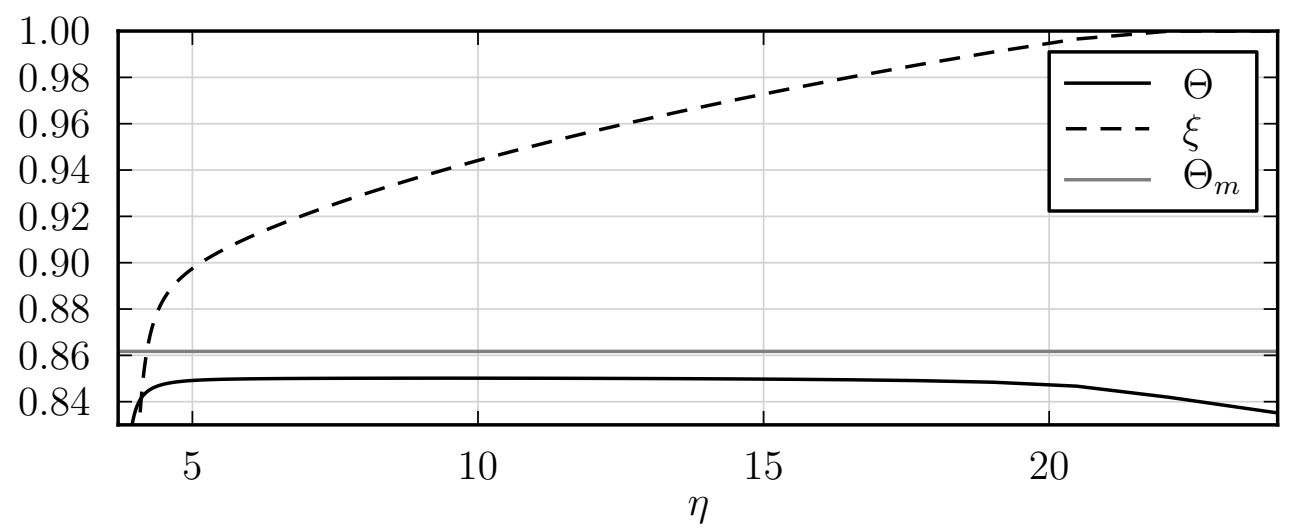

Figure 11.7.: Detail view of fig. 11.6 , using the unstretched space coordinate $\eta$.

This can also be seen in fig. 11.7, which shows a detailed view of fig. 11.6c, using the unstretched space coordinate $\eta$.

$\Theta$ being nearly constant in this plateau region means that nearly no heat conduction in longitudinal direction takes place. This is in contrast to the adiabatic case, where the enthalpy released in the wide post-crystallization zone has to be conducted solely towards $\eta \rightarrow-\infty$, requiring a longitudinal temperature gradient.

\subsubsection{Non-local influence on heat loss}

Another remarkable detail of the plateau region is shown in fig. 11.7; Due to the constant $\Theta$, heat loss into the substrate can only be compensated by the release of latent heat due to crystallization. It is often assumed in the literature - e.g. in Grigoropoulos et al. (2006); Heinig \& Geiler (1985); Kurtze (1986); Shklovskij \& Ostroushko (1996) - that the heat loss into the substrate can be described by a local heat transfer coefficient (see also section 5.2.2). If that were the case, a constant temperature would require a constant rate of crystallization, i.e. $\mathrm{d} \xi / \mathrm{d} \eta=$ const. In fig. 11.7 it can be seen that $\mathrm{d} \xi / \mathrm{d} \eta$ is in fact not constant in the plateau region, therefore corroborating our finding of a non-local influence of the temperature distribution on the heat loss, as described by (5.14)!

\subsection{Variation of heat loss parameter $\mathrm{H}$}

The coefficient $H \lambda$ in (9.1) provided a hint at looking at the dependence of the eigenvalue $\lambda$ on $H \lambda$ (Buchner \& Schneider, 2010b). This is shown in fig. 11.8, It contains 


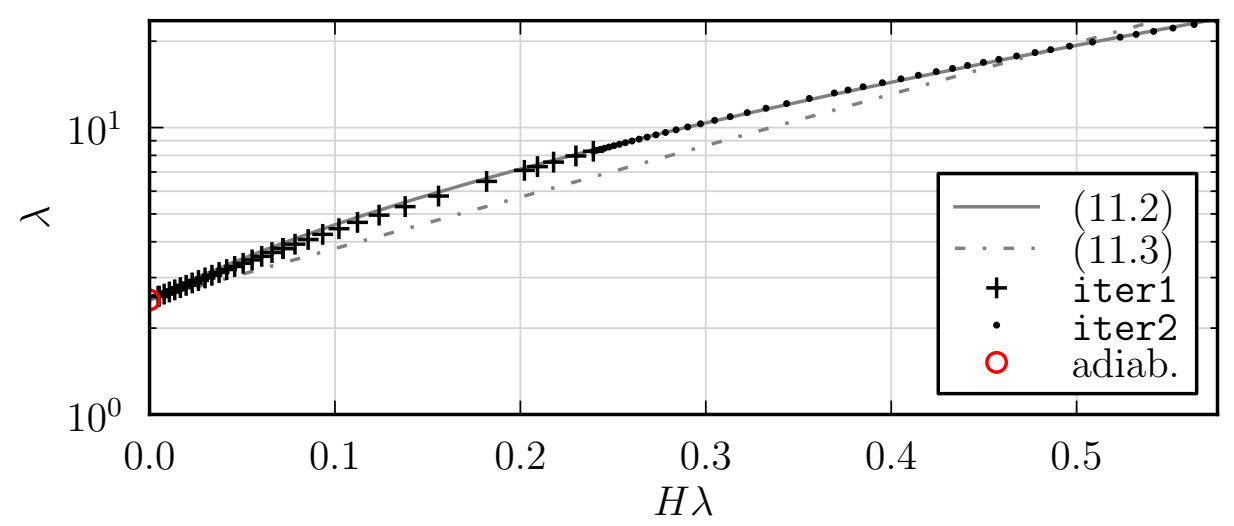

Figure 11.8.: $\lambda$ vs. $H \lambda$, other parameters from parameter set $\mathrm{B}$ (Table 10.1). Solid line: 11.2 , with $\lambda_{a d}=2.51, P_{1}=8.53$ and $P_{2}=2.18$. Dash-dotted line: 11.3, with $\lambda_{a d}=2.51, P_{1}=4.13$.

results obtained from a computation without heat loss as well as from computations using the two different iteration schemes iter1 and iter2 (see section 9.3.2). To preserve clarity, data points have been omitted where they are too densely spaced.

Just as in Buchner \& Schneider (2010b), the results obtained here can be approximated with a function of the form

$$
\lambda=\lambda_{a d}+P_{1}\left[\exp \left(P_{2} H \lambda\right)-1\right]
$$

with $\lambda_{a d}$ being the value of $\lambda$ in the adiabatic limit, i.e. for $H=0$, and $P_{1}, P_{2}$ being constant parameters to be determined with the method of least squares. An analytic proof of this relationship is not yet available. However, an exponential relationship has been obtained for heterogeneous crystallization with an asymptotic expansion for large activation energies (Schneider, 2010). As can be seen in fig. 11.8, a purely exponential function of the form

$$
\lambda=\lambda_{a d} \exp \left(P_{1} H \lambda\right)
$$

is not sufficient to describe the numerical results.

\subsubsection{Critical value of $H$}

The results in fig. 11.8 and eq. (11.2) are implicit with regard to the eigenvalue $\lambda$. The explicit relationship between $\lambda$ and $H$, using the same data, is shown in 


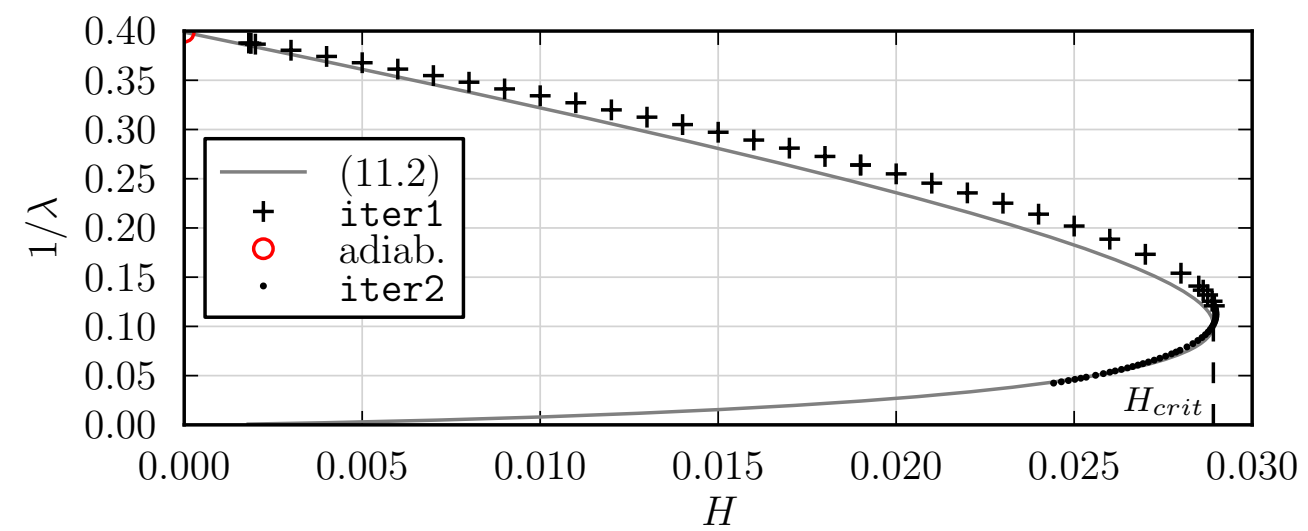

Figure 11.9.: $1 / \lambda$ vs. $H$, other parameters from parameter set B (Table 10.1). $1 / \lambda \propto$ $U$. $H$ characterizes heat loss effect. Solid line: 11.2, parameters as in fig. 11.8, Dashed line: $H_{\text {crit }}=2.89 \times 10^{-2}$, i.e. the maximum attainable value for $H$.

fig. 11.9. As in Buchner \& Schneider (2010b), $1 / \lambda$ is plotted instead of $\lambda$, because $1 / \lambda$ is proportional to the velocity of the crystallization wave $U$, see (4.28).

Remarkably, for values of $H$ smaller than a certain maximum value, $H_{\text {crit }}$, there exist two solutions, i.e. an upper and a lower solution branch. For $H=H_{\text {crit }}$ there is one solution, while for $H>H_{\text {crit }}$ no steady-state solution exists.

To be able to successfully obtain results near $H_{c r i t}$, and on the lower branch, it was necessary to switch to the alternative iteration scheme iter2. This scheme resulted in a smaller maximum possible step size when varying computation parameters (see section 9.3.2), which is evident in the dense distribution of points near $H_{\text {crit }}$, obtained using iter2. Additionally, on the lower branch, it was necessary to contract the computation domain, i.e. slightly reduce $z_{r}$ (cf. section 9.4), to ensure convergence. This reduction, e.g. from $z_{r}=0.99$ to $z_{r}=0.98$, was confirmed not to have an significant impact on the computation results.

According to (4.27), $H$ is inversely proportional to the layer thickness $\delta_{L}$, while all other quantities occuring in 4.27) are material properties or, in the case of $t_{C, \text { ref }}$, crystallization quantities depending on material properties and the reference temperature. Therefore, for given materials and a constant reference temperature, the maximum value $H_{\text {crit }}$ can be interpreted as a minimum layer thickness $\delta_{L, \text { crit }}$, below which explosive crystallization is not possible. This result is in accord with observations described in the literature (Götzberger, 1955; Koba \& Wickersham, 1982; Shklovskij \& Kuz'menko, 1989; Takamori et al., 1972). 
If, on the other hand, the material properties of the layer, including the crystallization parameters and the layer thickness, are fixed, $H_{\text {crit }}$ provides an upper limit for $k_{S} \rho_{S} c_{p S}$ that must not be exceeded in order to have a solution. This essentially limits the thermal conductivity of the material of the substrate Buchner \& Schneider, $2010 b)$.

\subsubsection{Plateau solutions}

Increasing the value of $l$ leads to the appearance of a plateau region in $\Theta$, cf. fig. 11.6, plot $c$. Varying $H$ with this new $l$ is shown in fig. 11.10. The plateau markedly

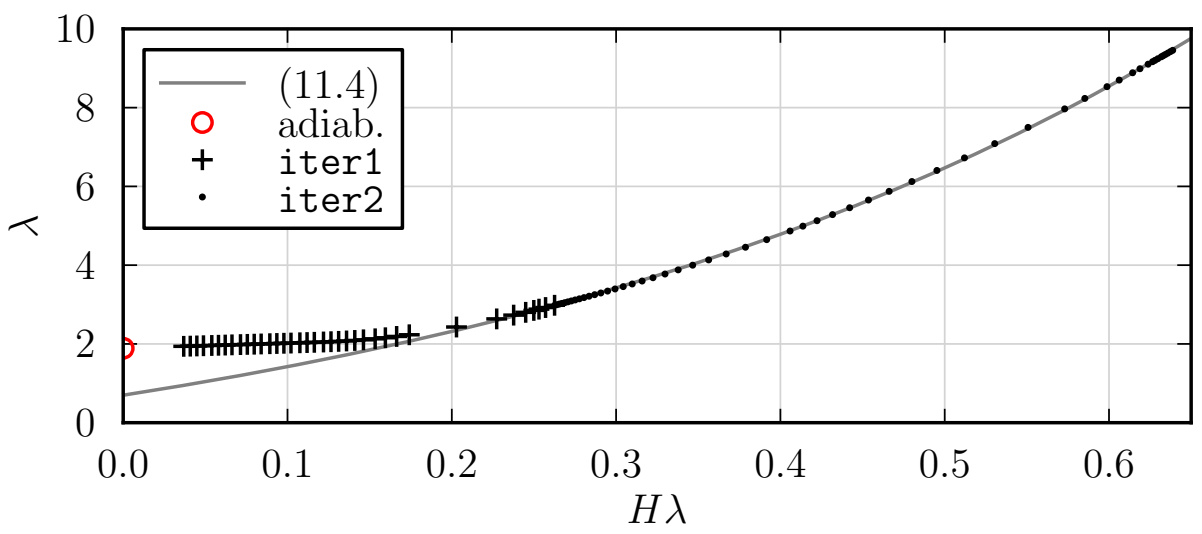

Figure 11.10.: $\lambda$ vs. $H \lambda$, with $l=245.0 \mathrm{~kJ} / \mathrm{kg}$, other parameters from parameter set B (Table 10.1). Solid line: 11.4). Using only results where $H \lambda>0.2$, $S_{1}=0.70, S_{2}=3.09$ and $S_{3}=2.11$. Data points omitted where too densely spaced to preserve clarity.

changes the characteristic of the plot: In the region where a plateau occurs, i.e. for $H \lambda \lesssim 0.2$, the relationship of $\lambda$ to $H \lambda$ is approximately linear instead of exponential. As the influence of the heat loss increases, the plateau grows shorter in extent, until it finally disappears and the previously known exponential relationship is re-established.

This is illustrated by employing a slightly modified eq. (11.2) to approximate the computation results:

$$
\lambda=S_{1}+S_{2}\left[\exp \left(S_{3} H \lambda\right)-1\right]
$$

Only results which don't exhibit a plateau region, i.e. $H \lambda>0.2$, are used to determine the parameters $S_{1}, S_{2}$ and $S_{3}$ in (11.4). This shows that the exponential relationship stays valid outside of the region where $\Theta$ plateaus occur.

Figure 11.11 shows the same data using the explicit $1 / \lambda-H$ relationship. The 


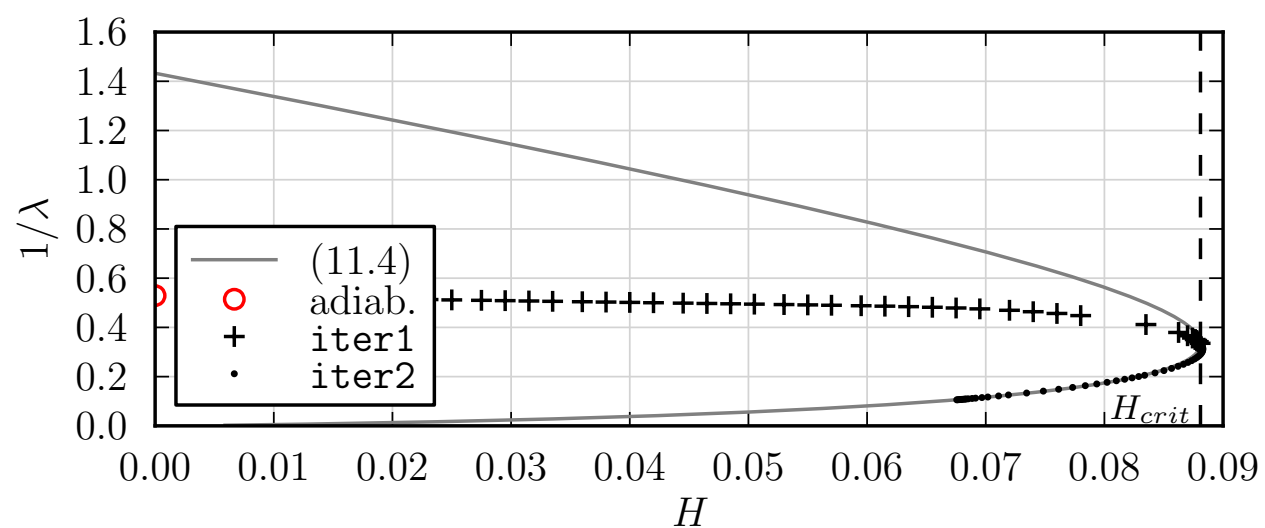

Figure 11.11.: $1 / \lambda$ vs. $H$. Solid line: 11.4), parameters as in fig. 11.10, $H_{\text {crit }}=$ $8.83 \times 10^{-2}$.

difference between the results in the plateau region and $(11.4)$ is even more clear here. In addition, figure 11.12 shows the variation of the maximum attained temperature $\Theta_{\max }$ in relation to the melting temperature $\Theta_{m}$ and the temperature of fastest crystallization $\Theta_{\text {ref }}$, for these computations. The plateau region occurs where $\Theta_{\max } \lesssim$ $\Theta_{m}$.

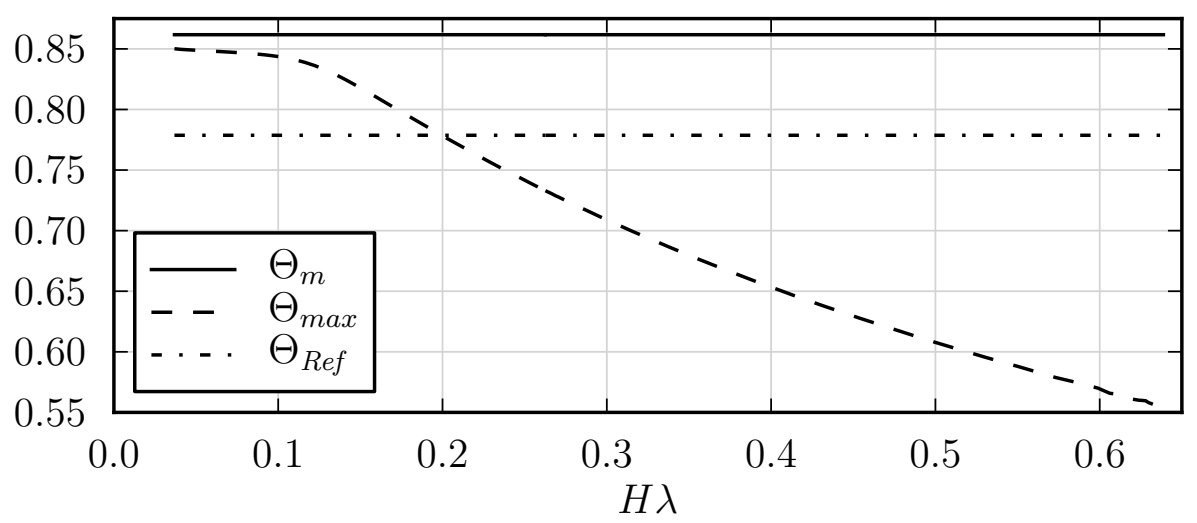

Figure 11.12.: $\Theta_{\max }$ in relation to $\Theta_{m}$ and $\Theta_{\text {ref }}$, vs. $H \lambda$, parameters as in fig. 11.10 . The plateau region is visible on the left side of the plot, where $\Theta_{\max } \lesssim$ $\Theta_{m}$. The transition between effectively super- and subcritical wave is also recognizable where $\Theta_{\max } \approx \Theta_{\text {ref }}$. 


\subsubsection{Effective criticality}

The classification of explosive crystallization waves so far relies on the relation of $T_{a d}$ to $T_{r e f}$. Waves are classified as subcritical, critical, and supercritical if $T_{a d}<T_{\text {ref }}$, $T_{a d} \approx T_{r e f}$, and $T_{a d}>T_{\text {ref }}$, respectively, cf. section 4.2 .

Alternatively, we can define an effective criticality, where instead of $T_{a d}$, which is the maximum attained temperature only in the case without heat loss, $T_{\max }$ is used for classification: Waves are classified as subcritical, critical, and supercritical if $T_{\max }<T_{\text {ref }}, T_{\max } \approx T_{\text {ref }}$, and $T_{\max }>T_{\text {ref }}$, respectively.

Figure 11.12 illustrates the impact of the improved classification scheme: According to the "simple" classification, the explosive crystallization waves are supercritical everywhere, i.e. $\Theta_{a d}=\Theta_{m}>\Theta_{\text {ref }}$. A classification according to the new effective criticality shows a different picture: An initially supercritical $\left(\Theta_{\max }>\Theta_{\text {ref }}\right)$ solution becomes critical $\left(\Theta_{\max } \approx \Theta_{\text {ref }}\right)$ with increasing heat loss, and even subcritical $\left(\Theta_{\max }<\Theta_{\text {ref }}\right)$ !

\subsection{Variation of thermal contact resistance}

In this section, the influence of the thermal contact resistance on $\lambda$ is examined, using the same parameters as for the comparison to experiments, cf. section 11.6. Figure 11.13 shows $1 / \lambda$ for varying $R$. As $R \rightarrow \infty, 1 / \lambda$ tends toward the value without considering heat loss. This is expected because a growing $R$ reduces the influence of the heat loss into the substrate. As $R \rightarrow 0,1 / \lambda$ tends toward the value for $R=0$, i.e. a computation without considering thermal contact resistance. Thus, fig. 11.13 validates the approximations for $R \ll 1$, cf. eq. (5.33), and $R \gg 1$, cf. eq. (5.38), by demonstrating that they show the correct behaviour when approaching the limiting cases of $R=0$ and $H=0$, respectively.

Interestingly, the results for the $R \ll 1$ approximation (solid blue line in fig. 11.13) are linear in $R$. This can also be seen in an alternative presentation of $\lambda$ vs. the scaled thermal contact resistance $R^{*}$, see figure 11.14. The behaviour is accurately described by

$$
\lambda=\lambda_{0}+R^{*} \lambda_{1}
$$

The reason for this linear dependence is not yet clear, and might warrant further investigation. 


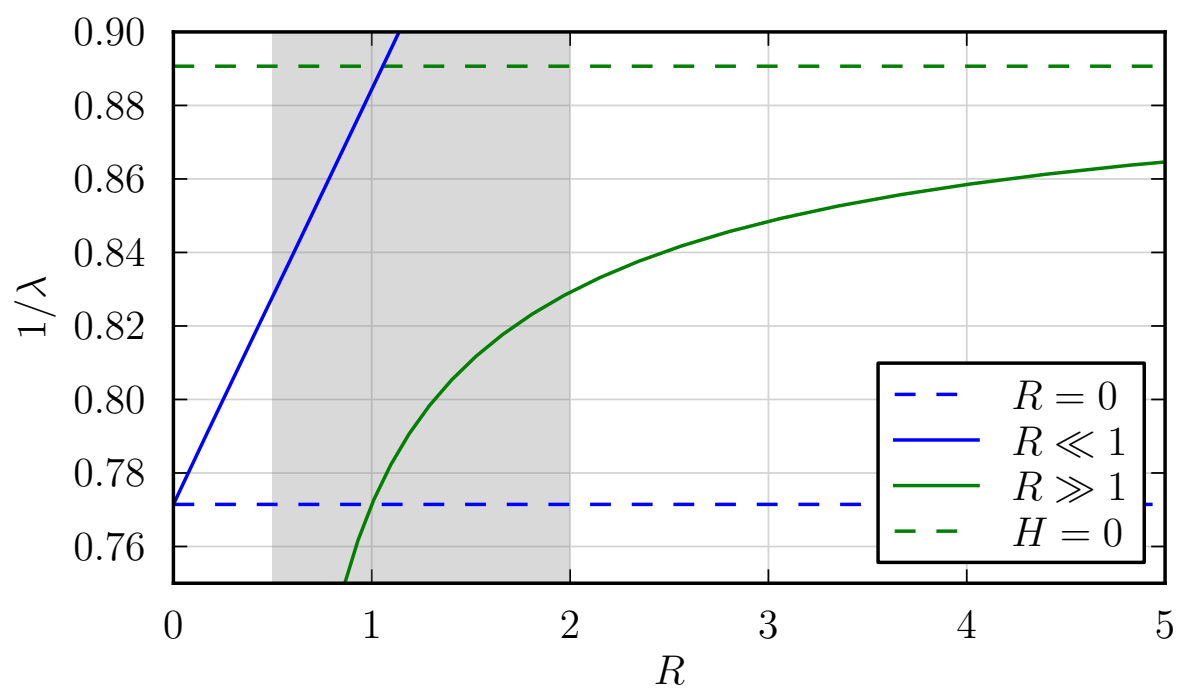

Figure 11.13.: $1 / \lambda$ for varying $R$, using parameter set $\mathrm{C}$ and a kinetic prefactor $P=1500$. Approximations $R \ll 1$ (5.33) and $R \gg 1$ (5.38), and their limiting cases $R=0$ and $H=0$, respectively. Shaded area: approximate overlap region $0.5<R<2$ where neither approximation is valid.

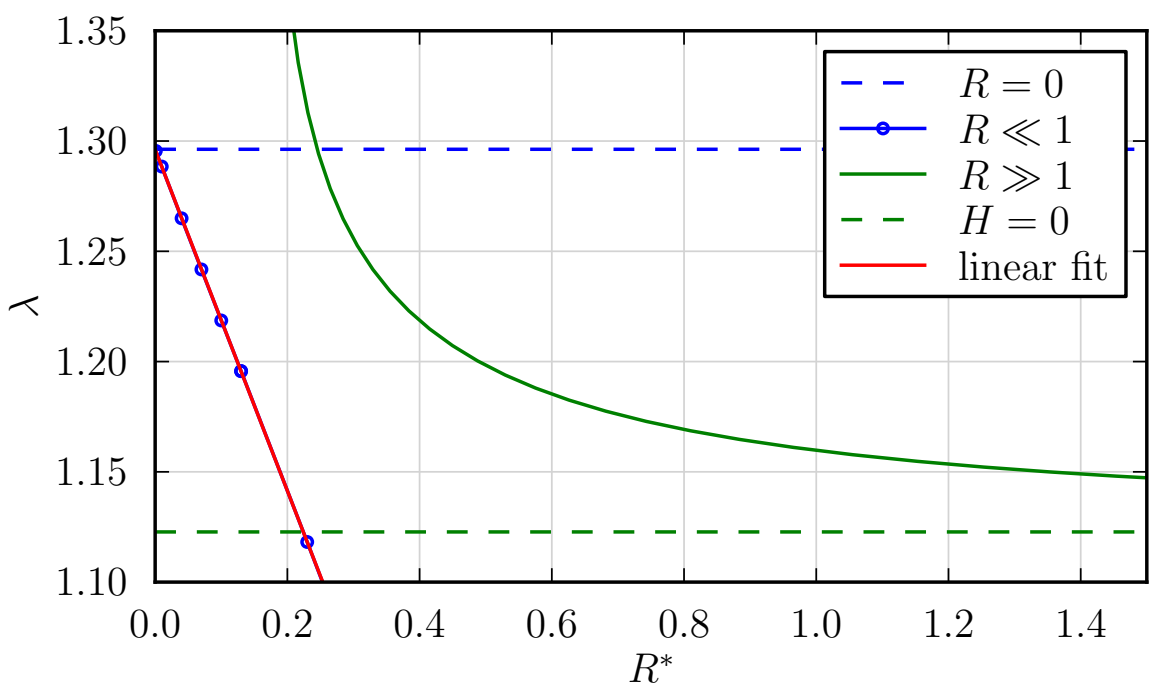

Figure 11.14.: Alternative presentation of figure 11.13, $\lambda$ vs. $R^{*}$, using parameter set $\mathrm{C}$ and a kinetic prefactor $P=1500$. Red line: linear fit to the visible portion of data for $R \ll 1$; coefficients of (11.5): $\lambda_{0}=1.296$, $\lambda_{1}=-0.774$. 


\subsection{Length of crystallization zone}

Figure 11.15 shows the length of the crystallization zone when varying $T_{S}$, using the same parameters as for the comparison to experiments, cf. section 11.6.

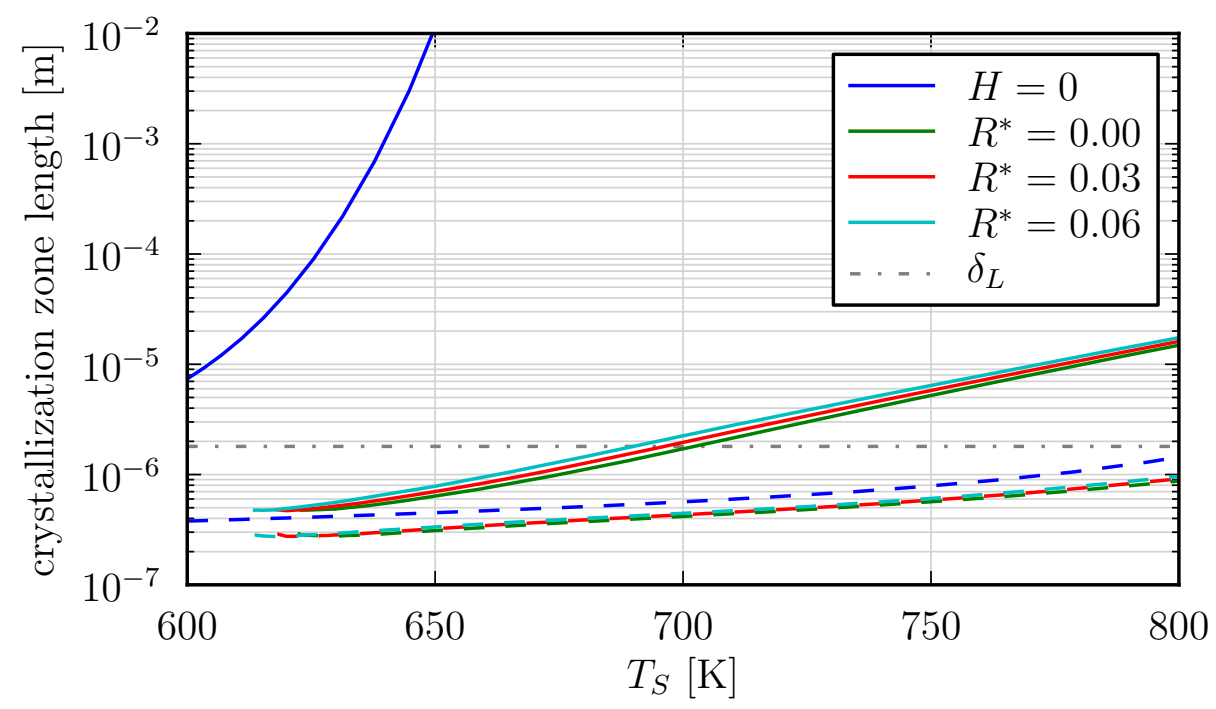

Figure 11.15.: Length of the crystallization zone vs. $T_{S}$, using parameter set $\mathrm{C}$ and a kinetic prefactor $P=1500$. Solid lines: length of zone $0.01 \leq \xi \leq 0.99$. Dashed lines: length of zone $0.01 \leq \xi \leq 0.5$. Dash-dotted line: Layer thickness $\delta_{L}$.

The crystallization zone is (arbitrarily) defined as the region where $0.01 \leq \xi \leq 0.99$ (solid lines). It can be seen that the crystallization zone for the computations without heat loss is much longer than for the computations with heat loss. This can be understood as follows: In the supercritical crystallization regime encountered in these computations, the crystallization rate decreases exponentially as $\Theta$ approaches $\Theta_{m}$ (cf. figure 10.3). In the case without heat loss, this means that as $T_{a d}$ approaches $T_{m}$, crystallization slows down as $\Theta \rightarrow 1$. This leads to an elongated crystallization zone, the length of which, in the limit of $T_{a d} \rightarrow T_{m}$, becomes infinite.

In the computations including heat loss, the heat loss into the substrate and the associated temperature decrease accelerates crystallization. The enthalpy release due to crystallization is balanced by the heat loss into the substrate. This leads to the appearance of plateau regions in $\Theta$, as detailed in section 11.2.3. The more $T_{a d}$ approaches $T_{m}$, the longer the plateau region becomes.

Figure 11.15 shows that the big differences between computations with and without heat loss primarily arise in the later part of the crystallization process: The 


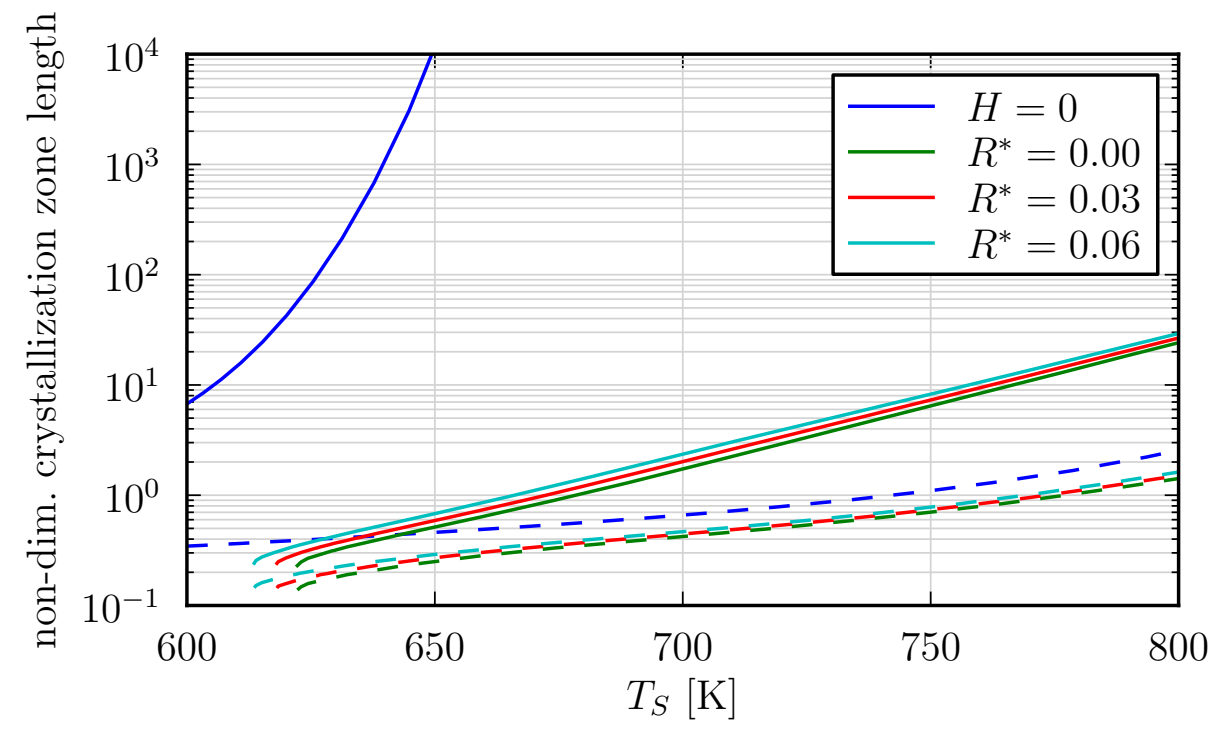

Figure 11.16.: Nondimensional length of the crystallization zone vs. $T_{S}$, using parameter set $\mathrm{C}$ and a kinetic prefactor $P=1500$. Same data as fig. 11.15, but with the length of the crystallization zone referred to $L_{r e f}$. Solid lines: length of zone $0.01 \leq \xi \leq 0.99$. Dashed lines: length of zone $0.01 \leq \xi \leq 0.5$.

dashed lines indicate the length of the region where the first half of crystallization happens, i.e. $0.01 \leq \xi \leq 0.5$. This length turns out to be very similar for all shown computations, and does not vary very much over the substrate temperature range examined.

Figure 11.15 also indicates $\delta_{L}$ with a dash-dotted line. It can be seen that the thickness of the crystallization zone varies from being significantly smaller than $\delta_{L}$ for small values of $T_{S}$ to being significantly larger than $\delta_{L}$ for large values of $T_{S}$. Also, it should be noted that $L_{r e f}$ is $O(1 \mu \mathrm{m})$, and therefore comparable to $\delta_{L}$.

Figure 11.16 shows the same computations as figure 11.15, but with the length of the crystallization zone given in nondimensional coordinates, i.e. multiples of $L_{r e f}$.

\subsection{Comparison with experiments}

To compare the present model to experimental data, results reported in Grigoropoulos et al. (2006) have been selected. That article reports on explosive crystallization results in germanium films on a quartz substrate, for which the necessary material and crystallization data could be obtained elsewhere, see table 10.1. The article 
includes data on the behaviour of the wave propagation velocity $U$ with varying substrate temperature $T_{S}$. Since $U$ is a central result of our model, those experimental data are very useful. Very often, values of $U$ are not reported in articles concerning explosive crystallization experiments.

A steady-state process is reported in Grigoropoulos et al. (2006) after the crystallization front propagated approximately $50 \mu \mathrm{m}$, in a square sample with a side length of $5 \mathrm{~mm}$. Therefore, the velocity results can be assumed to reflect a fully developed, steady state wave propagation, which the present model requires.

\subsubsection{Kinetic prefactor}

To achieve wave speeds $U$ comparable with the results in the Grigoropoulos et al. (2006), it was necessary to adjust the given values for the crystallization parameters (see parameter set $\mathrm{C}$ in table 10.1). Changing the kinetic prefactors, i.e. $G_{0}$ and $I_{0}$ in (3.18) and (3.21), respectively, will not change the shape of the non-dimensional kinetic curves $G(\Theta)$ and $I(\Theta)$, but will influence $t_{C, r e f}$ according to (4.11) and 4.8b). $t_{C, \text { ref }}$ will change $U$, via the definition of $\lambda$ given in (4.28), but also the value of $H$, cf. (4.27). Both $G_{0}$ and $I_{0}$ are multiplied with a (common) prefactor $P$. In (3.18) and (3.21), respectively, these quantities are replaced, such that

$$
\begin{gathered}
G_{0} \text { becomes } P G_{0}, \\
I_{0} \text { becomes } P I_{0} .
\end{gathered}
$$

It should be noted that a similar adjustment was also carried out in Grigoropoulos et al. (2006).

A value of $P=1500$ gives reasonable results around $T_{S}=700 \mathrm{~K}$, as can be seen in figure 11.17. It can be seen that introducing a small thermal contact resistance has an appreciable effect, shifting the $U\left(T_{S}\right)$ curve towards lower substrate temperatures. For these computations, the model employing the approximation for small thermal contact resistance has been used. The necessary condition $R \ll 1$ is fulfilled for the results shown in figure 11.17 .

It should be noted that the behaviour of $U\left(T_{S}\right)$ in fig. 11.17 is in qualitative agreement with theoretical results for an "Explosive Solid Phase Nucleation process" in silicon (Heinig \& Geiler, 1985, fig. 6, p. 428). 


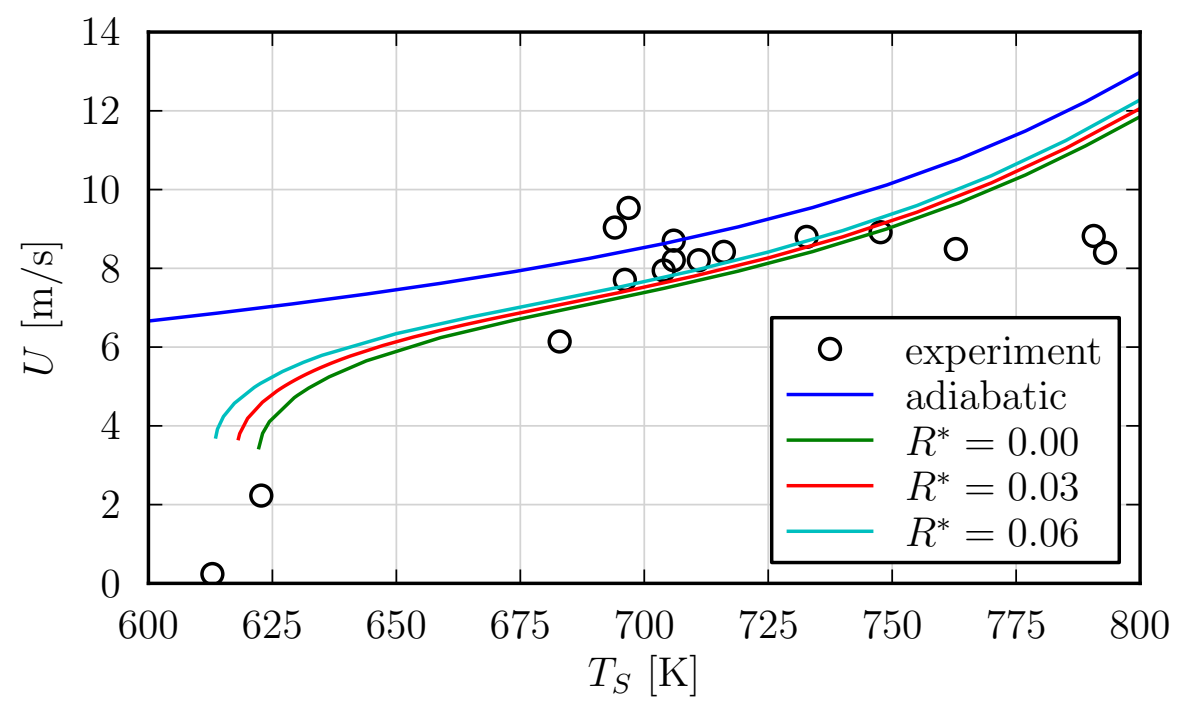

Figure 11.17.: Comparison of explosive crystallization wave propagation speed $U$ vs. $T_{S}$. Empty circles: experimental values from Grigoropoulos et al. (2006). Colored lines: computations for the adiabatic case and different values of $R^{*}$, using parameter set $\mathrm{C}$ and a kinetic prefactor $P=1500$.

A computation with $P=1$ does not yield results because the much larger value of $t_{C, \text { ref }}$ yields a value of $H$ which is larger than the critical value necessary for explosive crystallization in a wave of invariant temperature distribution.

\subsubsection{Effect of stopping crystallization below a certain temperature}

To be able to treat the present problem as a wave of invariant shape, it is necessary to stop the crystallization completely below a certain temperature greater than $T_{S}$. This cutoff temperature is, for simplicity, also designated as $T_{g}$ where no "real" glass transition temperature is contained in the crystallization kinetics, see (3.18). It is arbitrarily set as $T_{g}=T_{S}+10 \mathrm{~K}$ in the considered parameter sets, cf. Table 10.1. This approximation is normally well justified. This is because the exponential decrease of the crystallization rate with decreasing temperature - seen as an exponential increase in the crystallization time $t_{C} 4.8 \mathrm{~b}$ - means that crystallization just above $T_{g}$ is normally negligibly slow compared to the values at $T_{\text {ref }}$.

However, if $T_{S}$, and consequently $T_{g}$, becomes high enough, crystallization rate increases. Then it is possible that $1 / t_{C}$, as a measure of the crystallization rate, 


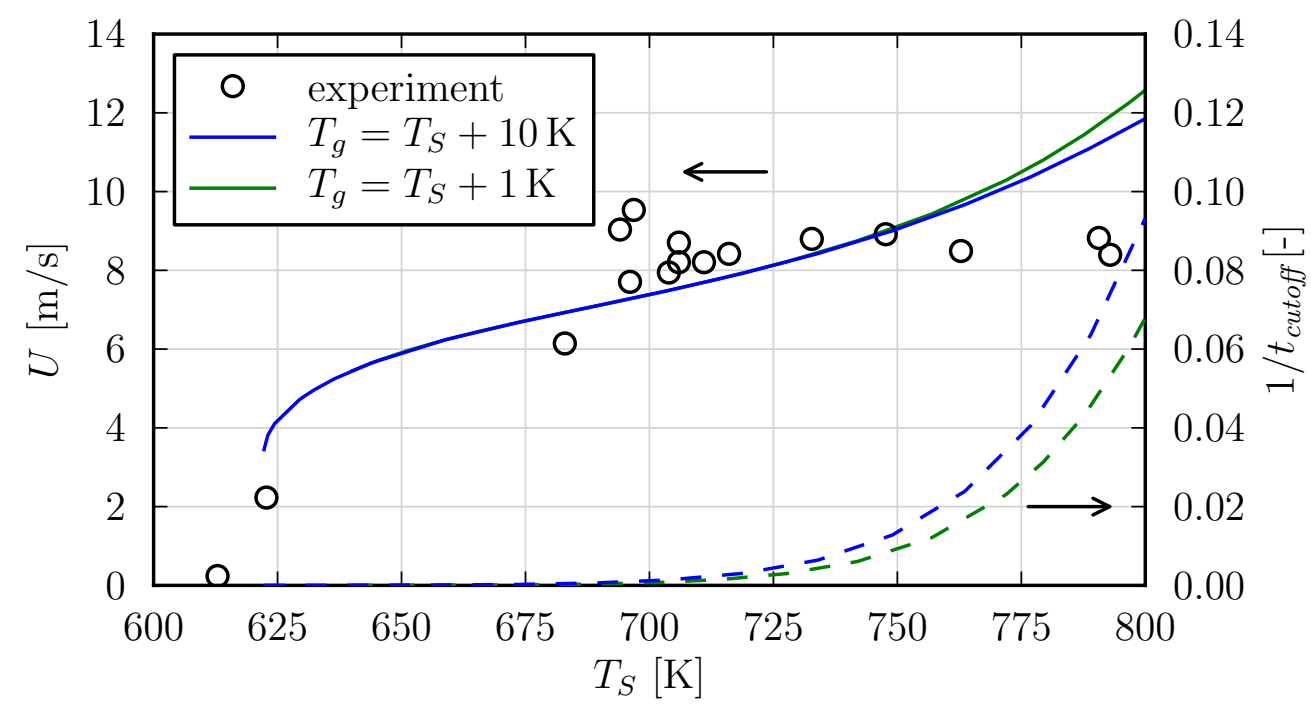

Figure 11.18.: Effect of choice of cutoff temperature $T_{g}$ : Empty circles indicate experimental values from Grigoropoulos et al. (2006). Solid lines indicate $U$ for the $R^{*}=0$ computation of fig. 11.17 (blue) and for a reduced offset for $T_{g}$ (green). Dashed lines show nondimensional $1 / t_{\text {cutoff }}$ according to (11.7).

becomes significant at $T_{g}$ with respect to its value at $T_{\text {ref }}$. This is also corroborated by reports in Grigoropoulos et al. (2006) that there is appreciable solid phase crystallization if the samples are kept at elevated temperatures for varying durations without initiating explosive crystallization. At this point it is convenient to define the nondimensional quantity

$$
t_{\text {cutoff }}:=\frac{\lim _{T \rightarrow T_{g}+} t_{C}(T)}{t_{C, \text { ref }}},
$$

which relates the crystallization times at $T_{g}$ and $T_{\text {ref }}$, respectively.

The effect of cutting off crystallization below $T_{g}$ on $U$ is shown in fig. 11.18; The only difference between the blue and green plots is a reduction in the glass transition temperature, from $T_{g}=T_{S}+10 \mathrm{~K}$ to $T_{g}=T_{S}+1 \mathrm{~K}$, respectively. On the left hand side, the values of $U$ for different offsets $T_{g}-T_{S}$ are identical, while on the right hand side they start to slowly diverge. However, the difference in $U$ remains below $10 \%$, indicating that the cutoff temperature approximation is reasonable for the $T_{S}$ values considered.

Values for $1 / t_{\text {cutoff }}$ are shown as dashed lines to illustrate the impact of the cutoff. On the left hand side, where the $U$ values are identical for both offsets, $1 / t_{\text {cutoff }}$ is 


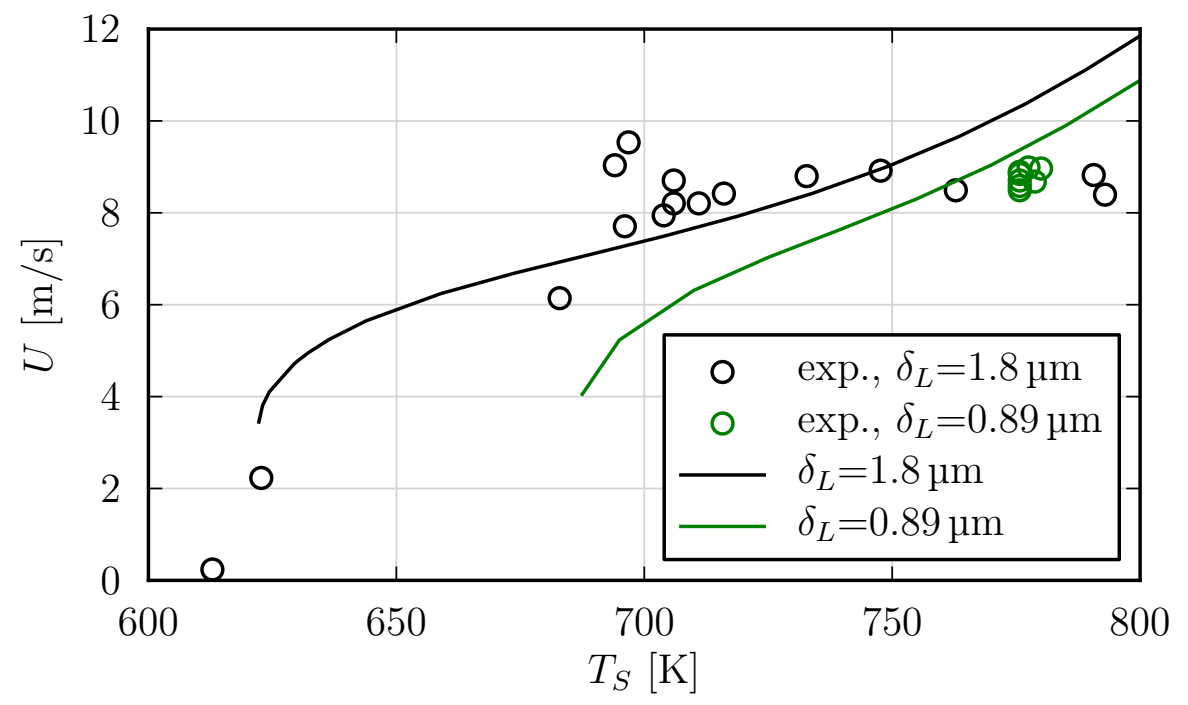

Figure 11.19.: $U$ vs. $T_{S}$ for two values of $\delta_{L}$, otherwise using parameter set $\mathrm{C}$, a kinetic prefactor $P=1500$ and $R^{*}=0$. Circles: experimental results from Grigoropoulos et al. (2006). Solid lines: computations.

very small, i.e. the crystallization rate is very small at $T_{g}$ compared to $T_{\text {ref }}$. On the right hand side, values of $1 / t_{\text {cutoff }}$ start to increase, but still stay small. The crystallization rate at $T_{g}$ stays below $10 \%$ of the value at $T_{\text {ref }}$.

Values of $H$ and $R^{*}$ have no influence on this analysis, as it is a purely a matter of crystallization kinetics. Results of adiabatic computations show identical behavior.

\subsubsection{Results for different layer thickness}

Grigoropoulos et al. (2006) also contains results for different layer thicknesses $\delta_{L}$. It is reported that a thinner sample with $\delta_{L}=0.89 \mu \mathrm{m}$ instead of $\delta_{L}=1.8 \mu \mathrm{m}$ would only explosively crystallize for $T_{S} \approx 750-800 \mathrm{~K}$, with a wave propagation speed $U$ identical to the thicker sample in that temperature region.

Figure 11.19 shows these experimental results, along with corresponding computations. It can be seen that for the two computations, the difference between the $U$ values at $T_{S} \approx 775 \mathrm{~K}$ is about as big as the variation in the experimental values, confirming the reported observation that $U$ is independent of $\delta_{L}$ in this temperature region.

Furthermore, for the thinner sample, explosive crystallization becomes impossible at a much higher $T_{S}$ than for the thicker sample. This is consistent with the experimentally observed limitation of explosive crystallization to higher substrate temper- 
atures for the thinner sample, even if the minimum value of $T_{S} \approx 750 \mathrm{~K}$ reported in Grigoropoulos et al. (2006) for explosive crystallization of the thinner sample is not reflected in the numerical results.

\subsubsection{Potential sources of discrepancies}

It was necessary to scale $G_{C}$ and $I_{C}$ with a relatively high prefactor of $P=1500$ to obtain values of $U$ comparable to the experimental results. However, it has to be noted that $P$ is only a multiplicative prefactor. Due to the exponential nature of (3.18) and (3.21), $G_{C}$ and $I_{C}$ are more sensitive to changes in the respective activation energies. Therefore, appropriate values of $U$ could also be reached with comparably small changes to the activation energies. However, this has not been done because there is a better agreement in the literature concerning the activation energies than concerning the multiplicative prefactors (Claverie et al., 2010).

The agreement between experimental results and the present model in figure 11.17 leaves room for improvement. For $T_{S}$ less than approximately $700 \mathrm{~K}$, the agreement seems reasonable.

Allowing for the influence of an unknown thermal contact resistance, the minimum $T_{S}$ for explosive crystallization in a wave of invariant shape to be possible agrees reasonably well with the experimentally observed minimum value for $T_{S}$. The value of $U$ at that $T_{S}$ is not accurate, though. It should be noted that the large variations of $U$ at a given $T_{S}$, e.g. at $T_{S} \approx 695 \mathrm{~K}$, and the small number of experimental data for low $T_{S}$, have to be taken into account when evaluating the agreement between model and experiment. The fact that $U \approx$ const for $T_{S} \gtrsim 700 \mathrm{~K}$ in the experimental results of Grigoropoulos et al. (2006) is not reflected in the results from the present model. The analysis in Köppl (1990) also shows a constant $U$ for supercritical crystallization waves. However, this is only exact in the asymptotic limit of large activation energies, which is not well-satisfied in the present problem.

Grigoropoulos et al. note that for $T_{S}$ less than approximately $700 \mathrm{~K}$, a direct amorphous-crystalline transition governs the observed explosive crystallization process, while for $T_{S} \gtrsim 700 \mathrm{~K}$, where $U \approx 8 \mathrm{~m} / \mathrm{s}$ stays constant, the process occurs with a thin layer of amorphous melt between the amorphous and crystalline phases. Ohdaira \& Matsumura (2012) report $U \approx 5-7 \mathrm{~m} / \mathrm{s}$, at unknown $T_{S}$, for mixed solidand liquid-phase explosive crystallization of germanium films with $\delta_{L} \approx 3 \mu \mathrm{m}$, using flash-lamp annealing. 
The theoretical model presented in Grigoropoulos et al. (2006) describes only the regime where $T_{S} \gtrsim 700 \mathrm{~K}$. Possibly the model presented here does not capture all necessary facets of the explosive crystallization process at $T_{S} \gtrsim 700 \mathrm{~K}$.

The one-dimensional approximation for the layer may not be well justified in regions where the thickness of the crystallization zone is not small compared to the layer thickness $\delta_{L}$, cf. fig. 11.15 .

\subsection{Future work}

The nature of the agreement between experiment and model indicates that there is room for improvement. Furthermore, there are still areas where the analysis could be extended. Implementing a crystallization model which includes the "liquid layer" often mentioned in the literature could possibly reproduce the region at high $T_{S}$ where $U$ is independent of $T_{S}$. Also, if data for $n_{C}$ can be obtained, heterogeneous crystallization could be considered instead of homogeneous crystallization. The influence of non-constant material parameters, varying with degree of crystallization or temperature (e.g. thermal diffusivity or density), could be examined. Computations for a transient process, while computationally more demanding, will be able to reproduce more features of the explosive crystallization process, e.g. the initiation process, or the dying out of crystallization waves due to large heat loss. Furthermore, a model for a transient process could be used to investigate the case of explosive crystallization ignited by point sources, which has not been considered in the present work. Further analytical investigations could shed more light on the exponential behaviour of $\lambda$ vs. $H \lambda$, on the dependence of $H_{\text {crit }}$ on various parameters, or on the linear dependence of $\lambda$ on $R^{*}$. Considering effects of instability on the crystallization front could yield different surface morphologies, as observed in the literature, see e.g. Frankel et al. (2000); Geiler \& Heinig (1985); Kurtze et al. (1984); Provatas et al. (1996); van Saarloos \& Weeks (1983); Shklovskij \& Ostroushko (1996); Smagin \& Nepomnyashchy (2009). Additional experiments could yield more data to validate the model against. Experiments should include measurements of $U$ and determine the crystallization mechanism involved. The comparably small values of $U$ for explosive crystallization in polymers (Köppl, 1990) could be favorable to experimental observation of the explosive crystallization wave. However, ensuring that $a \ll 1$ will be more challenging for polymers than for, e.g. germanium. As many of the param- 
eters relevant for the presented model as possible should be determined to reduce inaccuracies due to collecting parameters from different sources. 


\section{Summary}

In the present work, a model for explosive crystallization in a thin amorphous layer on a heat conducting substrate was developed. Up to four rate equations were used to describe the kinetics of the homogeneous amorphous-crystalline transition. For the thin layer the energy equation was used in a one-dimensional approximation. The whole process was described as a wave of invariant shape in a moving frame of reference. The propagation velocity of the wave $U$ was obtained as an eigenvalue of the system of equations.

The heat loss into the substrate was derived from first principles, with and without a thermal contact resistance at the interface between layer and substrate. This was done to arrive at a more precise description than a semi-empirical heat transfer coefficient, which is often used in the literature as a simple approximation of the heat loss. The temperature distribution in the substrate was found as a solution of the heat diffusion equation and was described in terms of a distribution of heat fluxes at the layer-substrate interface. It was assumed that the substrate's thermal diffusivity is much smaller than the thermal diffusivity of the layer. It was found that there is a non-local influence of the temperature distribution in the layer on the heat loss. As a result, the energy equation for the thin layer became an integro-differential equation.

A coupled system of one integro-differential equation and one to four ordinary differential equations was solved numerically. A precursor region was identified, where the system of equations could be solved analytically. A stretched coordinate system was used to map the semi-infinite range of the space coordinate $\eta$ in the moving frame of reference to a finite range in $z$. Using the stretched coordinate system introduced a singularity at $z=1$. Far behind the wave, the asymptotic behaviour of the temperature distribution was determined and used as a boundary condition near $z=1$ to avoid the singularity. The system of equations was solved using MATLAB.

Data for 19 different experimental and material parameters for explosive crystallization of germanium films on a quartz substrate were collated from a number of 
sources. Where no data were available, values were estimated from data for similar materials.

Some representative solutions of the system of equations were shown, demonstrating the key features of the process. The crystallization zone is short compared to the thermal preheating zone in front of the wave. When heat loss is considered, the cooling zone behind the wave is long compared to the pre-heating zone.

Systematically varying the enthalpy of fusion revealed more details. In the adiabatic case, a longer crystallization zone appears as the adiabatic end temperature approaches the melting temperature $T_{m}$. This is due to slow crystallization near $T_{m}$. Even incomplete crystallization is possible in the adiabatic case. When including heat loss, a plateau region of constant temperature can appear. In this region, heat loss into the substrate and liberation of latent heat due to slow crystallization are in balance until the amorphous phase is fully crystallized. Furthermore, the non-local influence of the heat loss is apparent in the plateau region.

Varying the non-dimensional heat loss parameter $H$, a critical value was found beyond which no crystallization wave of invariant shape is possible. This can also be interpreted as a certain minimum layer thickness. Additionally, a classification scheme suitable for explosive crystallization waves including heat loss was developed.

The length of the crystallization zone when varying $T_{S}$ was examined. Furthermore, two approximations, for large and small thermal contact resistance, respectively, were used to examine the influence of the thermal contact resistance on $U$.

Finally, crystallization wave speeds were compared with experimental values for explosive crystallization in germanium. It was necessary to adjust kinetic parameters to achieve a correct magnitude of $U$.

For $T_{S}$ up to about $700 \mathrm{~K}$, the agreement between model and experimental values is reasonable. The minimum $T_{S}$ for explosive crystallization in a wave of invariant shape to be possible agrees reasonably well with the experimentally observed minimum value for $T_{S}$. The value of $U$ at that $T_{S}$ is not accurate, though.

For $T_{S}$ larger than about $700 \mathrm{~K}$, the value of $U$ in the experiment remains approximately constant. This is not reflected in the model results. In the experiment, this region exhibits a different crystallization mechanism than for lower $T_{S}$. This mechanism is not captured in the model, which may account for the discrepancy.

The effects of the kinetic cutoff approximation, which enables treatment as a wave of invariant shape, were investigated. It was confirmed that the approximation is reasonable for the $T_{S}$ values considered. 
In the experiment, $U$ is nearly independent of the layer thickness at $T_{S} \approx 775 \mathrm{~K}$. This is in accord with the model.

Possible sources for the discrepancies between the experimental results and the model were identified and potential areas for future work were discussed. 


\section{A. Appendix}

In this appendix, material presented in the main body of this thesis is supplemented with additional information useful to the interested reader. Quantities introduced in the appendix are not reflected in the nomenclature.

\section{A.1. Derivation of rate equations for homogeneous crystallization}

The derivation of the rate equations for homogeneous nucleation is very similar to the derivation for heterogeneous crystallization, as given in Schneider et al. (1988). Instead of a probability of formation of growth nuclei from existing dormant nuclei $n_{C}$, a spontaneous crystal nucleation rate $I_{C}$ (see section 3.4 ) is used. Without taking crystal impingement, i.e. collisions between growing crystals, into account, the number of crystal nuclei per unit volume $N$ changes over time according to the relation

$$
\frac{\partial N}{\partial t}=I_{C} ; N(0)=\bar{N}=0
$$

For heterogenous crystallization, one has to distinguish between the number of existing nuclei and the number of "activated" (i.e. growing) nuclei. This distinction is not necessary for homogenous crystallization.

The volume of a single crystal growing from time $t^{\prime}=z$ to $t^{\prime}=t$, assuming it does not encounter another crystal, is

$$
v(t, z)=\sigma\left[\int_{z}^{t} G_{C} \mathrm{~d} t^{\prime}\right]^{m},
$$


if the volume of the crystal at $t=z$ is neglected. Thus, a "virtual" volume fraction of the crystalline phase without regarding impingement called the "extended" volume fraction $V_{e}$ is

$$
V_{e}(t)=\int_{0}^{t} v(t, z) \mathrm{d} N(z)=\int_{0}^{t} v(t, z) I_{C}(z) \mathrm{d} z .
$$

Following Avrami's model of impingement, with Mandelkern's correction for incomplete crystallization, as outlined in Schneider et al. (1988), it can be assumed that the rate of change of the crystalline volume fraction $V$ is proportional to the untransformed volume fraction $(1-\xi)$. Therefore, $\mathrm{d} V=(1-\xi) \mathrm{d} V_{e}$, which can be combined with A.3 and with the definition of the degree of crystallization (2.1) as follows:

$$
\begin{aligned}
\frac{\partial V}{\mathrm{~d} z} & =(1-\xi) \frac{\mathrm{d} V_{e}}{\mathrm{~d} z}=(1-\xi) v(t, z) I_{C}(z) \\
\frac{\mathrm{d} \xi}{\mathrm{d} z} & =\frac{1-\xi}{V_{\infty}} v(t, z) I_{C}(z) \\
-\ln (1-\xi) & =\frac{1}{V_{\infty}} \int_{0}^{t} v(t, z) I_{C}(z) \mathrm{d} z .
\end{aligned}
$$

Using the definition of $v(t, z)$, eq. A.2, this becomes an explicit double-integral expression for $\xi$. With 3.13 , it can be written as

$$
\phi_{C, 0}=\frac{\sigma}{V_{\infty}} \int_{0}^{t}\left[\int_{z}^{t} G_{C}\left(t^{\prime}\right) \mathrm{d} t^{\prime}\right]^{m} I_{C}(z) \mathrm{d} z
$$

After a differentiation with respect to time $t$, this becomes

$$
\frac{\partial \phi_{C, 0}}{\partial t}=m \frac{\sigma}{V_{\infty}} G_{C}(t) \int_{0}^{t}\left[\int_{z}^{t} G_{C}\left(t^{\prime}\right) \mathrm{d} t^{\prime}\right]^{m-1} I_{C}(z) \mathrm{d} z .
$$

Helper quantities $\phi_{C, i}$ can be defined as

$$
\phi_{i+1}=\frac{1}{G_{C}} \frac{\partial \phi_{C, i}}{\partial t} \quad(i=0 \ldots m-1)
$$

and (A.8) can be rewritten as

$$
\phi_{C, 1}=\frac{m \sigma}{V_{\infty}} \int_{0}^{t}\left[\int_{z}^{t} G\left(t^{\prime}\right) \mathrm{d} t^{\prime}\right]^{m-1} I_{C}(z) \mathrm{d} z .
$$


This equation can again be differentiated with respect to time. Using A.9), this becomes

$$
\phi_{C, 2}=\frac{m(m-1) \sigma}{V_{\infty}} \int_{0}^{t}\left[\int_{z}^{t} G\left(t^{\prime}\right) \mathrm{d} t^{\prime}\right]^{m-2} I_{C}(z) \mathrm{d} z .
$$

Another differentiation, with subsequent use of A.9 yields

$$
\phi_{C, 3}=\frac{m(m-1)(m-2) \sigma}{V_{\infty}} \int_{0}^{t} I_{C}(z) \mathrm{d} z .
$$

A.12 finally becomes, with $m=3$,

$$
\frac{\partial \phi_{C, 3}}{\partial t}=\frac{6 \sigma}{V_{\infty}} I_{C}(t)
$$

In contrast to heterogeneous crystallization and $L_{C}$, no characteristic length scale for crystallization is available for homogeneous crystallization. As a consequence, the quantities $\phi_{C, i}(i=1 \ldots m)$ are not dimensionless, but have the dimension Length $^{-\mathrm{i}}$.

The system of rate equation now finally becomes, using the impingement model of Avrami and three-dimensional crystal growth,

$$
\begin{aligned}
& \frac{\partial \phi_{C, i}}{\partial t}=G_{C} \phi_{C, i+1} \quad(i=0,1,2) \\
& \frac{\partial \phi_{C, 3}}{\partial t}=\frac{6 \sigma}{V_{\infty}} I_{C}
\end{aligned}
$$

This set of equations is repeated in the main body as (3.14). The relationship between $\phi_{C, 0}$ and $\xi$ is given by 3.13 . 


\section{Bibliography}

Andrä, G., Bergmann, J., Falk, F. \& Ose, E. 1998 Laser induced crystallization of amorphous silicon films on glass for thin film solar cells. physica status solidi (a) 166 (2), 629-634.

Andrä, G., Geiler, H. D., Götz, G., Heinig, K. H. \& Woittennek, H. 1982 Explosive liquid-phase crystallization of thin silicon films during pulse heating. physica status solidi (a) $\mathbf{7 4}$ (2), 511-515.

Avrami, M. 1939 Kinetics of phase change I. Journal of Chemical Physics 7 (12), $1103-1112$.

Avrami, M. 1940 Kinetics of phase change II. Journal of Chemical Physics 8 (2), 212-224.

Avrami, M. 1941 Kinetics of phase change III. Journal of Chemical Physics 9 (2), $177-184$.

Berger, J. 1988 Kristallisation von Kunststoffen unter dem Einfluss von Wärmeleitung und Kristallisationskinetik. PhD thesis, Vienna University of Technology.

Berthier, L. \& Biroli, G. 2011 Theoretical perspective on the glass transition and amorphous materials. Reviews of Modern Physics 83 (2), 587-645.

Bostanjoglo, O. \& Schlotzhauer, G. 1981 Impulse stimulated crystallization of Sb films investigated by time resolved TEM. physica status solidi (a) 68 (2), $555-560$.

Buchner, C. \& Schneider, W. 2010 a Crystallization waves in thin amorphous layers on heat conducting substrates. Proceedings in Applied Mathematics and Mechanics 10 (1), 493-494. 
Buchner, C. \& Schneider, W. $2010 b$ Explosive crystallization in thin amorphous layers on heat conducting substrates. In 2010 14th International Heat Transfer Conference, Volume 3, pp. 275-284. Washington, D.C.: ASME.

Buchner, C. \& Schneider, W. 2010c Explosive Kristallisation in dünnen amorphen Schichten auf wärmeleitenden Substraten. Poster, Jahrestreffen der ProcessNet-Fachausschüsse Computational Fluid Dynamics und Wärme- und Stoffübertragung.

Burger, M., Capasso, V. \& Eder, G. 2002 Modelling of polymer crystallization in temperature fields. Zeitschrift für Angewandte Mathematik und Mechanik 82 (1), 51-63.

Burger, M., Capasso, V. \& Micheletti, A. 2004 Optimal control of polymer morphologies. Journal of Engineering Mathematics 49 (4), 339-358.

Claverie, A., Koffel, S., Cherkashin, N., Benassayag, G. \& Scheiblin, P. 2010 Amorphization, recrystallization and end of range defects in germanium. Thin Solid Films 518 (9), 2307-2313.

Clevenger, L. A., Thompson, C. V. \& Tu, K. 1990 Explosive silicidation in nickel/amorphous-silicon multilayer thin films. Journal of Applied Physics 67 (6), $2894-2898$.

Coffin, C. C. 1935 a Studies on explosive antimony II: Its structure, electrical conductivity, and rate of crystallization. Royal Society of London Proceedings Series A 152 (875), 47-63.

Coffin, C. C. $1935 b$ Studies on explosive antimony III: The magnetic susceptibility. Canadian Journal of Research 13a (6), 120-125.

Coffin, C. C. \& Hubley, C. E. 1950 Studies on explosive antimony IV: The heat of explosion at $40^{\circ} \mathrm{C}$. Canadian Journal of Research 28b (10), 644-647.

Coffin, C. C. \& Johnston, S. 1934 Studies on explosive antimony I: The microscopy of polished surfaces. Proceedings of the Royal Society A: Mathematical, Physical and Engineering Sciences 146 (858), 564-570.

De Santis, F., Lamberti, G., Peters, G. W. M. \& Brucato, V. 2005 Improved experimental characterization of crystallization kinetics. European Polymer Journal 41 (10), 2297-2302. 
Donovan, E. P., Spaepen, F., Poate, J. M. \& Jacobson, D. C. 1989 Homogeneous and interfacial heat releases in amorphous silicon. Applied Physics Letters 55 (15), 1516-1518.

Donovan, E. P., Spaepen, F., Turnbull, D., Poate, J. M. \& Jacobson, D. C. 1985 Calorimetric studies of crystallization and relaxation of amorphous $\mathrm{Si}$ and Ge prepared by ion implantation. Journal of Applied Physics 57 (6), 1795.

Donovan, E. P., Spaepen, F., Umemoto, S., Poate, J. M. \& Jacobson, D. C. 1983 Heat of crystallization and melting point of amorphous silicon. Applied Physics Letters 42 (8), 698-700.

EDER, G. 1997 Fundamentals of structure formation in crystallizing polymers. In Macromolecular Design of Polymeric Materials (eds. K. Hatada, T. Kitayama \& O. Vogl), pp. 761-782. CRC Press.

Eder, G. \& Janeschitz-Kriegl, H. 1997 Crystallization. In Processing of Polymers (ed. H. E. H. Meijer), Materials Science and Technology, vol. 18, pp. 270-342. Weinheim: VCH.

Endo, Y., Fujiwara, T., Ohdaira, K., Nishizaki, S. \& Nishioka, K. 2010 Thin-film polycrystalline silicon solar cells formed by flash lamp annealing of a-Si films. Thin Solid Films 518 (17), 5003-5006.

FAN, J. C. C. 1981 Transition temperatures and heats of crystallization of amorphous $\mathrm{Ge}, \mathrm{Si}$, and $\mathrm{Ge}_{1-x} \mathrm{Si}_{x}$ alloys determined by scanning calorimetry. Journal of Applied Physics 52 (6), 4003-4006.

FArJas, J. \& Roura, P. 2006 Modification of the Kolmogorov-Johnson-MehlAvrami rate equation for non-isothermal experiments and its analytical solution. Acta Materialia 54 (20), 5573-5579.

Farjas, J. \& Roura, P. 2008 Simple approximate analytical solution for nonisothermal single-step transformations : Kinetic analysis. AIChE Journal 54 (8), $2145-2154$.

Floro, J. A. 1986 Propagation of explosive crystallization in thin Rh-Si multilayer films. Journal of Vacuum Science \& Technology A: Vacuum, Surfaces, and Films $4(3), 631-636$. 
Frankel, M., Gross, L. K. \& Roytburd, V. 2000 Thermo-kinetically controlled pattern selection. Interfaces and Free Boundaries 2 (3), 313-330.

Gall, S., Becker, C., Lee, K., Sontheimer, T. \& Rech, B. 2010 Growth of polycrystalline silicon on glass for thin-film solar cells. Journal of Crystal Growth 312 (8), 1277-1281.

Geiler, H. D., Glaser, E., Götz, G. \& Wagner, M. 1986 Explosive crystallization in silicon. Journal of Applied Physics 59 (9), 3091-3099.

Geiler, H. D. \& Heinig, K. H. 1985 Theoretical description of explosive crystallization phenomena induced by high temperature pulses in thin layers of amorphous semiconductors. In Energy Pulse Modification of Semiconductors and Related Materials, pp. 451-458. Dresden: Zentralinstitut für Kernforschung Rossendorf.

Gilmer, G. H. \& Leamey, H. J. 1980 An analysis of the explosive crystallization of amorphous layers. In Laser and Electron Beam Processing of Materials (eds. C. S. White \& P. S. Peercy), pp. 227-233. New York: Academic Press.

Gore, G. 1855 On a peculiar phænomenon in the electro-deposition of antimony. Philosophical Magazine Series 49 (56), 73-74.

Göтz, G. 1986 Explosive crystallization processes in silicon. Applied Physics A Solids and Surfaces 40 (1), 29-36.

GöTzBERGER, A. 1955 Über die Kristallisation aufgedampfter Antimonschichten. Zeitschrift für Physik 142 (2), 182-200.

Grigoropoulos, C., Rogers, M., Ko, S. H., Golovin, A. A. \& Matkowsky, B. J. 2006 Explosive crystallization in the presence of melting. Physical Review B 73 (18), 184125.

Grigull, U. \& Sandner, H. 1984 Heat conduction. International Series in Heat and Mass Transfer. Berlin: Springer.

Heinig, K. H. \& Geiler, H. D. 1985 Phenomenological theory of explosive solid phase crystallization of amorphous silicon I: Stationary solutions. physica status solidi (a) 92 (2), 421-430. 
Heinig, K. H. \& Geiler, H. D. 1986 Phenomenological theory of explosive solid phase crystallization of amorphous silicon II: Dynamical processes. physica status solidi (a) 93 (1), 99-104.

Hoffman, J. D. 1964 Theoretical aspects of polymer crystallization with chain folds: Bulk polymers. Polymer Engineering and Science 4 (4), 315-362.

Holman, J. P. 2010 Heat Transfer, 10th Ed. New York: McGraw-Hill.

HütTER, M. 2001 Thermodynamically consistent incorporation of the Schneider rate equations into two-phase models. Physical Review E 64 (1), 011209.

HütTeR, M. 2004 Crystallization under external pressure. Journal of NonNewtonian Fluid Mechanics 120 (1-3), 55-68.

Janeschitz-Kriegl, H. 2010 Crystallization Modalities in Polymer Melt Processing. Wien/New York: Springer.

Johnson, B., Gortmaker, P. \& McCallum, J. 2008 Intrinsic and dopantenhanced solid-phase epitaxy in amorphous germanium. Physical Review $B$ 77 (21), 214109.

Kitzhofer, G., Pulverer, G., Simon, C., Koch, O. \& Weinmüller, E. B. 2010 The new MATLAB code BVPSUITE for the solution of singular implicit BVPs. Journal of Numerical Analysis, Industrial and Applied Mathematics 1 (1), $1-23$.

KLuwiCK, A. 2008 Wellen in Flüssigkeiten und Gasen. Course at Vienna University of Technology.

Knapp, J. A. \& Picraux, S. T. 1981 Microsecond time-scale Si regrowth using a line-source electron beam. Applied Physics Letters 38 (11), 873-875.

Koba, R. \& Wickersham, C. E. 1982 Temperature and thickness effects on the explosive crystallization of amorphous germanium films. Applied Physics Letters 40 (8), 672-675.

Koba, R. \& Wickersham, C. E. 1983 Erratum: Temperature and thickness effects on the explosive crystallization of amorphous germanium films [Appl. Phys. Lett. 40, 672 (1982)]. Applied Physics Letters 42 (4), 398. 
Kokorowski, S. A., Olson, G. L. \& Hess, L. D. $1982 a$ Kinetics of laser-induced solid phase epitaxy in amorphous silicon films. Journal of Applied Physics 53 (2), 921-926.

Kokorowski, S. A., Olson, G. L., Roth, J. A. \& Hess, L. D. $1982 b$ Investigation of the melting temperature of amorphous silicon. Physical Review Letters 48 (7), 498-501.

Kolmogorov, A. N. 1937 On the statistical theory of the crystallization of metals (in Russian). Izv. Acad. Nauk. SSSR, Ser. Mat. 1 (3), 355-359.

KöPPL, A. 1990 Anwendung von Ratengleichungen auf anisotherme Kristallisation von Kunststoffen. PhD thesis, Vienna University of Technology.

KÖster, U. \& Herold, U. 1981 Crystallization of metallic glasses. In Glassy Metals I (eds. H.-J. Güntherodt \& H. Beck), Topics in Applied Physics, vol. 46, pp. 225-259. Berlin/Heidelberg: Springer.

van Krevelen, D. 1990 Properties of Polymers, 3rd Ed. Amsterdam: Elsevier Science.

Kumomi, H. \& Yonehara, T. 1994 Transient nucleation and manipulation of nucleation sites in solid-state crystallization of a-Si films. Journal of Applied Physics 75 (6), 2884-2901.

KuO, C.-C. 2009 Observation of explosive crystallization during excimer laser annealing using in situ time-resolved optical reflection and transmission measurements. Journal of Materials Processing Technology 209 (6), 2978-2985.

Kurtze, D. A. 1986 Stability of incomplete explosive crystal growth. Physical Review B 34 (3), 1770-1774.

Kurtze, D. A., van Saarloos, W. \& Weeks, J. D. 1984 Front propagation in self-sustained and laser-driven explosive crystal growth: Stability analysis and morphological aspects. Physical Review B 30 (3), 1398-1415.

Kuz'menko, V. M., Shklovskij, V. A. \& Vladychkin, A. N. 1991 Effect of crystalline inclusions on the explosive crystallization of amorphous films of pure metals. Journal of Non-Crystalline Solids 130 (3), 319-321. 
Linstrom, P. J. \& Mallard, W. G., eds. 2005 NIST Chemistry WebBook, NIST Standard Reference Database Number 69. Gaithersburg, MD: National Institute of Standards and Technology.

Ma, E., Thompson, C. \& Clevenger, L. A. 1990 Self-propagating explosive reactions in $\mathrm{Al} / \mathrm{Ni}$ multilayer thin films. Applied Physics Letters 57 (12), 12621264 .

MandelKeRn, L. 1964 Crystallization of polymers. New York: McGraw-Hill.

Marfaing, J. \& Marine, W. 1995 The amorphous to crystalline phase transition and vice versa. In Diffusionless Phase Transitions in Oxides (ed. C. Boulesteix), Key Engineering Materials, vol. 101-102, pp. 393-432. Zürich: Trans Tech Publications.

Marine, W. \& Marfaing, J. 1991 Solid state nucleation rate of Si and Ge near melting point. Phase Transitions 31 (1), 299-308.

Martins, J. J. \& Cruz Pinto, J. J. C. 2000 Non-isothermal crystallisation kinetics with instantaneous nucleation. Polymer 41 (18), 6875-6884.

Martins, J. J. \& Cruz Pinto, J. J. C. 2002 Evaluation of the instantaneous nucleation density in the isothermal crystallization of polymers. Polymer 43 (14), 3999-4010.

Nikolova, L., LaGrange, T., Reed, B. W., Stern, M. J., Browning, N. D., Campbell, G. H., Kieffer, J.-C., Siwick, B. J. \& Rosei, F. 2010 Nanocrystallization of amorphous germanium films observed with nanosecond temporal resolution. Applied Physics Letters 97 (20), 203102.

Ohdaira, K., Fujiwara, T., Endo, Y., Nishizaki, S. \& Matsumura, H. 2009 Explosive crystallization of amorphous silicon films by flash lamp annealing. Journal of Applied Physics 106 (4), 044907.

Ohdaira, K., Ishiı, S., Tomura, N. \& Matsumura, H. 2011 Microstructure of polycrystalline silicon films formed through explosive crystallization induced by flash lamp annealing. Japanese Journal of Applied Physics 50 (4), 04 DP01.

Ohdaira, K. \& Matsumura, H. 2012 Flash-lamp-induced explosive crystallization of amorphous germanium films leaving behind periodic microstructures. Thin Solid Films 524, 161-165. 
Ohdaira, K., Nishikawa, T. \& Matsumura, H. 2010a Variation of crystallization mechanisms in flash-lamp-irradiated amorphous silicon films. Journal of Crystal Growth 312 (19), 2834-2839.

Ohdaira, K., Nishikawa, T., Shiba, K., Takemoto, H. \& Matsumura, H. $2010 b$ Polycrystalline Si films with unique microstructures formed from amorphous Si films by non-thermal equilibrium flash lamp annealing. physica status solidi (c) 7 (3-4), 604-607.

Okuda, M., InABa, H. \& Usuda, S. 2003 Explosive crystallization mechanism in Sb-rich eutectic materials of phase change optical memory. In Proceedings of the European Phase Change and Ovonics Science Symposium, pp. 79-96.

Olson, G. L. \& Roth, J. A. 1988 Kinetics of solid phase crystallization in amorphous silicon. Materials Science Reports 3 (1), 1-77.

Olver, F., Lozier, D., Boisvert, R. \& Clark, C., eds. 2010 NIST Handbook of Mathematical Functions. Cambridge: Cambridge University Press.

PoAte, J. M. 1983 Some thermodynamic properties of amorphous Si. Nuclear Instruments and Methods in Physics Research 209-210 (1), 211-217.

Polman, A., Mous, D. J. W., Stolk, P. A., Sinke, W. C., Bulle-Lieuwma, C. W. T. \& Vandenhoudt, D. E. W. 1989 Epitaxial explosive crystallization of amorphous silicon. Applied Physics Letters 55 (11), 1097-1099.

Polman, A., Stolk, P. A., Mous, D. J. W., Sinke, W. C., Bulle-Lieuwma, C. W. T. \& Vandenhoudt, D. E. W. 1990 Pulsed-laser crystallization of amorphous silicon layers buried in a crystalline matrix. Journal of Applied Physics 67 (9), 4024-4035.

Polyanin, A. D. \& Manzhirov, A. V. 1999 Handbuch der Integralgleichungen: exakte Lösungen. Heidelberg: Spektrum Akademischer Verlag.

Provatas, N., Grant, M. \& Elder, K. R. 1996 Phase-field model for activated reaction fronts. Physical Review B 53 (10), 6263-6272.

Pumir, A. \& Barelko, V. 2001 Cold ignition of combustion-like waves of cryochemical reactions in solids. European Physical Journal B 22 (1), 71-77. 
Rogers, M., Ko, S. H. \& Grigoropoulos, C. 2006 In situ crystal growth during explosive crystallization. In Photon Processing in Microelectronics and Photonics $V$ (eds. T. Okada, C. B. Arnold, M. Meunier, A. S. Holmes, D. B. Geohegan, F. Träger \& J. J. Dubowski), Proceedings of the International Society for Optical Engineering, vol. 6106, p. 610614. San Jose: SPIE.

Rohde, M. 1994 Photoacoustic characterization of thermal transport properties in thin films and microstructures. Thin Solid Films 238 (2), 199-206.

Roura, P. \& Farjas, J. 2009 Structural relaxation kinetics for first- and secondorder processes: Application to pure amorphous silicon. Acta Materialia 57 (7), 2098-2107.

Roura, P., Farjas, J. \& Roca i Cabarrocas, P. 2009 Characterization of amorphous and nanostructured Si films by differential scanning calorimetry. Thin Solid Films 517 (23), 6239-6242.

VAn SaArloos, W. \& Weeks, J. D. 1983 Surface undulations in explosive crystallization: A thermal instability. Physical Review Letters 51 (12), 1046-1049.

Scheichl, S., Braun, S. \& Kluwick, A. 2008 On a similarity solution in the theory of unsteady marginal separation. Acta Mechanica 201 (1-4), 153-170.

SchneIder, W. 1978 Mathematische Methoden der Strömungsmechanik. Braunschweig: Vieweg.

SCHNEIDER, W. 1996 Crystallization waves in glassy materials. In Symposium on Trends in Applications of Mathematics to Mechanics (STAMM 96). Warsaw: Institute of Fundamental Technological Research.

SCHNEIDER, W. 2010 The influence of substrates on the propagation speed of crystallization waves. Personal Communication.

Schneider, W., Berger, J. \& Köppl, A. 1992 Non-isothermal crystallization of polymers: Application of rate equations. In Proceedings of the First International Conference on Transport Phenomena in Processing (ed. S. I. Guceri), pp. 10431054. Honolulu: Technomic Publ. Co.

Schneider, W., Köppl, A. \& Berger, J. 1988 Non-isothermal crystallization of polymers - system of rate equations. International Polymer Processing II (3/4), $151-154$. 
Schoenfeld, O., Hempel, T. \& Bläsing, J. 1994 Investigation of the transition from amorphous to microcrystalline silicon. physica status solidi (a) 143 (2), 323331.

Shanks, H., Maycock, P., Sidles, P. \& Danielson, G. 1963 Thermal conductivity of silicon from 300 to $1400{ }^{\circ}$ K. Physical Review 130 (5), 1743-1748.

Sharma, R. K., Bansal, S. K., Nath, R., Mehra, R. M., Bahadur, K., Mall, R. P., Chaudhary, K. L. \& GarG, C. L. 1984 Electron beam induced explosive crystallization of unsupported amorphous germanium thin films. Journal of Applied Physics 55 (2), 387.

ShklovskiJ, V. A. \& Kuz'menko, V. M. 1989 Explosive crystallization of amorphous substances. Soviet Physics Uspekhi 32 (2), 163-180.

Shklovskij, V. A. \& Ostroushko, V. N. 1996 Nonlinear resonance study of the periodic motion of the explosive crystallization front in glasses. Physical Review $B$ 53 (6), 3095-3106.

Smagin, I. \& Nepomnyashchy, A. 2009 Stability analysis of explosive crystallization front in the ESPE mode. Physica D: Nonlinear Phenomena 238 (6), 706-723.

Smith, M., McMahon, R., Voelskow, M., Panknin, D. \& Skorupa, W. 2005 Modelling of flash-lamp-induced crystallization of amorphous silicon thin films on glass. Journal of Crystal Growth 285 (1-2), 249-260.

Sontheimer, T., Becker, C., Bloeck, U., Gall, S. \& Rech, B. 2009 Crystallization kinetics in electron-beam evaporated amorphous silicon on $\mathrm{ZnO}$ :Al-coated glass for thin film solar cells. Applied Physics Letters 95 (10), 101902.

Spaepen, F. \& Turnbull, D. 1982 Crystallization processes. In Laser Annealing of Semiconductors (eds. J. M. Poate \& J. W. Mayer), pp. 15-42. New York: Academic Press.

Spinella, C., Lombardo, S. \& Priolo, F. 1998 Crystal grain nucleation in amorphous silicon. Journal of Applied Physics 84 (10), 5383-5414.

Stolk, P. A., Polman, A. \& Sinke, W. C. 1993 Experimental test of kinetic theories for heterogeneous freezing in silicon. Physical Review B 47 (1), 5-13. 
Takamori, T., Messier, R. \& Roy, R. 1972 New noncrystalline germanium which crystallizes "explosively" at room temperature. Applied Physics Letters $20(5), 201$.

Thompson, M. O., Galvin, G. J., Mayer, J. W., Peercy, P. S., Poate, J. M., Jacobson, D. C., Cullis, A. G. \& Chew, N. G. 1984 Melting temperature and explosive crystallization of amorphous silicon during pulsed laser irradiation. Physical Review Letters 52 (26), 2360-2363.

Vega, F., Chaoui, N., Solis, J., Armengol, J. \& Afonso, C. N. 2005 Optical evidence for a self-propagating molten buried layer in germanium films upon nanosecond laser irradiation. Journal of Applied Physics 97 (10), 103519.

Voutsas, A. 2003 A new era of crystallization: Advances in polysilicon crystallization and crystal engineering. Applied Surface Science 208-209, 250-262.

Waheed, N. \& Rutledge, G. C. 2005 Crossover behavior in crystal growth rate from n-alkane to polyethylene. Journal of Polymer Science Part B: Polymer Physics 43 (18), 2468-2473.

Wickersham, C. E. 1983 Optical information storage using explosive crystallization in amorphous films. Journal of Vacuum Science \&5 Technology A: Vacuum, Surfaces, and Films 1 (4), 1857-1860.

Williams, M. L., Landel, R. F. \& FerRy, J. D. 1955 The temperature dependence of relaxation mechanisms in amorphous polymers and other glass-forming liquids. Journal of the American Chemical Society 77 (14), 3701-3707.

Zhao, Y., Zhu, C., Wang, S., Tian, J. Z., Yang, D. J., Chen, C. K., Cheng, H. \& Hing, P. 2004 Pulsed photothermal reflectance measurement of the thermal conductivity of sputtered aluminum nitride thin films. Journal of Applied Physics $96(8), 4563$.

Zuidema, H., Peters, G. W. M. \& Meijer, H. E. H. 2001 Influence of cooling rate on pVT data of semicrystalline polymers. Journal of Applied Polymer Science 82 (5), 1170-1186. 